Article

\title{
Emerging Biomarkers of Multiple Sclerosis in the Blood and the CSF: a Comprehensive Review of the Literature with a Focus on Neurofilaments and Therapeutic Considerations
}

\author{
Tamás Biernacki' ${ }^{1}$ Zsófia Kokas' ${ }^{1}$, Dániel Sandii ${ }^{1}$ Judit Füvesii ${ }^{1}$, Zsanett Fricska-Nagy ${ }^{1}$, Péter Faragó ${ }^{1}$, \\ Tamás Zsigmond Kincses ${ }^{1,2}$, Péter Klivényi ${ }^{1}$, Krisztina Bencsik ${ }^{1}$ and László Vécsei ${ }^{1,3}$

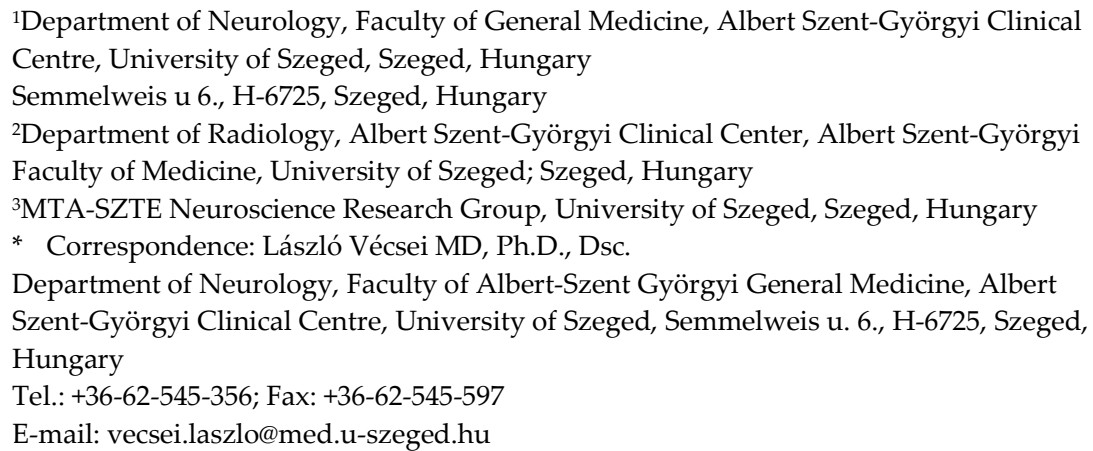

\begin{abstract}
Introduction: multiple sclerosis (MS) is the most common immunemediated chronic neurodegenerative disease of the central nervous system (CNS), affecting young people. Due to the permanent disability, cognitive impairment, and the enormous detrimental impact MS can exert on the patients' health-related quality of life. It is of great importance to recognise it in time and commence adequate treatment at an early stage. The currently used disease-modifying therapies (DMT) aim to reduce disease activity and thus halt disability development, which in current clinical practice are monitored by clinical and imaging parameters but not by biomarkers found in blood and/or the cerebrospinal fluid (CSF). Both clinical and radiological measures routinely used to monitor disease activity lack information on the fundamental pathophysiological features and mechanisms of MS. Furthermore, they lag behind the disease process itself. By the time a clinical relapse becomes evident or a new lesion appears on the MRI scan, potentially irreversible damage has already occurred in the CNS. In recent years several biomarkers that previously have been linked to other neurological and immunological diseases have received increased attention in MS. Additionally, other novel, potential biomarkers with prognostic and diagnostic properties have been detected in the CSF and blood of MS patients.
\end{abstract}

Areas covered: in this review, we summarise the most up to date knowledge and research conducted on the already known and most promising new biomarker candidates found in the CSF and blood of MS patients.

Author commentary: the current diagnostic criteria of MS rely on three pillars; MRI imaging, clinical events and the presence of oligoclonal bands in the CSF (which was reinstated into the diagnostic criteria by the most recent revision). Even though the most recent McDonald criteria made the diagnosis of MS faster than the prior iteration, it is still not an infallible diagnostic toolset, especially at the very early stage of clinically isolated syndrome. Together with the gold 
standard MRI and clinical measures, ancillary blood and CSF biomarkers may not just improve diagnostic accuracy and speed but very well may become agents to monitor therapeutic efficacy and make even more personalised treatment in MS a reality in the near future. The major disadvantage of these biomarkers in the past has been the need to obtain CSF to measure them. However, the recent advances in extremely sensitive immunoassays made their measurement possible from peripheral blood even when present only in minuscule concentrations. This should mark the beginning of a new biomarker research and utilisation era in MS.

Keywords: biomarker; diagnostic; prognostic; blood; cerebrospinal fluid; multiple sclerosis; disability progression

\section{Introduction}

Multiple Sclerosis (MS) is a common, autoimmune inflammatory and degenerative disease of the central nervous system (CNS) that results in demyelination and long term accumulation of disability. It is most prevalent in the northern hemisphere, affects women more than men, usually manifests in middle-aged patients. Interestingly, both the absolute prevalence and female dominance are reported to continuously increase in the past several decades ${ }^{1-3}$. Even though some symptoms frequently accompany MS, there are no pathognomic symptoms or clinical findings specific to it. Accordingly, MS can present with a wide variety of clinical and imaging changes $(4,5)$. Before the latest iteration of the McDonald criteria, studies reported that more than half of the patients diagnosed with clinically isolated syndrome (CIS) did not meet the diagnostic criteria for MS. However, after several years of followed-up, most of these patients eventually did convert to definite MS6,7. To overcome this sometimes several year-long lag in the diagnosis, an update was issued to the McDonald criteria in $2017^{8}$. The revision still considers MRI as the golden standard in the diagnosis of MS, however, the reintroduction of oligoclonal bands into the diagnostic toolset also made the diagnostic process easier and much faster. Unfortunately, MRI is not infallible either, as not all demyelinating changes are detected; up to $20 \%$ of CIS patients without any characteristic lesions will eventually convert to definite $\mathrm{MS}^{9-11}$. A growing body of research points towards the multifactorial cause of $\mathrm{MS}^{12}$. The exact pathogenesis, several mechanisms and the initiating step in the domino leading to MS are still unclear and opaque. However, the nowadays still unfolding interplay between genes and epigenetic regulatory mechanisms, viral ${ }^{13}$, environmental and lifestyle risk factors most certainly play a significant role ${ }^{12}$. The principle pathology of MS is characterised by the breakage of the blood-brain barrier, the subsequent infiltration of the CNS by autoreactive lymphocytes followed by demyelination, focal inflammation, eventually culminating in axonal loss, gliosis, white and grey matter atrophy ${ }^{12,14}$. There are three major clinical forms described; most ( $\sim 85 \%$ of all patients) present with relapsing-remitting multiple sclerosis (RRMS), which is characterised by periods of disease worsening (relapses) followed by complete or near-complete resolution of symptoms (remission) with or 
without persisting residual disability ${ }^{15,16}$. Without treatment after $15-20$ years, the reparative and regenerative capacity of the CNS depletes, and the cyclicity of relapses and remissions is superseded by the continuous accumulation of disability, with or without superimposed relapses, termed secondary progressive MS (SPMS) ${ }^{15,16}$. In a minority ( $15 \%)$ of MS patients, the disease follows a primary progressive (PPMS) course; from the beginning of the disease, continuous progression and disability accumulation are seen, essentially without any relapses or remissive phase ${ }^{15,16}$. In everyday practice, disease activity is monitored by the frequency of relapses (annual relapse rate - ARR), confirmed disability progression (CDP) - a significant increase of the expanded disability status scale [EDSS] ${ }^{17}$ that is sustained for at least 3 or 6 months ${ }^{18,19}$ and by various MRI parameters (e.g. new or unequivocally enlarging T2 lesions, contrast enhancement, grey matter atrophy) ${ }^{20}$. There are several shortcomings with these clinical measures. Due to the currently used disease-modifying drugs, the ARR of treated patients has fallen so drastically that it became an insensitive marker of disease activity. Also, the specificity and sensitivity of both CDP and ARR are contingent on the frequency of sampling, i.e. the interval between patient visits. Also, most of the accumulating disability in RRMS was shown to be independent of relapse activity, indicating a subtle, but continuously present progression ${ }^{21}$. Moreover, the EDSS score is not entirely objective; it is highly dependent on the rater shows great inter-rater and intra-rater variability. Furthermore, the patient's EDSS score may still fluctuate after six months, sometimes as much as 24 months of follow-up may be necessary for it to stabilize ${ }^{22,23}$. Another pitfall of the currently used disease activity monitoring methods is their temporal displacement. They are unable to forecast future activity by the time they signal the disease has already progressed (i.e. the damage resulting in the relapse has already occurred, the new lesion seen on the MRI represents inflammation already in progress). The same problem is encountered in everyday practice when a new patient presents with a short disease history. The currently used markers cannot predict future disease activity and the patient's disease course. Sometimes, it can be extremely challenging to accurately identify a specific disease subtype in the absence of a longer disease course.

In light of this, it is of no surprise that there is an enormous unmet medical need for diagnostic and prognostic biomarkers that can reliably predict a patient's disease subtype, disease activity and response to treatment before damage is suffered and permanent disability sets in. This might just become a reality soon as various biomarkers have been discovered in CSF and blood of MS patients linked to pathological processes thought to be involved in MS over the past several years.

Furthermore, the recent progress in analytical technologies made possible the accurate measurement of biomarkers present in the blood only in minuscule concentrations. This has allowed for less invasive and repeat sampling and has resulted in the surge of research that is seen nowadays, of which neurofilament light chain and the micro RNA assays are great examples. Nonetheless, compared with MRI and CSF oligoclonal 
bands (owing primarily to their lack of specificity and pending validation), these markers have limited contribution to MS diagnosis yet. Therefore are not currently included in the diagnostic criteria at this time. However, this might change shortly should the now seen speed and quality of research continue in the years to come.

\section{Glial fibrillary acidic protein (GFAP)}

GFAP is a monomeric, type III intermediate filament protein of 8-9 nm in length coded by the GFAP gene located on the long arm of chromosome 17 in humans ${ }^{24,25}$. It is expressed in various cell types in the body during development. Still, in the central nervous system, it is almost exclusively produced by and found in the cytoplasm of mature astrocytes. The exact role of the GFAP today is elusive, but it is primarily thought to play a role in the upkeep of the shape and provide mechanical strength for the astrocytes ${ }^{26}$. Elevated levels of GFAP can be detected in both the blood and CSF after the hyperplasia of the astrocyte population in the CNS. The human brain reacts with astrocytosis and glial scarring to different kinds of insults (trauma, chemical damage, various genetic and non-genetic based disorders, etc.), which results in elevated levels of GFAP released into the $\mathrm{CSF}^{27}$. Several studies ${ }^{28-36}$ have demonstrated significant differences in CSF GFAP levels between healthy controls and MS patients, also among different disease subtypes. A most recent meta-analysis ${ }^{37}$, has shown a mean difference in CSF GFAP levels of 0.62 (95\% CI=0.56-0.88, $\mathrm{p}<0.001)$ between the whole MS cohort and healthy controls (HC). A mean difference of 0.63 (95\% CI=0.39 to 0.86 ; $\mathrm{p}<0.001$ ) was observed between RRMS patients and HCs, meanwhile an enormous difference of 103.83 (95\% CI=68.09 to 139.57 ; $\mathrm{p}<0.001)$ was seen between RRMS patients in remission and during a relapse. The mean difference in CSF GFAP levels between progressive MS patients and HCs was 1.02 (95\% CI=0.73 to 1.31; $\mathrm{p}<0.001$ ), in contrast, progressive MS patients had lower CSF GFAP concentrations than RRMS patients did. $(\mathrm{SMD}=-0.47 ; 95 \% \mathrm{CI}=-0.80$ to $-0.15 ; \mathrm{p}=0.005)$. There was no difference in CSF GFAP levels between secondary and primary progressive patients (0.35, 95\% $\mathrm{CI}=-0.10$ to $0.79 ; \mathrm{p}=0.12)$. Interestingly neither natalizumab nor mitoxantrone or rituximab affected CSF GFAP levels ${ }^{38,39}$. Furthermore, CSF GFAP levels have shown a positive correlation with disease duration; this might be explained by the higher degree of astrogliosis accompanying disease progression ${ }^{40}$.

There are much fewer studies examining GFAP levels from the blood than research evaluating CSF GFAP levels. A study based on 245 MS patients and 53 controls demonstrated that MS patients had higher blood GFAP levels (difference 37.25, 95\% $\mathrm{CI}=21.3$ to $53.20 ; \mathrm{p}<0.001$ ) compared to HCs ${ }^{28,32,41}$. After differentiation by disease subtype, patients with relapsing-remitting disease were found to have similar blood GFAP levels to control subjects ( $\mathrm{SMD}=0.22 ; 95 \% \mathrm{CI}=-0.10$ to $0.54 ; \mathrm{p}=0.18$ ). In contrast to CSF GFAP levels, PPMS patients had higher serum GFAP concentrations than 
RRMS patients. Also, serum, but not CSF GFAP levels, correlated with disease severity, especially in patients with a primary progressive disease $28,32,41$.

CSF levels of GFAP seem to be correlated with MS and different disease subtypes, reflecting the different extent of damage to astrocytes and subsequent astrogliosis observed in different disease subtypes. As such, GFAP levels may help to differentiate PPMS and RRMS in their early stages, when it is not yet easy to discern the two from each other. Furthermore, GFAP might become a valuable marker of disease severity and progression. Nonetheless, more research with larger cohorts is needed to validate these findings, especially in the case of GFAP measures from the serum.

Leptin

Leptin is a peptide consisting of 167 amino acids, weighing $16 \mathrm{kDa}$. In the human body, it is mainly produced by white adipose tissue cells and enterocytes, T-lymphocytes and bone marrow cells $s^{42,43}$. It is encoded by the $\mathrm{Ob}$ gene on the long arm of chromosome $7^{44}$. Leptin exerts its effect through the leptin receptor (a member of the type I cytokine receptor family ${ }^{45}$ with a single transmembrane domain). It has a pivotal role in regulating several processes such as angiogenesis, wound healing, blood clotting, hunger, energy balance and expenditure, fat storage, hematopoiesis and last, but not at least, the immune and inflammatory response of the body ${ }^{46,47}$. In recent years variations in leptin levels were implicated in the development of MS and other autoimmune diseases ${ }^{48}$, as leptin was found to be a key modulator of the immune system $^{49}$. Leptin was shown to have an effect on the neutrophil and macrophage cell lines $^{50}$, promote autoreactive $\mathrm{T}$-cell proliferation, inhibit the proliferation of Tregcells $^{51}$, also to promote the secretion and phosphorylation of several proinflammatory cytokines (IL-2 and 6, Bcl-2, p-STAT3, TNF- $\alpha$, IFN- $\gamma$, pERK1/2, p27kip1) ${ }^{52}$. The altered expression of these cytokines can push the ratio of Th1 and Th2 regulatory T-cells out of balance, a process associated with the development of MS53-55.

The results of studies exploring circulating leptin levels in MS patients and its potential role as a biomarker and pathogenesis of MS are conflicting. The more significant part of the studies in the literature has found either elevated or no difference in the circulating leptin levels of MS patients compared to healthy controls. On the other hand, some persuasive papers found the exact opposite.

The largest meta-analysis to date in the literature assessing this matter evaluated nine studies, including 645 MS patients and 586 healthy control subjects. Despite a considerable heterogeneity among the articles on which the meta-analysis was based compared to controls, MS patients were found to have significantly higher circulating leptin levels (SMD $=0.70,95 \%$ CI 0.24 to $1.15, \mathrm{p}<0.001)^{42}$. These findings were backed by another study ${ }^{51}$, which has found, that being overweight at the age of 15 and being obese in young adulthood (at the age of 20) increases the risk of developing MS by 
more than twofold $(\mathrm{OR}=2.16, \mathrm{p}=0.01$ and $\mathrm{OR}=3.9, \mathrm{p}=0.01$, respectively). Similarly, compared to controls, higher leptin levels were seen in RRMS, and even higher levels were measured in SPMS patients. Another adipose tissue originating cytoplasmic protein, adipocyte fatty acid binding protein (A-FABP), has been markedly elevated in pediatric-onset MS patients compared to control subjects ${ }^{56}$. A Swedish biobank based study that examined the risk for developing MS based on circulating leptin and insulin levels in patients younger than 40 years corroborated these findings ${ }^{57}$. It has found sex and age-related correlation between leptin levels and the risk of developing MS. Higher leptin levels were associated with increased risk of MS in individuals (both men and women) younger than 20 years ( $\mathrm{OR}=1.4,95 \% \mathrm{CI}=1.1-1.9)$ and in all evaluated men $(\mathrm{OR}=1.4,95 \% \mathrm{CI}=1.0-2.0)$. In contrast, for women aged 30-39 years, there was a lower risk of MS with increased leptin levels (OR $=0.74,95 \% \mathrm{CI}=0.54-1.0)$. The majority of formerly published papers support the argument that elevated leptins are a risk factor for developing MS ${ }^{56,58-62}$. Conversely, a most recent study that has assessed different genetic polymorphisms in the leptin and leptin receptor gene has found contradicting results. This Kuwaiti based study ${ }^{63}$ (where obesity is prevalent in the general population) has measured lower leptin levels in MS patients compared to healthy controls. Additionally, they have found that a specific (rs7799039AA) leptin genotype is associated with an elevated risk of developing MS. However, it was found to bear no effect on the lower leptin levels observed.

Unfortunately, most of the studies mentioned above examining leptin's association with MS had several serious limitations. Many of the studies had small compound sample sizes, which in many cases diminished to be on the verge of losing statistical power after stratification into the subgroups of interest. Furthermore, most of the articles have failed to correct for several factors known to influence leptin levels (age, sex, smoking status, BMI, treatment status - both disease-modifying drug and steroid administration - of the MS population, disease subtype). Moreover, there is heterogeneity in the samples types that leptin was measured from (serum vs plasma, fasting vs non-fasting sampling). Due to these severe biases, the results and comparability of most of the studies are questionable, at least.

To conclude, there is significant controversy surrounding leptin in MS. Leptin's suspected contribution to the pathogenesis of MS is based on its ability to modulate the immune system by promoting the production of pro-inflammatory cytokines and recruiting immune cells. However, based on currently available data, there is no compelling evidence favouring leptin being a key player in MS pathogenesis, nor its use as a biomarker in MS. 


\section{Brain-derived neurotrophic factor}

The gene encoding brain-derived neurotrophic factor (BDNF) is located on the short arm of chromosome 11 comprises 11 exons and nine promoters. Due to different splicing, at least 34 different mRNA transcripts are produced in response to different stimuli ${ }^{64-66}$. BDNF is a member of the neurotrophin family, which consists of four major subtypes of structurally and functionally related proteins; BDNF, nerve growth factor (NGF), neurotrophin-3 (NT-3) and neurotrophin-4 (NT-4). These proteins share similar characteristics regarding their primary structure, molecular weight and function. BDNF is widely expressed in the central nervous system and is widely recognised as a major regulator protein for various types of neurons in both the adult and the developing brain ${ }^{64,67,68}$. BDNF plays a vital role in regulating several signalling pathways that control the survival, growth, differentiation and apoptosis of various cell types and is thus pivotal in neural development, neural plasticity and the longterm potentiation of synapses ${ }^{69-72}$. Two major receptors mediate its effects; the more sensitive TrkB and the low-affinity 775 receptors $^{73}$. A tremendous amount of attention and research regarding the genetic variations in the gene coding BDNF has been directed towards the single-nucleotide polymorphism (SNP) rs6265, which causes a valine to methionine substitution at codon 66 (Val66Met). This mutation alters the prodomain structure of the gene, which is functional; however, it leads to improper protein folding. This folding failure causes impaired and decreased activitydependent BDNF release, reduces BDNF binding to its TrkB receptor, and results in altered protein-protein interactions and conformational stability ${ }^{74}$. The Val66Met mutation does not show an even geographical distribution, as significant ethnic differences are reported in various studies. The Met carriers (Met/Met or Val/Met genotype) make up roughly $1 / 3^{\text {rd }}$ to half of the caucasian population across the USA and Europe. Meanwhile, the Met carrier frequency is much higher ( 70\%) in Asian populations (China, Japan and Korea) ${ }^{75-80}$. In recent years the Val66Met mutation has been linked to a plethora of psychiatric (depression, bipolar and anxiety disorders, eating disorder, PTSD, schizophrenia, etc.) and neurodegenerative (Huntington's disease, Parkinson's disease, Alzheimer's disease, ALS, MS) diseases. Other polymorphisms of the BDNF gene are also connected to neuronal disorders; the C270T polymorphism has been associated with late-onset Alzheimer's disease and MS ${ }^{81,82}$. Meanwhile, the rs2030324 mutation is linked to impaired visual cognitive processing among MS patients ${ }^{83}$. In contrast to these findings, several studies refute the associations above as they failed to find compelling evidence to link these alterations in the BDNF gene to either Alzheimer's disease or MS ${ }^{84-88}$. The variance in carrier prevalence across the globe maybe, at least, in part a possible explanation for the various contradicting associations demonstrated between these genetic polymorphisms and neuronal disorders. 
Similarly to other neurodegenerative diseases, the prognostic and diagnostic value of BDNF in MS is highly controversial. The Val66Met polymorphism has been linked to enhanced grey matter atrophy compared to Val/Val carriers in one study ${ }^{89}$, which was refuted by subsequent articles ${ }^{90,91}$. These findings are further shaded by an fMRI based study, which explored the Val66Met polymorphism's possible influence on the episodic memory of MS patients. It found that wild type Val/Val carrier RRMS patients compared to Met carrier patients showed greater brain responses during both encoding and retrieval trials on the episodic memory test administered. In contrast, the exact opposite was true for healthy controls. Conversely, a more robust hippocampus-posterior cingulate cortex connectivity was observed in Met carriers compared to Val homozygotes. The exact opposite was true for healthy controls, however ${ }^{92}$. On the other hand, a most recent study demonstrated that Met carrier status results in low BDNF expression and is a protective factor against cognitive impairment in MS. It has been found to be also associated with worse physical status and to be more prevalent in males ${ }^{93}$. Refuting both previously mentioned papers, a third paper has concluded that BDNF levels show no correlation with the presence of Met/Val polymorphism, or with the patients' physical status, or any of the psychometric tests used nor with any of the various MRI parameters measured in the study $^{94}$. To add more to the controversy in one cohort of MS patients, Val homozygoteousness was associated with younger disease onset in male patients. Meanwhile, it was linked to increased MS susceptibility in females implying a genderspecific effect of the polymorphism ${ }^{82}$. In opposition, several others have failed to show any impact of the BDNF Val66Met polymorphism on the susceptibility, severity, or clinical course of the disease ${ }^{88,95,96}$. As the sole evaluation of the presence or absence of the mutation has resulted in ambiguous results, it was theorised that not merely the polymorphism itself but its epigenetic regulation, namely the methylation status of the BDNF gene, may play a role in the expression and production of BDNF and thus the pathogenesis and progression of MS. A recent study ${ }^{97}$, based on a relatively large Italian cohort, assessed this hypothesis and found that merely the presence of rs6265 SNP itself was not a predictor of the severity of the disease. On the other hand, the critical role of epigenetic mechanisms was confirmed: a lower percentage of methylation of the BDNF gene was associated with a more severe and rapidly progressing disease. Given that higher methylation of a gene results in its silencing, these results suggest that a lower inhibition of the gene (i.e. hypomethylation) results in its hyperexpression (and therefore increased BDNF production in this case) is associated with a more severe disease course. Bearing in mind that BDNF is considered a neurotrophic factor, it is reasonable to presume that the level of methylation of the BDNF gene is a result of disease activity and not the other way around. It is plausible that patients with a more severe course and a higher level of inflammation resort to de-methylation as a defence mechanism, resulting in more increased secretion of 
BDNF, thus suppressing the ongoing inflammation and maintaining as many remaining neural functions as possible. On a similar train of thought, patients with a milder disease activity don't need to ease the methylation of the BDNF gene to subside inflammation in the CNS. Several neuropathological findings support this theory. Both BDNF and its receptor are readily expressed in the vicinity of and in the MS plaques themselves. Furthermore, „burnt out” older and chronic MS plaques seen in later stages of the disease were shown to contain a lesser amount of $\mathrm{BDNF}^{98}$, which may be one element responsible for the continuously ongoing axonal degeneration in the chronic progressive stage of MS ${ }^{99-101}$. Furthermore, evidence supports that neuronal BDNF might be pivotal to the endogenous neurotrophic repair following axonal damage seen in MS lesions ${ }^{102,103}$.

To add to the controversy, there is substantial inconsistency about the measured blood levels of BDNF in different stages and subtypes of MS. Some have found elevated BDNF in the sera of patients in the relapsing phase ${ }^{104,105}$. In contrast, others have found lower than normal serum levels of BDNF in RRMS patients, regardless of them being in remission or in relapse ${ }^{106}$. Similarly, when compared to HCs, all MS patients (regardless of subtype) on a population level had lower BDNF levels. The lowest concentrations were measured in SPMS patients, followed by RRMS patients. This is in line with prior findings that both serum and CSF levels of BDNF are reduced in MS patients compared to $\mathrm{HCs}^{107,108}$, and that BDNF levels are higher in RRMS than in progressive MS. This further supports the theory that progression in MS may be, in part, at least due to the exhaustion of the endogenous neuroprotective and reparative systems by the long-standing chronic inflammation ${ }^{99-101,105,108}$.

To summarise, BDNF levels and the presence or absence of the Val66Met polymorphism based on current evidence do not seem to be useful biomarkers for predicting disease susceptibility, progression, or identifying disease subtypes on a patient level. BDNF levels have been relatively concordantly shown in MS patients to be lower than in healthy controls on a population level. The difference, however, is minuscule. Based on current data, it is doubtful that BNDF levels can be of use in any kind of decision-making process regarding either diagnosis or treatment at the patient level. On the other hand, should current results be confirmed by further research, then in the future, the methylation status of the BDNF gene might provide insight into the predicted disease severity of MS patients.

\section{Copeptin}

Copeptin is the more stable C-terminal glycopeptide part of pro-arginine vasopressin (pro-AVP) consisting of 39 amino acids ${ }^{109}$. It is mainly secreted by the paraventricular and supraoptic nucleus of the hypothalamus. Copeptin is found in the plasma in equimolar concentration with AVP of both healthy and critically ill subjects ${ }^{110111}$ and 
can be used as a surrogate biomarker of AVP. The use of copeptin rather than AVP as a biomarker is supported by the fact that it is much more stable and therefore more easily quantifiable than AVP itself ${ }^{112-114}$. Both corticotrophin-releasing hormone (CRH) and AVP are released from the hypothalamus, and they both potently induce the production of adrenocorticotrophic hormone (ACTH) from pituitary corticotrophic cells. ACTH, in turn, stimulates glucocorticoid secretion from the adrenal gland ${ }^{115}$. This set of cascade-like events between the three organs is called the hypothalamopituitary-adrenal (HPA) axis. The HPA axis is initiated and modulated by stressors of different nature ${ }^{116}$. As copeptin is a robust simulator of ACTH secretion, it may be an excellent marker to monitor HPA activity indirectly. Not surprisingly, copeptin has chiefly proven to be a reliable diagnostic marker of cardiovascular diseases (acute myocardial infarction, shock, SIADH, diabetes insipidus) ${ }^{117-119}$ in the pathogenesis of which the dysfunction of the HPA axis and the vasopressinergic system play a pivotal role. In addition to the HPA axis' implication in cardiovascular diseases, various anteand postmortem studies have found a disturbance in the HPA system of MS patients $^{120-123}$. Hyperactivity of the HPA axis has been linked to faster disease progression $^{120}$, and enlarged adrenal glands were found postmortem in MS patients, consistent with increased glucocorticoid production ${ }^{121}$. Furthermore, increased levels of cortisol in the CSF ${ }^{122}$ and a higher amount of CRH and AVP coexpressing neurons in the hypothalamus have been documented. These alterations were linked to a shorter disease length and faster progression ${ }^{123}$. Moreover, increased cortisol awakening response was shown in EDSS progressor RRMS patients, while cortisol levels in patients with a stable EDDS did not differ from healthy controls ${ }^{124}$.

Several studies have demonstrated that copeptin is a viable marker of inflammation and can be used as a prognostic factor of the outcome in different diseases, including those affecting the CNS $116,125,126$.

In light of these results, copeptin's potential value in multiple sclerosis as a biomarker for progression has been raised. To date, only a few papers have explored this possibility ${ }^{127-129}$. The results of these studies, however, are not compelling. Unfortunately, every study examined only a very small population of patients. All of them had a different study setup and employed different measurement protocols. Furthermore, there was significant heterogeneity amongst the enrolled patients across the studies; patients with different disease subtypes were recruited, some studies measured copeptin levels during a relapse. Meanwhile, others examined samples from patients in remission; furthermore, the disease activity, physical disability, comorbidities and other biometric parameters also varied considerably between cohorts. These attributes might have significantly influenced the measured copeptin levels and thus may be responsible for the inconsistent and uncompelling results. Prokopova et al. ${ }^{129}$ have evaluated 19 recently diagnosed MS patients in remission 
before starting their immunomodulatory therapy but after the initial steroid boost for their first relapse. The authors have found mild but prevalent cognitive impairment in the subjects already at the beginning of their disease. Cognitive dysfunction was linked to MS patients (especially in males) having lower BDNF levels than healthy controls. On the other hand, plasma levels of copeptin, cortisol, and aldosterone did not differ between healthy controls and MS patients this early in their disease. This might suggest that the HPA axis hyperactivity - confirmed by other studies - develops later on during the disease. These results have to be taken into account, knowing that patients with obesity or any kind of endocrine disturbance that might influence the HPA axis were excluded from the study ${ }^{129}$. Another study ${ }^{128}$ examined 40 newly diagnosed RRMS patients. All participants were in relapse, therapy-naive, patients with comorbidities that might have influenced cortisol and/or copeptin levels other than obesity were excluded. The MS cohort as a whole was found to have higher copeptin levels compared to HCs. However, this difference had vanished when only lean patients and control subjects were compared and became more prominent when obese patients and controls were evaluated. Furthermore, this comparison observed significantly higher cortisol levels in obese MS patients. When lean and obese MS patients were assessed, no difference was seen in the copeptin levels; however, obese patients were found to have higher cortisol levels than lean patients.

In conclusion, positive correlations were observed between cortisol and copeptin levels in obese MS patients. The results point towards adiposity, not MS itself being the culprit behind the observed alterations of copeptin and cortisol levels in MS patients. The last study addressing the matter ${ }^{127}$ enrolled 30 RRMS patients who were in remission for at least a year (MS controls), 19 RRMS patients who have suffered a relapse within one week in whom the copeptin levels were measured during relapse (MS relapse) and reassessed in a month's time afterwards (MS remission) and 30 healthy controls. Copeptin levels were highest in the MS control group, followed by the MS remission and MS relapse groups. The lowest levels were observed in HCs. No significant correlation was found between the plasma copeptin levels and patients' age, disease duration, or EDSS scores. The results of this study suggest the hyperactivation of the HPA axis in MS patients, which is in line with the findings of several previous papers. Several underlying mechanisms and confounding factors iNfLuence the HPA axis and influence plasma copeptin levels. Based on current results, copeptin does not seem to be a viable and reliable marker of progression or disease course in MS, especially not on a patient level. Further studies with much larger groups of patients and more uniform inclusion-exclusion criteria and analytical methods are necessary to justify and verify copeptin's potential role as a biomarker for MS. 


\section{Chitinase 3-like 1 protein (CHI3L1)}

Chitinase 3-like 1 protein (CHI3L1, also known as YKL-40) encoded by the CHI3LI gene on the long arm of chromosome 1 is a secreted glycoprotein of the glycoside hydrolase family 18 with a molecular weight of $40 \mathrm{kDa}$. CH3L1 is secreted by various cell types including, but not limited to, chondrocytes, macrophages, astrocytes, smooth muscle cells, fibroblast-like cells, synovial cells, activated microglia, to name a few $^{130-134}$. Despite the unknown exact biological function of CHI3L1, it was found to be a key player in inflammation, tissue injury, extracellular tissue remodelling and repair, and fibrosis. This is no surprise, as CHI3L1 was shown to bind to different extracellular matrix constituents and several other molecules regulating the processes above (such as heparin, hyaluronic acid, chitin, CD44, IL-13R subunit alpha-2, TMP219, galectin-3, chemoattractant receptor-homologous 2) ${ }^{130,135,136}$. Altered levels of CHI3L1 are strongly associated with many malignant and non-malignant diseases such as various forms of cancer, arthritis, sepsis, diabetes, liver fibrosis, asthma, dementias, and various neurodegenerative disorders, including MS ${ }^{130,137-143}$. Multiple factors (age, changes in the extracellular matrix, various cytokines and growth factors, stress, inflammation) have been identified to influence the production of CH3L1, many of which are also prominent factors in the pathogenesis of MS ${ }^{130}$. In the CNS, CHI3L1 is most abundantly associated with astrocytes, activated microglia and macrophages, especially in the regions of inflammation and at sites of reactive gliosis ${ }^{131}$.

The first studies exploring CHI3L1 in MS have relied on CSF sampling. However, more recently conducted studies have used blood, making repeat sampling much more accessible and tolerable for the patients. A most recent meta-analysis of the literature $^{144}$ has confirmed that CHI3L1's CSF levels are significantly higher in definitive MS patients than healthy controls ( $n=486$ for MS patients vs 228 for HCs, heterogeneity among the studies was insignificant). Several studies also showed CIS patients to have higher CHI3L1 levels compared to HCs, suggesting its overexpression already from the beginning of the disease, highlighting its potential as a prognostic biomarker. Accordingly, the elevation of CHI3L1 in both the CSF and sera of CIS patients was an independent predictor of both disease conversion and more rapid development of disability ${ }^{145-148}$. On the other hand, there is no significant difference in CHI3L1 levels between RR and progressive subtypes of MS ${ }^{144}$. Similarly, being in remission or in a relapse does not influence the CSF levels of RRMS patients ${ }^{144}$. In contrast, higher levels were associated with increased numbers of T2 and Gd+ enhancing lesions on MRI scans and faster disability progression, fulminant disease course $^{149}$ and hastened spinal cord atrophy ${ }^{150-156}$. Furthermore, CHI3L1 proved to be a reliable marker for distinguishing between $R R$ and progressive phenotype and forecasting disability progression when measured together with neurofilament light chain (NFL) ${ }^{157}$. Natalizumab, fingolimod and mitoxantrone were found to reduce CSF 
levels of CHI3LI1 in RRMS patients, while for interferon-beta, the same was true only for treatment responders ${ }^{153,158,159}$. On the other hand, glatiramer acetateField 156 and dimethyl fumarate (at least in patients with progressive disease)Field 158 did not influence CHI3L1 levels.

Another chitinase family member, $\mathrm{CHI} 3 \mathrm{~L} 2$, has also sparked some interest as a potential biomarker in MS. A pilot study ${ }^{160}$ has found the predictive capacity of CHI3L2 to be similar to that of CHI3L1. Compared to HCs, elevated levels of CHI3L2 were found in the CSF of patients with optic neuritis; also, patients with higher CHI3L2 levels were more likely to develop MS in the future. Furthermore, CHI3L2 was shown to correlate well with the presence of cognitive impairment ${ }^{160}$ and to predict long-term disability progression ${ }^{161}$. Contrasting to CHI3L1, higher CHI3L2 levels at diagnosis were associated with lower baseline EDSS scores in PPMS patients. Furthermore, converse to CH3L1, lower levels of CHI3L2 were measured in patients with a progressive disease than in RRMS patients ${ }^{155}$.

Based on the current data available, both CHI3L1 and CHI3L2 are promising biomarkers in the diagnosis of MS. Furthermore, CHI3L1 seems to correlate well with disease activity and progression in a fashion that DMTs may modify. Strictly speaking, neither CHI3L1 nor CHI3L2 is confined to a specific phenotype of MS. However, differences in their combined levels might be suggestive of a particular disease subtype. Nonetheless, further confirmatory assessment in larger and more homogenous samples of patients with MS are needed to validate CHI3L1's and CHI3L2's status as a biomarker.

\section{C-X-C motif chemokine 13 (CXCL13)}

CXCL13 is a chemokine protein-ligand of the B-cell receptor CXCR5 $5^{162}$. It is one of the most potent B-cell chemoattractants. It is also responsible for the organisation of Bcells within lymphoid follicles ${ }^{163}$ and forming ectopic meningeal B-cell follicles and the meningeal tertiary lymphoid organ, which is crucial in intrathecal autoimmunity and the development of MS ${ }^{164,165}$. CXCL13 is expressed in several tissues, not unexpectedly, the highest concentration is seen in organs with lymphoid tissue, such as the spleen, lymph nodes and the gut ${ }^{166}$. As B-cells are known to contribute significantly to the pathogenesis and progression of MS 167,168, it is not surprising that CXCL13 has gained considerable interest as an auspicious biomarker of the humoral immune response in the CNS. Various studies have documented elevated levels of CXCL13 in the CSF of

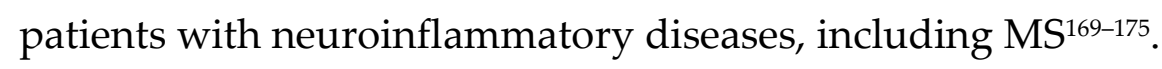

In the past decade, intrathecal CXCL13 has been established as a valuable prognostic biomarker in CIS patients. Elevated CSF CXCL13 levels are well documented to correlate well with CSF cell count, the presence of oligoclonal bands, and IgG index ${ }^{176}$. Furthermore, high CSF CXCL13 concentration was confirmed by several studies to be 
associated with an increased risk of conversion to clinically definite MS (CDMS), a higher relapse rate, also accurately predicted future disease activity ${ }^{171,176-178}$. However, the use of CSF CXCL13 as a biomarker is not restricted to only CIS patients. In RRMS patients, it was shown to correlate with disease activity and indicators of a more severe disease course, such as the relapse rate, IgG index, intrathecal leukocyte count, cortical atrophy and HLA genotype ${ }^{169,171,174,179-181}$. Not surprisingly, CSF CXCL13 levels seem to be a robust and sensitive indicator of intrathecal B-cell response, even under the conditions of an intact blood-brain barrier ${ }^{167,182}$. Accordingly, similarly to RRMS, elevated CSF CXCL13 levels have been associated with disease activity, increased CSF cell counts, IgG-index and MBP, NFL and CHI3L1 concentrations in progressive MS (both PPMS and SPMS) as well ${ }^{183,184}$. Furthermore, as a single marker CXCL13 index

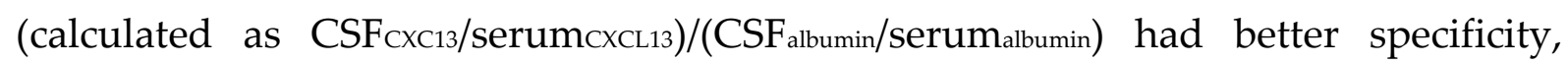
sensitivity, positive and negative predictive value to forecast future disease activity than OCBs and CSF NFL did. Even higher sensitivity and predictive values were achieved when the CXCL13 index and CSF NFL levels were combined ${ }^{181}$. In addition, elevated levels of intrathecal CXCL13 were detected in 50\% of patients treated with highly active DMTs who seemingly had stable disease (no signs of clinical or ongoing radiological activity on MRI), indicating residual, subclinical disease activity ${ }^{185}$. These results further support CXCL13's greater sensitivity to disease activity than clinical and MRI measures.

The tight correlation between CXCL13 and B-cells and other CSF markers of disease severity makes it a perfect candidate to measure the therapeutic efficacy of the B-cell depleting therapies in MS (ofatumumab, rituximab and ocrelizumab) ${ }^{186-188}$. Unfortunately, due to the only recent approval of ofatumumab and ocrelizumab for MS, there are no specific data available yet in the literature regarding these DMTs' effects on CXCL13 levels, except for rituximab, which is currently used off label for MS 189-192. After treatment with rituximab, two chemokines, CXCL13 and CCL19, were shown to significantly decrease in the CSF of patients in correlation with reduced Bcell and T-cell numbers ${ }^{193,194}$. Regarding other MS therapies, CXCL13 has been reported to decrease in patients after treatment with natalizumab or methylprednisolone ${ }^{152,169,195,196}$, mitoxantrone ${ }^{197}$, but not after interferon-beta treatment ${ }^{152}$. Furthermore, baseline CXCL13 levels were also shown to predict success with fingolimod; nonresponder patients had elevated pretreatment serum levels of CXCL13 compared to patients responsive to fingolimod ${ }^{198}$.

Compelling amount and quality of evidence suggest that CXCL13, especially in CIS patients, is a valuable and reliable prognostic marker of conversion probability and future disease activity. CXCL13 and CXCL13 index seem to be excellent markers for disease activity and severity in other disease subtypes. Furthermore, if confirmed by 
studies employing larger cohorts CXCL13 may have broader utility as a biomarker of therapeutic response, especially in patients on B-cell therapies.

\section{Osteopontin (OPN)}

Osteopontin (OPN, also known as early T-lymphocyte activation [ETA-1]) is a secreted, negatively charged glycoprotein rich in attached carbohydrate and sialic acid residues, composed of $\sim 300$ amino acids encoded by the SPP-1 (secreted phosphoprotein 1) gene on the long arm of chromosome $4^{199200201}$. OPN is an extracellular matrix protein secreted by a variety of cell and tissue types (such as osseal cells, fibroblasts, myoblasts, the placenta, smooth muscle and endothelial cells and several cells of the immune system including dendritic cells, natural killer cells, T-cells and macrophages), that is associated with various physiologic processes (bone mineralisation and remodelling, wound healing, chemotaxis, immune cell activation, apoptosis regulation) and pathologic states (cancer biology, vessel wall stiffness and atherosclerosis, cardiovascular, pulmonary, neurodegenerative and inflammatory diseases) ${ }^{201-203204}$. On the one hand, OPN looks to be a principal contributor to inflammation resulting in tissue damage. On the other hand, it seems to be a key player in the subsequent reparative mechanisms triggered by the inflammation itself ${ }^{204,205}$. Via several mechanisms and pathways ${ }^{206-208}$ OPN shifts the cytokine balance towards the proinflammatory side. It increases the production of IL- $1 \beta$, IL- 12, IL-17, IFN- $\gamma$ and inhibits the expression of IL-10, resulting in detrimental neuroinflammation and the inhibition of lymphocyte death ${ }^{209-211}$. In light of this, and as OPN is widely expressed by cells resident in the CNS (virtually on all neurons and glia), and by various immune cells that are either already present in or are capable of migrating into the $\mathrm{CNS}^{208,212}$, it is not surprising for it to be involved in MS pathology. This theory is supported by the confirmed overexpression of OPN in MS lesions ${ }^{213}$, the association of its gene variations with MS susceptibility and progression 214,215 , and the plethora of studies reporting elevated levels of OPN in the blood and CSF of MS patients ${ }^{204,205}$.

A most recent meta-analysis ${ }^{204}$, based on 27 studies, concluded that MS patients irrespective of disease subtype have higher CSF and blood OPN levels than HCs or other patients with non-inflammatory neurological disorders (NIND). When stratified by disease subtype, RRMS patients were found to have the highest CSF OPN levels, followed by CIS and SPMS patients. PPMS patients were shown to have higher CSF and blood OPN levels than CIS patients ${ }^{204}$. Furthermore, higher CSF OPN levels were linked to a higher degree of disability in PPMS ${ }^{216}$. Additionally, concomitantly increased CSF OPN and CXCL12, but not IL-10 levels were measured in PPMS patients compared to RRMS patients ${ }^{217}$. CSF OPN levels were demonstrated to correlate with disease activity; CSF concentration of OPN was shown to elevate in exacerbation of the disease irrespective of disease subtype and subside after the resolution of the attack regardless of whether steroid therapy was administered or not ${ }^{216}$. Accordingly, 
patients with stable disease had lower CSF OPN levels than patients who showed activity $^{204}$. Despite these alterations in the OPN levels between different MS subtypes, the meta-analysis did not find any difference in the CSF OPN levels of MS patients and patients with other inflammatory neurological diseases ${ }^{204}$ No other comparison between MS subtypes has shown a significant difference in blood and/or CSF OPN levels between the examined groups. Another study has found that higher CSF (but not serum) OPN levels at baseline predicted higher white matter lesion volume and white matter loss, increased cortical/subcortical grey matter atrophy, ventricle enlargement and various microstructural alterations in the NAWM of MS patients seen on MRI scans performed more than a decade later 218,219 .

Studies in the literature have demonstrated that circulating or CSF osteopontin levels are neither specific enough to differentiate MS from other inflammatory diseases affecting the CNS. Moreover, in contrast to the conformity of results on the higher CSF OPN found in MS patients vs HC results regarding serum OPN levels in MS patients are somewhat conflicting ${ }^{148,220}$. Overall neither CSF nor serum osteopontin is likely to be useful in the everyday clinical setting as a diagnostic biomarker ${ }^{221,222}$. On the other hand, despite a lack of specificity for MS ${ }^{223}$, both CSF and serum osteopontin levels correlate significantly with inflammation, disease activity and clinical severity ${ }^{216,224-226}$. Additionally, CSF osteopontin levels might be valuable biomarkers for therapeutic efficacy as they were shown to respond to treatment with natalizumab and interferon$\beta^{195,227-229}$. The data from studies suggests that CSF osteopontin, together with other CSF biomarkers of inflammation, may be used to monitor therapeutic effects on intrathecal inflammation. However, additional studies are required to confirm these results with other currently used DMTs and validate OPN as a disease activity biomarker in the blood.

\section{Neurofilament proteins - background}

Neurofilaments (Nfs) are presumably the most studied potential biomarker of diseases affecting the nervous system. Nfs are considered tissue-specific type IV class intermediate filaments since their diameter $(10 \mathrm{~nm})$ falls between actin $(6 \mathrm{~nm})$ and myosin $(15 \mathrm{~nm})$ filaments and are responsible for the cytoskeletal integrity and structure of neurons of the central nervous system (CNS) as well as the peripheral nervous system $(\mathrm{PNF})^{230}$. Nfs are predominantly located in large, myelinated axons of the white matter (WM), yet a less intense expression of these proteins also appears in the grey matter $(\mathrm{GM})^{231}$. In mature neurons, five different neurofilament proteins are responsible for cytoskeletal stability: peripherin, alpha-internexin, neurofilament heavy chain $(\mathrm{NfH})$, neurofilament medium chain (NfM), and neurofilament light chain (NfL). Nfs in PNF neurons constitute NfL, NfM, NfH and peripherinField 231, while in CNS neurons, Nfs compose NfL, NfM, NfH, and alpha-internexin ${ }^{232}$. The initial step 
of synthesising these neurofilament proteins is dimer and tetramer formation, which generally consists of NfLs and another Nf protein ${ }^{233}$. Synthesis usually takes place in the cell body, and transportation to axons and dendrites happens as hetero-oligomers and shorts filaments ${ }^{234}$. After reaching their final destination, these smaller compounds form stable neurofilament networks specific to the region ${ }^{235}$. Even though the exact function of Nfs continues to be concealed, they are assumed to play a predominant role in axon stability ${ }^{236}$ contributing to adequate nerve conduction velocity ${ }^{237}$, and in recent years it has been proven that Nfs also engage in appropriate synapse functioning as well ${ }^{238}$. Mutations in genes encoding neurofilaments or causing neurofilament accumulation and aggregation have been linked to several diseases of the CNS and PNS characterised by axonal dysfunction and neurodegeneration, such as Parkinson disease (PD), amyotrophic lateral sclerosis (ALS) ${ }^{239}$ and Charcot-MarieTooth disease ${ }^{240}$. Fundamentally, any pathophysiological process or disease resulting in axonal damage, whether it is acute or chronic in character, can result in increased levels of Nfs. Thus, monitoring changes in Nf levels has a potential relevance as a biomarker in neurological disorders, such as traumatic brain injury, stroke, ALS, PD, Alzheimer's disease, Huntington's disease, and multiple sclerosis (MS) dissected by numerous studies ${ }^{241}$. Because of the tissue-specific nature of Nfs, it is comprehensible that these molecules have been the centre of attention in the past three decades ${ }^{230}$, and especially studies investigating NfL have demonstrated promising results ${ }^{242}$. NfLs are assumed to enter the CSF by direct consequence of neuronal cell membrane disruption, yet other mechanisms, for example, active secretion or multivesicular bodies, might also play a role in NfL release ${ }^{243}$. NfL peptides are thought to enter the bloodstream primarily through intramural periarterial drainage and the glymphatic system $^{244}$. According to various studies, CSF and blood NfL levels show a significant correlation ${ }^{245,246}$. Nonetheless, while CSF NfL levels are principally influenced by the location and the extent of the injury ${ }^{247}$, blood NfL levels may also be affected by the integrity of the blood-brain barrier $(\mathrm{BBB})^{248}$, PNS damage ${ }^{249,250}$, cardiovascular risk factors $^{251}$ and increased blood volume due to high body mass index ${ }^{252,253}$. Additionally, low concentrations of NfL can be constantly detected in different body fluids, suggesting that NfL is released into CSF and blood as part of physiological processes, such as ageing-related neurodegeneration. Research indicates that BBB integrity might decrease with ageing ${ }^{254}$ further supports this hypothesis. This age-specific increase was recently determined in healthy individuals as a $2.2 \%$ rise per year in blood NfL levels ${ }^{255}$. Furthermore, decreasing renal function associated with the elderly might also influence blood NfL concentrations ${ }^{256-258}$. Thus, this factor should also be considered when interpreting NfL levels. 
Ultimately, NfL might be a valuable diagnostic ${ }^{259}$, differential diagnostic ${ }^{260}$, prognostic biomarker ${ }^{261,262}$, and could also help predict the outcome 263,264 , monitoring disease activity and therapeutic response ${ }^{263}$ in a variety of neurological disorders. Of the aforementioned neurological disorders, NfL might have been the most thoroughly investigated as a potential biomarker concerning multiple sclerosis.

\section{Neurofilament light chain in MS}

The first assumption that NfL might be a potential body fluid biomarker in MS dates back to 1998. Lycke and colleagues examined CSF NfL levels of 60 persons with relapsing-remitting MS (pwRRMS) compared to healthy controls in the course of 2 years. They discovered that individuals with RRMS had significantly elevated NfL levels that correlated with disability and future relapses ${ }^{265}$. And even though NfL is not specific to MS, it has been extensively studied in the past two decades in this field. Several studies dissected the potential role of NfL use in MS regarding diagnosis, prognosis, disease activity and therapeutic response monitoring.

Initial studies reported elevated levels in MS compared to healthy controls (HCs). Studies reported equal levels of NfL among MS subtypes ${ }^{266,267}$, others found elevated NfL levels in RRMS compared to progressive MS 268,269 , suggesting that NfLs get released into the CSF during acute inflammatory activity. In contrast, a few surveys reported that NFL levels were more elevated in PMS compared to RRMS 270,271 , indicating that NfL release is also involved in neurodegenerative processes. Elevated NfL levels have been reported with the first demyelinating event in the paediatric population. Furthermore, higher NfL levels were associated with future conversion to RRMS $^{272}$ and the early postpartum period ${ }^{273}$. Subsequently, due to the more widespread availability and use of DMTs, sporadical surveys could not identify any difference between NfL levels in MS and $\mathrm{HCs}^{29}$. Increased NfL at baseline or during follow-up might represent inflammatory activity, as several investigations declared a correlation with relapses, the occurrence of new T2 hyperintense and Gadolinium enhancing lesion ${ }^{274,275}$. High NfL levels may also reflect progression since several studies reported an association between high NfL concentration and T1 hypointense lesions and brain atrophy ${ }^{271,276}$. The associations above also raised the possibility that NfL may be suitable to complement MRI examinations, which are nowadays are the most sensitive in monitoring disease activity and therapeutic response. Implementing NfL measurement into everyday clinical practice as disease activity and therapy response monitoring tool would involve regular sampling. Even though CSF contains the most considerable amount of NfL and CSF NfL concentrations are the most sensitive to insults occurring in the $\mathrm{CNS}^{277}$ because of the invasive nature of lumbar puncture and the fact that NfL eventually enters the circulation via lymphatic drainage or directly in consequence of BBB disruption associated with a demyelinating event ${ }^{248}$, there has been an increasing emphasis to detect NfL from blood reliably. The first 
attempts facilitated ELISA ${ }^{278}$, later endeavours exploited electrochemiluminescence $(\mathrm{ECL})^{275}$, and in recent years the ultrasensitive single-molecule array (SiMoA) 279,280, which became the golden standard technique. The majority of these assessments focused on serum samples ${ }^{178,275,281,282}$; however, a few utilised plasma ${ }^{283-285}$, or both ${ }^{277}$ to examine correlation with CSF samples. Despite the different procedures, a significant correlation was ascertained about CSF and serum ${ }^{286266,282}$, as well as CSF and plasma samples $^{285}$, and CSF, serum and plasma samples ${ }^{277}$. Other investigations, however, documented a weak correlation between these sample types ${ }^{284}$. A study compared the three techniques regarding the correlation between CSF and serum samples. SiMoA was revealed to have the highest analytical sensitivity, and CSF and serum samples measured by SiMoA showed the strongest correlation ${ }^{245}$. Another problem with NfL measurements was the low comparability of results due to great inter-facility discrepancies ${ }^{287}$. Sejbeak et al. ${ }^{287}$ addressed this topic and have found that NfL levels measured by SiMoA in CSF and plasma in different institutions were comparable since variability was minor and mainly affected values close to the cut-off. However, they also concluded that variability in the laboratory methods might result in more significant discrepancies, making interpretation of values between facilities impractical, thus suggested the standardisation of measurement methodology.

As previously mentioned, NfL levels correlate with age in HCs: in a cohort inspecting 335 healthy individuals, median serum NfL levels were quite similar in the 4th (18.90 $\mathrm{pg} / \mathrm{ml})$ and 5 th $(22.10 \mathrm{pg} / \mathrm{ml})$ decades but then increased nonlinearly in the 6th (32.4 $\mathrm{pg} / \mathrm{ml}$ ) and 7 th decade $(43.3 \mathrm{pg} / \mathrm{ml})$, which correlated with mean changes in brain atrophy with a $0.9 \%, 2.7 \%, 4.3 \%$ and $4.3 \%$ change between the ages of $40-50,50-60,60-$ $70,>70$ respectively, showing stabilisation above a certain age, however, NfL concentration did not correlate with gender ${ }^{288}$. In MS, multiple studies investigated the association between NfL levels and age and gender with miscellaneous results. Most surveys failed to identify any correlation between CSF and serum NfL and age in MS'266,269,271,277,289. Some assessments examining NfL levels in MS compared to HCs found no correlation between age and CSF NfL in MS but strengthened the association mentioned above with age in $\mathrm{HCs}^{279,290,291}$. On the other hand, different studies documented a significant relationship between age and CSF, serum, and plasma NfL in $\mathrm{MS}^{40,15,283,292}$. The association between NfL and gender were also examined several times. Most studies found no relationship between CSF and serum NfL and $\operatorname{sex}^{40,276,292,293}$. On the other hand, a cohort reported higher serum NfL levels in females ${ }^{294}$, while according to other studies, CSF NfL concentrations were more prominent in male patients ${ }^{295,296}$.

\section{Diagnosis}

Numerous surveys assessed the utility of NfL in the diagnosis of MS. Since NfL is not specific to MS, its concentration can rise above normal in other inflammatory 
neurological diseases and other noninflammatory neurological disorders. Thus, NfL per se is not feasible to diagnose $\mathrm{MS}^{297}$. According to the latest diagnostic guidelines, diagnosis of MS can be established if clinical symptoms and/or paraclinical findings support dissemination in time and space ${ }^{8}$, and even though the currently used criteria significantly reduced the time previously needed to reach a diagnosis, in individuals with radiologically (RIS) and clinically isolated syndrome (CIS) clinicians still can encounter diagnostic difficulties. However, NfL could be a valuable tool to supplement and fasten existing diagnostic processes to reduce the time from first clinical event to diagnosis and predict future conversion from RIS and CIS to clinically definitive MS (CDMS) discussed hereunder.

A recent study examining 75 RIS patients found elevated CSF NfL levels in RIS converters compared to nonconverters ${ }^{298}$, suggesting higher NfL levels might be a good predictor of future conversion. Another survey investigated CSF NfL and progranulin concentration in an MS cohort comprising RIS, CIS, RRMS, PPMS patients and HCs. It identified elevated NfL levels in CIS, RRMS and PPMS patients. However, it could only reveal significantly elevated progranulin but not NfL levels in RIS patients compared to $\mathrm{HCs}^{299}$.

Various studies described elevated CSF and serum NfL levels in CIS compared to $\mathrm{HCs}^{279,299-302}$ and other non-inflammatory neurological disorders ${ }^{290}$. A recent survey conducted on 177 newly diagnosed CIS and RRMS patients showed increased CSF NfL levels in both groups. There was no statistically significant difference between group NfL levels ${ }^{303}$, further supported by others ${ }^{290}$. In contrast, another study described higher CSF NfL levels among RRMS patients compared to patients with CIS ${ }^{279}$. Multiple investigations focused on the predictive value of NfL concerning CIS conversion to CMDS. A prospective study from Netherland inspecting paediatric and adult CIS patients reported elevated CSF NfL levels, with higher NfL levels indicative of future conversion to RRMS in both populations ${ }^{301}$. Similarly, a retrospective survey including CIS patients described higher NfL levels among CIS converters compared to nonconverters ${ }^{275}$. In addition, according to a study examining 32 patients with a first demyelinating event, higher baseline CSF NfL levels predicted future relapse and diagnosis of $\mathrm{CDMS}^{304}$. CSF NfL levels in combination with other cytokines were also shown to differentiate between isolated optic neuritis and patients later converting to $\mathrm{CDMS}^{178}$. These convincing results were refuted by other studies, however. Avsar et al. revealed higher GFAP and Tau, but not NfL levels among CIS converters ${ }^{300}$. Distano and colleagues reached a similar verdict. Their results show that more elevated serum NfL levels did not correlate with faster conversion to $\mathrm{CDMS}^{302}$. To add to the controversy, a retrospective survey from the US conducted on 120 military persons diagnosed with MS found that serum NfL levels of these individuals were elevated years before diagnosis, and a within-person increase was associated with shorter time 
to clinical onset ${ }^{305}$. Ultimately, in case of uncertainty, measurement of NfL might be utilised as a complementary examination to distinguish patients at risk of developing CDMS from non-converters. In addition to identifying CIS patients with risk of future conversion higher baseline CSF NfL is associated with disease severity ${ }^{268}$, future EDSS progression and conversion to SPMS ${ }^{293,295}$. Data in the literature suggests that not baseline ${ }^{268}$ serum NfL but the change in its level indicates future conversion to SPMS ${ }^{306}$. All in all, evidence suggests that baseline and repeat NfL measurement might complement current clinical and paraclinical evaluations to identify patients at risk of conversion. According to a 15-year longitudinal follow-up study, baseline serum NfL levels above $7.62 \mathrm{pg} / \mathrm{ml}$ can predict future conversion to SPMS with a 93.3\% sensitivity and $46.1 \%$ specificity ${ }^{307}$.

Another diagnostic dilemma not entirely resolved by routine clinical and MRI examinations is accurately establishing PML risk in JC positive patients receiving natalizumab (NAT). A study investigating 96 patients who received NAT showed that even though serum NfL levels at therapy initiation were similar in patients who later developed PML and patients who did not, NfL levels were reduced in both groups during treatment. However, when measured later in the second year, patients who later developed PML had significantly higher NfL levels than the rest of the cohort ${ }^{308}$. This is further supported by a case report of two patients with PML who showed continuous elevation of serum NfL levels at PML onset, which increased further in a patient having IRIS; however, after treatment, parallel to subsiding PML, serum NfL levels decreased as well ${ }^{309}$. Thus, NfL might be a helpful candidate in monitoring patients at risk of PML and diagnosing PML.

\section{Monitoring disease activity}

As mentioned above, according to numerous studies, elevated NfL levels are associated with clinical and radiological disease activity. At the same time, low concentrations comparable to HCs indicate a stable disease. Moreover, longitudinal studies suggest that NfL levels measured at baseline and throughout follow-up predict future changes. These attributes make NfL a good candidate for monitoring disease activity.

\section{Relapse activity}

Countless studies revealed that CSF NfL levels were associated with relapses implying that axonal damage was most pronounced in the presence of disease activity ${ }^{152,185,268,310}$. High baseline serum NfL levels were shown to correlate with relapse activity of the previous year ${ }^{271,303}$ and forecast a relapse in the near future (up to 90 days), but not in the subsequent years after sampling ${ }^{311}$. High baseline levels are also associated with the number of future relapses as well ${ }^{304,312}$. Not just flat levels, but elevating kinetic of NfL can also indicate future clinical and radiological disease activity ${ }^{313,314}$. CSF NfL levels remaining high after one year of DMT treatment were shown to convey relapse 
risk and predict therapeutic ineffectivity ${ }^{157}$. In contrast to these results, others examining CSF NfL ${ }^{289}$ and serum NfL concentration ${ }^{315}$ failed to establish any correlation between baseline NfL levels and ongoing ${ }^{316}$ or future relapse activity ${ }^{317}$.

Disability: EDSS, Timed 25 Feet Walk Test (T25FWT) and 9-Hole Peg Test (9HPT)

According to cross-sectional studies, high baseline CSF NfL levels correlate with high baseline EDSS 156,290 and future EDSS progression $281,293,295$. Somewhat contradictory to this data are the results of other studies that confirmed the correlation between baseline $\mathrm{CSF}^{303}$, serum ${ }^{294}$ and plasma ${ }^{318}$ NfL levels and baseline EDSS but failed to link them to future EDSS and disability progression. Some studies documented a link between baseline serum NfL and baseline EDSS248,274,294,302,316, while others did not find such correlation ${ }^{319-321}$. In contrast, a few investigations could not reveal any association at all between $\mathrm{CSF}^{40,157,271,322}$ or blood ${ }^{277} \mathrm{NfL}$ levels and EDSS. Interestingly another study demonstrated no correlation between baseline serum NfL levels and baseline EDSS; however, high baseline serum NfL concentrations predicted high EDSS levels at follow-up and at study end ${ }^{323}$. Not just flat concentrations, but the change in serum NfL levels has also been associated with a change in EDSS ${ }^{311,315}$ scores. Furthermore, falling CSF NfL levels after DMT initiation are linked to stabilising EDSS scores ${ }^{292}$.

In SPMS, higher serum NfL was linked to hastened whole-brain atrophy, more new/enlarging T2 lesions and higher T2 lesion volume. In contrast, NfH levels showed no correlation with any clinical or MRI measures ${ }^{324}$. A retrospective study evaluated the serum and CSF NfL concentrations of a population participating in an interferonbeta treatment study, has found that both CSF and serum NfL levels taken at years 2, 3 , and 4 were predictive of patients reaching EDSS 6 at 8 and 15 years of follow-up ${ }^{281}$.

Data are limited regarding the association between NfL concentration and disability measured by the Timed 25 Feet Walk Test (T25FWT) and 9-Hole Peg Test (9HPT). Some found no correlation between serum baseline NfL levels and baseline $9 \mathrm{HPT}^{315}$ or T25FWT at baseline ${ }^{315}$ or after ten years of follow-up ${ }^{325}$. Others, however, have found that higher baseline serum NfL correlated well with baseline physical disability measured by 9HPT and worse performance in the T25FWT at follow-up ${ }^{324}$.

MRI activity

$\mathrm{T} 1, \mathrm{~T} 2$ and Gadolinium enhancing lesions

As mentioned earlier, several studies demonstrated a correlation between NfL levels and evidence of radiological activity. Baseline CSF NfL levels are associated with baseline ${ }^{290}$, and future Gd+ lesions ${ }^{304}$. Similarly, CSF NfL levels were shown to correlate not just with the presence of Gd+ lesions ${ }^{185,296,326}$, but the number of $\mathrm{Gd}+$ lesions ${ }^{293,327}$ as well. In contrast, another study found a correlation only between CSF NfL levels and the presence, but not the number of $\mathrm{Gd}+$ lesions ${ }^{157}$. Studies examining serum NfL levels also described an association between baseline serum NfL levels and 
baseline $^{248,274,275,281}$, and future Gd+ lesions ${ }^{328}$. Moreover, an increase in serum NfL levels was associated with the appearance of new $\mathrm{Gd}+$ lesions $^{314,315,329}$. Interestingly in a longitudinal assessment, baseline NfL levels correlated with the presence and volume of baseline Gd+ lesion; however, serum NfL levels at 52 weeks was not associated with $\mathrm{Gd}+$ lesions at 52 weeks $^{320}$. Similarly, another survey found no correlation between serum NfL levels and the number of Gd+ lesions ${ }^{276,330}$.

Several papers evaluated the association between NfL levels and the presence, number and volume of $\mathrm{T} 2$ lesions with mixed results. CSF NfL levels were shown to correlate with both baseline and future ${ }^{281} \mathrm{~T} 2$ lesion load and predict new T2 lesions as well277,279,281. Similarly to CSF, serum NfL levels also correlated with the presence and number of T2 lesions ${ }^{248,272,311}$, vice versa T2 lesion volume showed correlation with baseline serum NfL concentrations ${ }^{276,324}$. Furthermore, some have showed that the rise of serum NfL levels can predict the progression of T2 lesion load months in advance ${ }^{314}$, meanwhile, others failed to confirm these results ${ }^{315}$. This might be explained by the findings of another study, which similarly found no correlation between serum NfL and T1, T2 lesion volumes, measured by conventional MRI. However, high serum NfL levels correlated with T2 lesion volume and normal-appearing white matter (NAWM) damage measured by DTI ${ }^{316}$. Similar results are available regarding T1 lesions; CSF NfL levels correlate with the presence ${ }^{301}$ and volume ${ }^{271}$ of $\mathrm{T} 1$ hypointense lesions. Similarly, serum NfL levels correlate with T1 lesion count ${ }^{272}$, volume ${ }^{274}$ and future accrual of lesions ${ }^{276}$.

\section{Brain atrophy}

There is rapidly rising amount of data assessing the connection between blood/CSF NfL levels and brain atrophy. Not surprisingly, a strong correlation between high CSF NfL concentrations and brain atrophy has been established by several studies ${ }^{27,281}$. Furthermore, one study demonstrated a correlation between CSF NfL levels and grey matter (GM) atrophy ${ }^{156}$. At the same time, another research found CSF NfL levels to correlate only with thalamus and nucleus accumbens volumes, but not whole brain, white matter (WM), GM or putamen atrophy ${ }^{331}$. Nonetheless, in many cases, serum NfL levels were associated with baseline and future brain atrophy $248,276,311,324$. Accordingly, both baseline serum NfL levels and subsequent changes in NfL levels were shown to predict changes in brain volume ${ }^{332}$. This is somewhat contradicted by the findings of Kuhle et al., who have documented that only baseline serum NfL levels, but not the changes in NfL levels correlate with brain atrophy ${ }^{315}$.

\section{Cognitive impairment}

As the knowledge regarding neuropsychological symptoms in MS expands, the clinicians' objective has shifted from solely diagnosing cognitive impairment, fatigue or depression to monitoring and treating these symptoms. The measurement and evaluation of neuropsychological symptoms are based on neuropsychological 
batteries and patients reported outcomes. However, it has been suggested that a correlation between NfL levels and neuropsychological symptoms might exist. Accordingly, high serum NfL levels and cortical thickness have been reported to correlate with global neuropsychological performance measured by Brief Repeatable Battery of Neuropsychological Tests (BRB-N) and Paced Auditory Serial Addition Test $(\mathrm{PASAT})^{330}$. In line with these results, a study on 39 patients showed an inverse association between CSF NfL levels and Brief International Cognitive Assessment for MS (BICAMS) test results in progressive but not RRMS ${ }^{289}$. Confirming these findings, CSF NfL levels were shown by another study to correlate with not just overall cognitive impairment but impairments in information processing speed and verbal fluency as well in newly diagnosed CIS and RRMS patients ${ }^{333}$. In contrast, others found no correlation between CSF NfL levels and cognitive impairment measured by the BICAMS battery 326,334 (a weak correlation was observed with the California Verbal Learning Test [CVLT-II] part of the BICAMS test, however ${ }^{334}$ ). Interestingly an increase in serum NfL levels was associated with worse performance on parts of the BICAMS test but a better score on the PASAT ${ }^{315}$. This phenomenon, however, might be explained by the generally high PASAT scores (50-60 points) reported at both baseline and throughout follow-up ${ }^{315}$. This is supported by another study demonstrating a weak correlation between baseline serum NfL levels and future PASAT scores ${ }^{321}$.

Data are scarce regarding fatigue, depression, and quality of life, but a study reported an association between serum NfL levels at one year and fatigue score worsening at ten years ${ }^{325}$. Yet, another study assessing 38 newly diagnosed CIS and RRMS patients reported no association between serum NfL levels and fatigue ${ }^{335}$. Similarly, another study found no correlation between CSF NfL levels and anxiety or depression ${ }^{336}$. In contrast, a significant correlation was made between baseline serum NfL levels and baseline quality of life measured by the Multiple Sclerosis Quality of Life-54 (MSQoL54) questionnaire, moreover serum NfL levels at baseline and follow-up correlated with changes in MSQoL-54's physical role limitations and social functioning composite scores ${ }^{294}$.

\section{Monitoring therapeutic response}

Another area where regular NfL measurement could be utilised is therapeutic response monitoring. As of today, the most sensitive method for this purpose is an MRI examination performed annually or, in case of relapse, emergently. However, as mentioned earlier, on the one hand, the MRI device itself is expensive and a scarce resource, so it might not be universally available. On the other hand, the examinations are costly; thus, performing regular annual examinations without the occurrence of new or worsening symptoms might not be financed. In this case, another similarly sensitive method for monitoring therapy response may be helpful. Furthermore, disease activity may not always manifest in clinical relapse or radiological changes. In 
some cases, an increase in NfL levels might be the only sign of disease progression in a patient otherwise showing no disease activity ${ }^{185}$. In such situations, complementing MRI examination with regular NfL measurement may provide additional information to make a therapeutic decision. Thus, the dynamics of NfL concentrations during disease-modifying treatment have been extensively studied to understand better how NfL measurements can be implemented in clinical practice to monitor therapeutic response.

\section{Moderately effective DMTs}

Evaluation of patients originally participating in the phase 3 IFN- $\beta$ clinical trial and its extension studies reported significantly reduced CSF NfL levels in patients treated with IFN- $\beta$ compared to placebo. Furthermore, an increase in NfL levels among patients treated with IFN- $\beta$ was associated with a suboptimal treatment response ${ }^{281}$. Another study examined 32 treatment-naïve RRMS patients initiating either glatiramer acetate (GA) or INF- $\beta$. After treatment started decreasing, and then consistently low NfL levels were documented in therapy-responsive patients. However, NfL levels remained high in nonresponders and correlated with MRI and relapse activity ${ }^{321}$.

The effect of dimethyl-fumarate (DMF) on CSF, serum, and plasma NfL levels was assessed in a cohort of 104 previously treatment-naïve RRMS patients receiving either treatment or placebo. At baseline, RRMS patients had higher NfL levels than HCs. After one year of treatment, CSF, plasma, and serum NfL levels all had been reduced to levels comparable to that measured in $\mathrm{HCs}^{277}$. Even though a tight correlation was observed between sample types, CSF NfL levels proved most sensitive to relapse and MRI activity ${ }^{277}$. Another trial conducted with DMF employed 54 PPMS patients (27 on DMF, 27 on placebo). CSF NfL levels were reported to be elevated in both arms, but no clinically significant difference in mean NfL change was seen at the end of the follow-up ${ }^{337}$. A pilot study investigating delayed-release DMF in SPMS patients found that CSF NfL levels correlated better with clinical improvements experienced by patients than MRI ${ }^{338}$.

\section{Highly effective DMTs}

Several studies evaluated NfL in patients receiving natalizumab (NAT). An already significant decrease in CSF NfL concentrations was demonstrated after just 12 months of treatment with NAT in RRMS patients ${ }^{39,339}$. Compared to interferon- $\beta$, patients receiving NAT experienced a more significant reduction in CSF NfL levels, supporting previous results that NAT is more effective in preventing axonal damage than moderately effective therapies ${ }^{152}$. Another study reported similar results; CSF NfL levels were significantly reduced in the whole study population receiving NAT for 12 months. However, a surge in NfL levels was observed in the event of a relapse. At the 
same time, NfL concentrations remained stable in patients with stable disease, further underlining NfL's potential in monitoring therapeutic response ${ }^{340}$. Moreover, according to the ASCEND phase III study, NAT was able to decrease serum NfL levels in patients with active progressive MS as well ${ }^{341}$. Additionally, according to a small cohort conducted on 11 NAT-treated patients during and after pregnancy. CSF and serum NfL levels during pregnancy remained as low as preconception concentration. However, in the early postpartum period, a transient NfL peak was observed that did not correlate with relapse or MRI or clinical activity, suggesting that NAT was effective in preventing disease activity even in pregnancy ${ }^{273}$. As mentioned previously, serum NfL levels during NAT treatment also correlated with PML risk ${ }^{308}$, furthermore showed a significant increase in case of PML onset ${ }^{342}$. Thus NfL levels might not only aid monitoring therapeutic response but may efficiently augment the decision of NAT cessation in JC positive individuals at risk of developing PML.

Different doses of fingolimod (FG) also proved effective in decreasing CSF NfL levels. The change correlated with MRI parameters and relapse rate as well ${ }^{343}$. A study examined patients who either escalated to FG from first-line treatments or switched laterally from NAT. In the case of escalation, CSF NfL showed a significant reduction; however, in the case of changing from NAT, NfL levels remained persistently low. This suggests that NfL might not only play a role in monitoring treatment response but also inform about treatment efficacy ${ }^{159}$. A similarly significant decrease in NfL levels after escalation from injectable therapies to FG was also demonstrated in another study $^{344}$. The reduction in NfL levels also correlated with patients' multiple sclerosis severity scale (MSSS) score. Fingolimod also successfully decreased plasma NfL levels of PPMS patients compared to placebo ${ }^{345}$.

A most recent study evaluated the effectiveness of subcutaneous cladribine (CLA), reported significantly reduced CSF NfL levels at follow-up in a subpopulation of patients with elevated baseline NfL levels ${ }^{346}$. A case report examining patients with progressive MS receiving cladribine reinforced these findings; CSF NfL levels were reduced at follow-up ${ }^{347}$, suggesting that cladribine might be a potential candidate in the treatment of PMS showing disease activity and that NfL levels might reflect therapeutic response in PMS as well.

According to a study examining serum NfL levels of RRMS population participating in the CARE-MS and extension studies, compared to baseline measures, alemtuzumab significantly reduced NfL levels at two years which was sustained at seven years. Moreover, NfL levels at the end of the observation period were significantly lower in alemtuzumab treated patients compared to patients receiving IFN- $\beta^{274}$. Furthermore, in a small cohort of 15 highly active MS patients, elevated baseline serum NfL levels 
continuously decreased until reaching a stable state after treatment with alemtuzumab. The low NfL levels were coupled with a drop in disease activity measured by the annualised relapse rate and MRI parameters. Accordingly, low NfL levels correlated with no evidence of disease activity, whereas an increase in NfL levels was associated with progression of T2 lesion load and appearance of new Gd enhancing lesions ${ }^{314}$. In a large cohort, compared to DMF, FG, NAT, teriflunomide and rituximab, alemtuzumab was associated with the lowest on-treatment plasma NfL levels and highest reduction in NfL levels compared to baseline ${ }^{348}$.

The ORATORIO phase 3 randomised clinical trial compared ocrelizumab effectively decreased the rate of disability progression and serum NfL levels compared to placebo $^{349}$; however, real-world clinical data on ocrelizumab are scarce, future observational studies are needed.

The EXPAND Phase 3 study examined the efficacy of siponimod in SPMS patients. It revealed that after 21 months of follow-up, serum NfL levels in patients receiving treatment were notably decreased, while serum NfL levels in patients in the placebo arm were even increased compared to baseline ${ }^{350}$.

In the ASCLEPIOS clinical trial, ofatumumab significantly reduced serum NfL levels at follow-up compared to baseline; however, the comparator, teriflunomide, only showed a modest decrease in serum NfL levels. Moreover, ofatumumab appeared to be superior to teriflunomide in disability and MRI related endpoints as well ${ }^{351}$.

\section{Other therapies}

Elevated baseline CSF NfL levels were reported in a cohort of 46 patients receiving autologous haematopoetic stem-cell transplantation (aHSCT). CSF NfL levels decreased significantly after aHSCT ${ }^{312}$. Another study that included 23 patients with aggressive MS supported this finding. After HSCT, CSF and serum NfL levels significantly decreased and remained low in patients who responded to therapy but have remained high in nonreponders ${ }^{276}$.

Similarly, a significant decrease in CSF NfL levels was observed in patients who switched to rituximab from either IFN- $\beta$ or glatiramer acetate. The drop in NfL levels also correlated with MRI measures ${ }^{352}$. Daclizumab was also shown to significantly decrease the number of new contrast-enhancing lesions and CSF NfL levels ${ }^{353}$. In contrast to the previously mentioned DMTs, treatment with ibudilast had no significant effect on the baseline and endpoint CSF and serum NfL levels between individuals receiving therapy and placebo $^{354}$. Similarly, no evidence of therapeutic effect concerning NfL concentration was observed in SPMS patients receiving simvastatin or placebo ${ }^{324}$. 
A longitudinal follow-up study described an inverse dose-dependent association between serum $25(\mathrm{OH}) \mathrm{D}$ vitamin and CSF NfL levels ${ }^{327}$. Consequently, a few studies aimed to examine the outcome of vitamin D supplementation in MS. A randomised clinical trial investigating the effect of vitamin-D compared to placebo found no difference in serum NfL levels between the two groups at the end of follow-up ${ }^{319,320}$. A similar study examining 40 interferon treated RRMS patients receiving high dose vitamin-D or placebo established no significant difference between groups at the end of follow-up. Baseline plasma NfL levels were already low, probably due to interferon treatment. At the end of the follow-up, no significant decrease was observed between the placebo and high dose vitamin D groups ${ }^{355}$. However, the potential effect of vitamin D on NfL levels might have been masked by interferon therapy.

The studies mentioned above overwhelmingly indicate that changes in NfL levels represent and correlate well with therapeutic response. However, there is limited evidence on implicating these findings in clinical practice. A recent investigation conducted on 203 patients explored the utility of CSF NfL in therapeutic decision making ${ }^{296}$. Authors reported that NfL levels were particularly useful in progressive MS, where often NfL increase was the only indicator of ongoing disease activity. This indicates that in clinical practice, where clinicians mainly rely on clinical symptoms and MRI measures, insidious presence of disease activity may be missed. Thus NfL might be exceedingly feasible in monitoring therapeutic response in progressive MS. However, in order for NfL measurement to be part of everyday clinical practice, validation studies, age and concomitant disease-related normal ranges and standardisation of laboratory methodologies are mandatory.

Neurofilament heavy chain in MS

Compared to NFL, the role of NfH in MS clinical practise is limited. A study examining CSF NfH levels in CIS compared to non-inflammatory neuropsychiatric disorders revealed significantly elevated $\mathrm{NfH}$ concentration in persons with CIS, which correlated with physical disability and brain volume during the 1-year follow-up, further supporting the presence of axonal damage even in the earliest stages ${ }^{356}$. Similarly to NfL, increased concentration of NfH and tau was a predictor of conversion from CIS to CDMS ${ }^{357}$. Furthermore, in contrast to NfL, only CSF but not serum NfH levels correlated with EDSS scores ${ }^{282}$. Interestingly, despite a higher increase observed in CSF NfH than in NfL levels during disease activity NfL still proved to be a more reliable marker for disease stability ${ }^{340}$. Higher $\mathrm{NfH}$ levels were also associated with relapse activity ${ }^{340,358}$, EDSS $^{359}$ and MSSS $^{360}$ scores and T2 lesion volume ${ }^{360}$. These findings are refuted by other studies which failed to demonstrate a correlation between NfH levels and clinical and MRI variables of MS patients ${ }^{315,324}$. 
Post-mortem examination of brain samples revealed that tissue concentration of hyperphosphorylated NfH shows a strong correlation with NAWM and T1 lesion on post-mortem $\mathrm{MRI}^{361}$. Moreover, serum $\mathrm{NfH}$ concentrations were shown to be moderately associated with T2 lesion volume but not T2 lesion number. Conversely, a correlation with the number of $\mathrm{T} 1$ hypointense lesions but not with $\mathrm{T} 1$ hypointense lesion volume is documented, interestingly $\mathrm{NfH}$ seems to be not related to brain atrophy either ${ }^{282}$. Both CSF NfH and NfL levels were documented to decrease in patients receiving natalizumab however, the reduction observed was more pronounced in NfL levels ${ }^{340}$.

\section{MicroRNA (miRNA)}

MicroRNA (miRNA) is a minuscule, single-stranded, hairpin-like piece of non-coding RNA composed of twenty-some ( 19-24) nucleotides ${ }^{362}$ that is abundantly produced by many cell types. Most of the mapped genes in the human body seem to be targets of miRNAs ${ }^{363,364}$. MiRNAs seem to play a pivotal role in the regulation of several biological processes, which is supported by their exceptional evolutionary conservation $^{365}$. MiRNAs work by regulating gene expression at the posttranscriptional level. They exert their regulatory function by base-pairing with their respective complementary sequence on the targeted mRNA strand. This, in turn, results in the silencing of the affected mRNA molecule by one or more of several processes (i.e. cleavage of the mRNA, decreased translation by ribosomes, loss or shortening of the polyA tail, etc. $)^{366}$. Slightly more than half of the known miRNAs are found within the genes they regulate. Roughly $40 \%$ of the mapped miRNA genes lie in the exons or introns of neighbouring host genes, which they are usually regulated together with ${ }^{367-369}$. In addition to their abundant intracellular expression, miRNAs are readily secreted into the extracellular compartments (ECmiRNA) as well. They can be found circulating in the body fluids such as the CSF and blood ${ }^{370}$, where they are an integral part of the intercellular communication ${ }^{371}$. To date, there is no compelling evidence to determine whether circulating miRNAs are released into the circulation as a cellular byproduct or are specifically released on purpose for a regulatory function. Nonetheless, in contrast to other RNA species, extracellular miRNA molecules are extremely stable ${ }^{372}$. This attribute makes miRNA a perfect candidate for becoming a non-invasive, reproducible and sensitive biomarker for several diseases. Indeed, much attention has been drawn towards miRNAs as biomarkers in the past few years. Not surprisingly, altered miRNA profiles have been discovered in several neurological and autoimmune diseases, including $\mathrm{MS}^{373-376}$.

Unfortunately, there is great heterogeneity among studies evaluating miRNAs' exact role in MS. Most studies have examined the miRNA profiles of peripheral blood mononuclear cells (PBMC) (for an excellent and extensive review see ${ }^{377}$ ) and different subsets of T- and B-cells of MS patients. In contrast, only limited information is 
available on the relationship between ECmiRNAs and the miRNA profile of lesions and normal appearing white matter of MS patients ${ }^{378-381}$. Despite the different tissue types examined, the common observation of the reports is that of a heavily dysregulated miRNA profile in patients, which points toward a global role for miRNAs in MS pathology.

Despite great diversity in MS lesion pathology, the studies examining them have found conserved miRNA profiles clustered around inflammation, gliosis, demyelination and remyelination. Most of the aberrantly expressed miRNAs were shown to regulate resident cells of the CNS implied in MS pathophysiology ${ }^{378-382}$. The miRNA profiling of active lesions has shown dysregulation of several miRNAs (some up-, some downregulated) compared to NAWM. The targets of most (miR-155, miR326, miR-34a, miR-146a, miR-219 and miR-388) of the abnormally regulated miRNAs were found to be responsible for T-cell differentiation, remyelination and macrophage phagocytosis, processes which are known to be prominent in both the development and the resolution os MS lesions ${ }^{378,383}$. An increased amount of miR-326 was reported in both active lesions (most intensely in the Marburg variant of MS) and the peripheral blood of MS patients, particularly during a relapse. The increased production of miR326 enhances Th-17 cell production and reduces the expression of CD47 (an antiphagocytic signal for macrophages). Both these changes have been suggested to play a role in the pathogenesis of $\mathrm{MS}^{378,384}$. Conversely other miRNAs (miR-126-3p, miR-146b-5p, miR-155, miR-196a-5p, miR-21-5p, miR-223-3p, miR-326 and miR-379$5 p$ ) have been shown to be elevated during remission, some of which (miR-223-3p and miR-379) only in men ${ }^{385}$.

In a most recent meta-analysis, Zailaie et al. ${ }^{386}$ aimed to collate research data and summarise the current knowledge of different miRNAs measured in the serum as a diagnostic biomarker for MS. Some miRNAs as a signature or as a panel, while others as a standalone marker were shown to have high enough diagnostic accuracy in differentiating disease forms from each other and distinguishing between healthy controls and MS patients. Of the several hundred miRNAs profiled, a total of 19 were identified to be significantly differentially expressed in MS patients. Eleven were found to be downregulated (miR-145, miR-376 c-3p, miR-128-3p, miR-191-5p, miR26a-5p, miR-320a, miR 486-5p, miR-320b, miR-25-3p, miR-24-3p, miR-140-3p), while 7 (miR-572, miR-15b, miR-23a, let-7 c-5p, miR-16, miR-24, miR-137, miR-181) were shown to be upregulated in the sera of MS patients compared to healthy controls. The expression level of miR-223 (the $19^{\text {th }}$ identified miRNA) was discordant between the reporting studies ${ }^{386}$. The elevation of five miRNAs (miR-572, miR-15b, miR-223, miR128-3p, miR-191-5p) was reliably linked to primary progressive disease. Furthermore, several miRNAs were shown to be nonidentically expressed in different disease subtypes; some (miR-128-3p, miR-191-3p, miR-191-5p, miR-24-3p, miR-26a-5p, miR- 
376 c-3p) were found to be upregulated, while miR-572 was downregulated in the sera of both PP and SPMS patients. MiR-223 and miR-15b were downregulated in PPMS in comparison to SPMS. Conversely, miR-376c-3p was only elevated in PPMS but not in other disease forms. The most dysregulated miRNA in PPMS was found to be circulating miR-191-5p. In contrast, miR-27a-3p was overexpressed in RRMS compared to the progressive forms. Some miRNAs (miR-572, miR-145, miR223, miR137, miR-16, miR-181, miR-24) were established to have better than average sensitivity and specificity to discern between MS patients and HCs, of which the single best miRNA to distinguish between HCs and MS patients was miR-145 with a sensitivity and specificity of $79 \%$ and $87 \%$, respectively ${ }^{386}$.

The target identification of the dysbalanced miRNAs' might explain why the expression of these specific miRNAs is altered in MS. Cytotoxic T-lymphocyteassociated protein 4 (CTLA-4) has been reported to be a target of miR-145 ${ }^{387}$. CTLA-4 is a known inhibitory protein that regulates Treg cell function and enhances the immune system's ability to suppress auto-reactive T-cells, the overactivity of which, among others, have been implicated in the pathogenesis of MS ${ }^{388}$. The exact mechanism by which the aberration of miRNA expression alters the immune system and its connection to autoimmune diseases is controversial and elusive. A plausible scenario in MS is that the observed upregulation of miR-145 silences the CTLA-4 gene, leading to impaired Treg cell function and the increased proliferation of autoreactive T-cells. MiR-191-5p, despite being abundant in the circulation, was found to be downregulated in the NAWM of PPMS patients ${ }^{389,390}$. BDNF, SOX4, FZD5 and WSB1, genes all implicated in CNS homeostasis, were all found to be targets of and reported to show an inverse correlation with the levels of miR-191-5p in NAWM of MS patients. SOX4 is known to promote neural differentiation in the adult CNS. Overexpression of SOX4 has been shown to inhibit oligodendrocyte differentiation from precursor cells, whereas its downregulation results in increased glial maturation ${ }^{391}$. The intense, progressive neurological dysfunction, axonal damage and neurodegeneration seen in the primary progressive phenotype may be, in part, explained by the observed downregulation of miR-191-5p in the NAWM of PPMS patients. This dysbalance might be one of the causes behind the upregulation of SOX4, which in turn results in impaired oligodendrocyte differentiation and, thus, the failure of remyelination and ultimately in the death of neurons. These findings may explain, in part, at least why MS patients' CNS is more susceptible to inflammation and less capable of repair.

The relative sparsity of CNS and immune-system related genes, especially in adolescents proven to be associated with MS, might raise the question that epigenetic changes may play a pivotal role in the pathogenesis of pediatric-onset MS (pedMS). To explore this option, a large scale miRNA genome-wide association study (MIGWAS) and miR-SNP analysis were conducted on 486 pedMS patients and 1362 
control subjects ${ }^{392}$. The MIGWAS method first integrates the results of genome-wide association studies cell- and tissue-type specific expression profiles of miRNAs. Afterwards, it employs miRNA-gene target prediction algorithms to pinpoint the tissue/cell-specific contribution of individual miRNAs to particular diseases and identify miRNAs that might serve as potential biomarkers. With this method, the study has discovered 39 miRNA-target gene pairs consisting of 37 individual genes and 16 distinctive miRNAs. Additionally, it has shown the enrichment of miRNAtarget genes in 25 separate examined tissues without considering for tissue-specific miRNA profiles. Of all the identified tissues, the highest enrichment was found in the keratinised cells of the oral mucosa of the gastrointestinal tract ${ }^{392}$. This is a somewhat expected result as there is a mounting body of evidence of a bidirectional, proinflammatory relationship between $\mathrm{MS}$ and the gut microbiome. The pathologically composed gut microbiome of MS patients was shown to induce a proinflammatory state, and vice versa, the autoreactive immune system of the patient has been observed to shape the gut microbiome ${ }^{393-395}$. The vast majority of the biomarker candidate miRNA-target gene pairs identified by the study are already known to be involved in the activation and signalling apparatus of the immune system. The genes reported are implicated in T-cell activation, class II HLA expression, TGF- $\$$ signalling, proteosome function and degradation targeting, neuronal differentiation and synaptic transmission, also protein folding and homeostasis upkeep of the endoplasmic reticulum (ER). Of the 16 reported miRNAs, five have already been associated with MS; mir-3605 was identified as a candidate biomarker for pedMS by the authors, a result verified by subsequent confirmation studies ${ }^{396,397}$. Interestingly only one, the TVP23B gene, was identified by the miR-SNP analysis to be associated with pedMS. Its exact role in the pathogenesis of MS is unclear, as this gene has been previously linked to diabetic retinopathy, but not MS ${ }^{398}$. Statistical overrepresentation tests conducted on the miR-SNP analysis have shown, however, that a total of 30 genes in 5 crucial signalling pathways are heavily dysbalanced in pedMS patients. The analysis has shown the aberrant regulation of 5 genes in the histamine $\mathrm{H}_{1}$ receptor, 5 genes in the MHC protein complex, 5 genes present on the inner part of the ER lumen, 6 genes the 5- $\mathrm{HT}_{2}$ type receptor-mediated signalling pathway and 9 genes in the interferon-gamma signalling pathways. Furthermore, as expected, pedMS patients were shown to have more copies of the HLADRB1*15:01 allele than controls $^{392}$.

The results of this large scale study strongly suggest that a disbalance in and subsequent faulty regulation by the miRNA system is a significant contributing factor to the development of pediatric MS.

The vast amount of research that in recent years has been directed at the miRNA system and its role in MS pathology has resulted in significant progress. Detailed 
profiles of disease-related cells and tissue types a plethora of aberrantly regulated miRNAs in the plasma, CSF, serum, NAWM and CNS lesions of MS patients have been identified recently, some of which have the potential to become markers for diagnosis and/or progression ${ }^{399-408}$.

As mentioned previously in this section, the binding of a miRNA to its target mRNA causes the degradation of the mRNA molecule in the case of full complementarity or just the inhibition of its translation in the case of incomplete complementarity. Due to redundancy and pleiotropy, the miRNA system is thought to regulate the expression of more than $60 \%$ of the human protein-coding genes ${ }^{363}$. Even though several research groups have identified various miRNAs (more than 700 miRNAs are currently recognised to be dysbalanced in MS) associated with different aspects of MS pathology, there is, unfortunately, significant heterogeneity among the results of the studies. Accordingly, a meta-analysis dealing with the matter has found staggeringly low reproducibility across studies; less than $10 \%$ of the identified miRNAs were found to be imbalanced in the same direction by at least three independent reports ${ }^{407,409}$. Currently, the single, most reliable diagnostic marker for MS is serum miR-145 with a sensitivity close to $90 \%{ }^{407}$. A pair of studies ${ }^{380,410}$ attempted to resolve this problem by trying to link previously reported miRNAs to disease activity characterised by MRI lesion activity. A total of 23 miRNAs were identified this way with consistent expressional changes and a strong correlation to lesion burden and activity. Another large-scale study, large scale MRI based study ${ }^{411}$ has identified three other miRNAs (all playing a role in the upkeep of the blood-brain barrier) as potential differentiators and markers of disease phenotype and progression.

Due to this observed heterogeneity in results, several obstacles have yet to be overcome for miRNAs to become valid, easily comparable and reproducible biomarkers in MS. One of the first challenges is pinpointing the exact members among the severely dysbalanced miRNAs that are not just bystanders but also key players in MS pathogenesis and progression. Another chief problem is the low reproducibility of the studies in the literature. The reason for this is multifactorial. First, miRNA expression is influenced by several factors such as age, disease course, sex, prior or concomitant immunmodulant and steroid treatment. Second, there is significant heterogeneity in the research protocols used, patient selection, the techniques used to isolate and sequence miRNA expression from different kinds of tissues/fluids. Furthermore, various methodologies are used in analysing the gathered data. At the time of writing this article, there is no standardised method for miRNA profiling or data analysis or patient selection to avoid known confounding factors. This needs to be addressed to overcome the current inter-study heterogeneity and low reproducibility issues. Another obstacle is the precise evaluation of miRNA expression in different cell types. Most of the published reports used bulk RNA sequencing to 
measure miRNA levels in the blood (plasma or serum), the CNS or other tissues of MS patients. Therefore, evaluating a specific miRNA's expression level in a single given cell type is extremely difficult. Some research groups have overcome this barrier and have been able to clarify the individual roles of specific miRNAs in peripheral cell types (mostly in PBMCs and lymphocytes). However, accomplishing the same in the resident cells of the CNS (microglia, neurons, oligodendrocytes and astrocytes), which are implicated in MS pathogenesis, lesion activity and repair, remains a challenge ${ }^{377,412}$. In order to step forward in the future, it will be crucial to identify miRNAs that are dysregulated in microglia, neurons, oligodendrocytes and astrocytes, specifically in MS pathology, particularly when it comes to identifying the most effective targets for therapeutic intervention. Should these challenges be overcome, miRNAs have the potential to become extremely useful diagnostic and progression markers; furthermore, they may also give us insight into the pathology of MS as well.

\section{CircularRNA (circRNA)}

Circular RNAs (circRNA) were first described some 45 years ago by Sanger et al. ${ }^{413}$. Still, it was only recently that their regulatory function and biological significance had been confirmed in both physiological and pathological processes. As mentioned in the previous section on miRNAs, the relative failure in the search for the genetic predisposition to MS has shifted the spotlight towards epigenetic factors. This led to the re-discovery of miRNAs and sparked significant interest in their role in autoimmune diseases such as MS ${ }^{401,414-417}$. As the network regulated by miRNAs began to unfold, their redundancy and ability to regulate several different targets was confirmed by several subsequent studies. It became clear that there has to be a yet unknown regulatory mechanism keeping miRNAs in check. Accumulating evidence suggests that one role of circRNAs might be just this; one of their chief functions may be the miRNA system's posttranscriptional regulation.

Similarly to miRNAs, CircRNAs are a species of extremely stable endogenous RNAs found in the blood and other biofluids. Due to the lack of conventional RNA tails, circRNAs are resistant to the RNA endonucleases resulting in their half-life being well over 48 hours $^{418}$. CircRNAs are independently regulated from that of their host genes $^{419}$. They are the products of alternative splicing via four different pathways during which a downstream 5' splice donor site is joined to a 3' upstream splice acceptor site ${ }^{420-422}$. Circular RNAs seem to be a complex group of molecules reflecting their diverse biological role and targets. Based on their origin, three main subtypes have been identified so far; intronic (ciRNA), exonic (ecircRNA) and exon-intron containing circRNAs (EIcircRNA). Most of the known circRNAs are of exonic origin and located in the cytoplasm; meanwhile, ciRNAs are mainly localised in the nucleus and constitute a much smaller fraction of circRNAs. EIcircRNAs are located in the nucleus as well, and they are suggested to play a role in the promotion of 
transcription ${ }^{423,424}$. The circRNA system is highly redundant; a single circRNA can contain more than one binding site for an individual miRNA molecule, concomitantly the same circRNA might bind several different miRNAs simultaneously. Due to this attribute, circRNAs are referred to as intrinsic "sponges" for miRNAs ${ }^{425-427}$. When a circRNA captures a miRNA, the bound miRNA's target messenger RNA is freed from suppression, and transcription may ensue. This way, circRNAs essentially act as posttranslational regulators for the miRNA system. In addition, circRNAs were shown to bind and sequester RNA binding proteins, thus regulating translation and protein production in a miRNA independent manner ${ }^{420}$. Additionally, circRNAs are documented to have impeccable complementarity to linear miRNA species ${ }^{420}$, further expanding their numerous yet unexplored function in biological processes.

Interestingly inverse correlation has been found between a cell type's proliferation rate and circRNA concentration. Rapidly proliferating cell lines are documented to contain less circRNA than cell types with a low proliferation rate ${ }^{428}$. This is in line with the observation of the abundance of circRNAs in the brain compared to other tissues in humans ${ }^{429}$. In recent years circRNAs have been implicated in various immune processes, and immune-mediated disorders also are key players in several diseases affecting the CNS, including $\mathrm{MS}^{430-437}$. A few years ago, the first circRNAs to be dysregulated in MS patients were discovered ${ }^{434,435}$. The identified circRNAs were shown to be connected to various genes (Gasdermin B, MALAT1, IL-7 receptor, SP140) already linked to the pathogenesis of MS. Furthermore, they were also discovered to have a different pattern of expression in relapse and remission and female and male MS patients ${ }^{437-444}$. Using genome-wide association technique ${ }^{445}$, the same group found significant enrichment of non-coding elements in the genomic regions harbouring known MS-associated SNPs. A total of 482 circRNAs were found in the areas of interest vs a mean of $194 \pm 65$ in the random sets. This way, a total of 18 circRNAs were identified (of which 2 were novel) derived from MS-associated genes by the RNA sequencing of two cell lines (SH-SY5Y and Jurkat, both representing tissues relevant for MS). Furthermore, a circRNA (hsa_circ_0043813) from the STAT3 gene (a transcription factor responsible for the polarization of the immune response toward Th17 and thus the development of inflammation) was confirmed to be modulated by three genotypes at the disease-associated SNP. This was later corroborated by the findings of another study, which documented circRNAs affecting the STAT3 pathway to be differently expressed in RRMS patients compared to controls ${ }^{446}$. The downregulation of two additional circRNAs (circ_0005402 and circ_0035560) was reported in MS patients ${ }^{433}$. These circRNAs are located in the ANXA2 gene, which most recently got associated with blood-brain barrier dysfunction in a mouse model of $\mathrm{MS}^{447}$. In addition to circRNAs being implicated in the pathogenesis of MS, they also have been linked to disease activity. Accordingly, three (hsa_circRNA_101348, 
hsa_circRNA_102611, and hsa_circRNA_104361) circRNAs targeting 15 miRNAs and three additional protein-coding RNAs (of which 2 AK2 and IKZF3 are known to be involved in B-cell function) were measured to be overexpressed during a relapse ${ }^{448}$. The disease activity related expression of various circRNAs was corroborated by a subsequent study, which additionally has described the sex-dependent increase of circRNA expression in MS, furthermore has validated and proposed six additional circRNAs as potential biomarkers ${ }^{436}$. The circRNA profile of an individual might not just be an excellent biomarker to monitor relapse activity, but based on recent research, it may be used to differentiate between MS subtypes. Iparraguirre et al. ${ }^{449}$ have found that healthy controls and patients with SP and RR MS have fundamentally different circRNA profiles. They also demonstrated that miRNA "sponging" might not be circRNAs primary function in extracellular vesicles and leukocytes despite prior knowledge. Moreover, the expression of some circRNAs (hsa_circ_0000478 and hsa_circ_0116639) were found to correlate with disease severity and the presence of the anti-myelin lipid-specific oligoclonal IgM bands in the CSF of MS patients ${ }^{450}$.

All of the continuously accumulating data suggest that various non-coding RNA species and the so-called "competing endogenous RNA network" (the detailed reporting of which is unfortunately well beyond the scope of this article, for very extensive literature and a review on the matter see ${ }^{451-454}$ ) might contribute to MS pathogenesis via several already known and yet to be discovered pathways and mechanisms. Circular RNAs are prominent members of this family onto whom intense research has been focused in recent years. Owing to the findings of this intensive research, the circRNA profiling of MS patients may become a reliable tool to predict future disease activity and accurately identify disease phenotype at diagnosis. Furthermore, the changes in one's non-coding RNA profile may become a viable method to monitor disease activity in the near future.

\section{Conclusion}

MS is often called the Lupus of neurology due to the heterogeneity of symptoms it can present with. This diversity, however, is also true for the distinctive pathological processes $^{5,455}$ leading to the development of the disease. Due to the debilitating and irreversible damage MS can cause if left unchecked, it is crucial to diagnose it in the early stages and to commence adequate treatment as soon as possible ${ }^{456}$. The current era in MS with rapidly expanding therapeutic options has raised the demand for prognostic and diagnostic biomarkers and accurate, accessible, validated and minimally invasive markers to closely monitor disease activity, disability progression, and therapeutic response. The aim of treating MS patients nowadays is fundamentally different from that seen decades ago when the slowing of progression was the only achievable aspiration. The current objective is to offer patients completely personalised treatment and completely halt the disease, thus preventing any further 
injury and, if possible, revert some of the already sustained damage to the CNS ${ }^{457-463}$. The disease-modifying treatments used at present are immunomodulatory or immunosuppressive in nature. Currently, the only option for monitoring their therapeutic efficacy in everyday practice are clinical (i.e. the presence or absence of relapses or the presence or lack of EDSS progression in progressive disease forms) and/or MRI measures (i.e. new or equivocally enlarging lesions, number of black holes, contrast enhancement and, if available, total brain/grey matter atrophy). The problem with these methods in conventional MS care is their inability to forecast disease activity, and they might not capture subclinical processes ${ }^{185}$, e.g. the development of psychopathological symptoms ${ }^{464}$, cognitive dysfunction ${ }^{465-467}$, attention network deficits $^{468}$ and subtle neurodegeneration. The soluble biomarkers found in the CSF and/or blood included in this review may eliminate this problem. They can provide the physician with invaluable information on the actually and subclinically ongoing inflammation and neurodegeneration in the CNS of MS patients. These biomarkers are of variable nature (cytokines, chemokines, RNA species etc.). On the one hand, they reflect the diversity of the immune system's involvement in MS pathogenesis and disease progression. On the other hand, they can be used to monitor fundamentally different aspects of the disease. Unfortunately, however, several challenges are yet to be overcome with these biomarkers to be reliable pillars of routine MS care. First of all, none of these markers is specific, neither for MS nor for the CNS (except for neurofilaments, which are specific for neural damage). As such other concomitant diseases (e.g. infections, CNS trauma, stroke, inflammatory or non-inflammatory neurological disease) that affect the immune system and/or damage the CNS may iNfLuence the kinetics and concentrations of these markers. Furthermore, the currently used DMTs have different mechanisms of action and molecular targets; therefore, not every drug might influence the same biomarkers and might affect the ones they do influence with different power. Moreover, a given marker's CSF and blood levels by a given DMT might be influenced with different power making an accurate interpretation even harder. Yet another limitation of many of these inflammatory markers is their sensitivity to degradation by various proteases; therefore, the samples' adequate and rapid pre-analytical handling and storage is crucial for getting valid and reproducible results. However, the biggest hurdle is that even though there is rapidly accumulating data regarding each respective biomarker included in this review, unfortunately, most of the evidence is based on studies of limited size, the results of which are sometimes contradictory. Hopefully, this will change shortly, as owing to recent advances in analytical technology, biomarkers that were previously only detectable in the CSF have now become measurable in blood. This makes repeat lumbar puncture futile and allows for repeat sampling and the less invasive surveillance of MS, thus making the recruitment of bigger study cohorts much simpler. A prime example of this transition from CSF to blood is the case of NFL, 
which, thanks to the development of SiMoA, has become the prime focus of biomarker research in MS, so much so that it is included in more and more RCTs as a non-primary endpoint.

Based on current trends, the extraordinarily diverse and intertwined processes that constitute the pathophysiology of MS and available research data, it is doubtful that a „holy grail” of biomarkers exists for MS. A much more plausible scenario is that the biomarkers above combined as a panel and/or a patient's repeated biomarker profiling is going to be a specific and sensitive enough measure to complement the currently used clinical and MRI parameters in monitoring disease activity, planning therapeutical decisions and truly making medicine more personalised. Despite significant advances in the past couple of years, there is still much to do for this to become a reality. Further studies with larger cohorts and more uniform methodology are needed to validate current and new biomarkers to become solid enough to be incorporated into diagnostic criteria and become widespread enough to become part of routine MS care. Indeed, the next few years in biomarker research will be extremely exciting as it is an exceptionally vibrant scientific area and great breakthroughs are much awaited.

\section{Author Contributions}

Conceptualization, writing-original draft preparation, T.B. and Zs. K.; writingreview and editing, all authors.; supervision, K.B. and L.V. All authors read and approved the final manuscript.

\section{Funding}

This paper was supported by the MTA-SZTE Neuroscience Research Group and OTKA138-125-K grants.

\section{Data Availability Statement}

Not applicable.

\section{Conflicts of Interest}

The authors report no conflict of interest

\section{References}

1. Magyari M, Sorensen PS. The changing course of multiple sclerosis: rising incidence, change in geographic distribution, disease course, and prognosis. Curr Opin Neurol. 2019;32(3). doi:10.1097/WCO.0000000000000695

2. Biernacki T, Sandi D, Fricska-Nagy Z, et al. Epidemiology of multiple sclerosis in Central Europe, update from Hungary. Brain Behav. 2020;10(5). doi:10.1002/BRB3.1598

3. Koch-Henriksen N, Magyari M. Apparent changes in the epidemiology and severity of multiple sclerosis. Nat Rev Neurol. 2021;17(11):676-688. doi:10.1038/S41582-021-00556-Y

4. Lassmann H. Multiple Sclerosis Pathology. Cold Spring Harb Perspect Med. 2018;8(3). doi:10.1101/CSHPERSPECT.A028936 
5. Lassmann H. Pathology and disease mechanisms in different stages of multiple sclerosis. J Neurol Sci. 2013;333(1-2):1-4. doi:10.1016/J.JNS.2013.05.010

6. Miller DH, Chard DT, Ciccarelli O. Clinically isolated syndromes. Lancet Neurol. 2012;11(2):157-169. doi:10.1016/S1474-4422(11)70274-5

7. Kuhle J, Disanto G, Dobson R, et al. Conversion from clinically isolated syndrome to multiple sclerosis: A large multicantre study. Mult Scler. 2015;21(8):1013-1024. doi:10.1177/1352458514568827

8. Thompson AJ, Banwell BL, Barkhof F, et al. Diagnosis of multiple sclerosis: 2017 revisions of the McDonald criteria. Lancet Neurol. 2018;17(2):162-173. doi:10.1016/S1474-4422(17)30470-2

9. Gaetani L, Fanelli F, Riccucci I, et al. High risk of early conversion to multiple sclerosis in clinically isolated syndromes with dissemination in space at baseline. J Neurol Sci. 2017;379:236-240. doi:10.1016/J.JNS.2017.06.008

10. Marcus JF, Waubant EL. Updates on clinically isolated syndrome and diagnostic criteria for multiple sclerosis. The Neurohospitalist. 2013;3(2):65-80. doi:10.1177/1941874412457183

11. Efendi H. Clinically Isolated Syndromes: Clinical Characteristics, Differential Diagnosis, and Management. Noro Psikiyatr Ars. 2015;52(Suppl 1):S1-S11. doi:10.5152/NPA.2015.12608

12. Dobson R, Giovannoni G. Multiple sclerosis - a review. Eur J Neurol. 2019;26(1):27-40. doi:10.1111/ENE.13819

13. Bjornevik K, Cortese M, Healy BC, et al. Longitudinal analysis reveals high prevalence of Epstein-Barr virus associated with multiple sclerosis. Science (80- ). 2022;375(6578):296-301. doi:10.1126/SCIENCE.ABJ8222/SUPPL_FILE/SCIENCE.ABJ8222_MDAR_REPRODUCIBILITY_CHECKLI ST.PDF

14. Ruiz F, Vigne S, Pot C. Resolution of inflammation during multiple sclerosis. Semin Immunopathol. 2019;41(6):711-726. doi:10.1007/S00281-019-00765-0

15. Confavreux C, Vukusic S. Natural history of multiple sclerosis: a unifying concept. Brain. 2006;129(Pt 3):606-616. doi:10.1093/BRAIN/AWL007

16. Scalfari A, Neuhaus A, Degenhardt A, et al. The natural history of multiple sclerosis, a geographically based study 10: relapses and long-term disability. Brain. 2010;133(7). doi:10.1093/brain/awq118

17. Kurtzke JF. Rating neurologic impairment in multiple sclerosis: An expanded disability status scale (EDSS). Neurology. 1983;33(11). doi:10.1212/WNL.33.11.1444

18. Filippini G, Del Giovane C, Vacchi L, et al. Immunomodulators and immunosuppressants for multiple sclerosis: a network meta-analysis. Cochrane database Syst Rev. 2013;2013(6). doi:10.1002/14651858.CD008933.PUB2

19. Pozzilli C, Prosperini L. Clinical markers of therapeutic response to disease modifying drugs. Neurol Sci. 2008;29 Suppl 2(SUPPL. 2). doi:10.1007/S10072-008-0939-9

20. Wattjes MP, Ciccarelli O, Reich DS, et al. 2021 MAGNIMS-CMSC-NAIMS consensus recommendations on the use of MRI in patients with multiple sclerosis. Lancet Neurol. 2021;20(8):653-670. doi:10.1016/S14744422(21)00095-8

21. Kappos L, Wolinsky JS, Giovannoni G, et al. Contribution of Relapse-Independent Progression vs Relapse-Associated Worsening to Overall Confirmed Disability Accumulation in Typical Relapsing Multiple Sclerosis in a Pooled Analysis of 2 Randomized Clinical Trials. JAMA Neurol. 2020;77(9):11321140. doi:10.1001/jamaneurol.2020.1568

22. Kalincik T, Cutter G, Spelman T, et al. Defining reliable disability outcomes in multiple sclerosis. Brain. 2015;138(Pt 11):3287-3298. doi:10.1093/BRAIN/AWV258

23. Noseworthy JH, Vandervoort MK, Wong CJ, Ebers GC. Interrater variability with the Expanded Disability Status Scale (EDSS) and Functional Systems (FS) in a multiple sclerosis clinical trial. Neurology. 
1990;40(6):971-971. doi:10.1212/WNL.40.6.971

24. Isaacs A, Baker M, Wavrant-De Vrièze F, Hutton M. Determination of the gene structure of human GFAP and absence of coding region mutations associated with frontotemporal dementia with parkinsonism linked to chromosome 17. Genomics. 1998;51(1):152-154. doi:10.1006/GENO.1998.5360

25. Human hg38 chr17:44903161-44916937 UCSC Genome Browser v426. Accessed February 13, 2022. https://genome.ucsc.edu/cgi-

bin/hgTracks?db=hg38\&lastVirtModeType=default\&lastVirtModeExtraState=\&virtModeType=default\&vi rtMode=0\&nonVirtPosition=\&position=chr17\%3A4490316144916937\&hgsid=1282455267_z3AHFf83XyJxvLjdaYaDeGZLVQwm

26. Cullen DK, Simon CM, LaPlaca MC. Strain rate-dependent induction of reactive astrogliosis and cell death in three-dimensional neuronal-astrocytic co-cultures. Brain Res. 2007;1158(1):103-115. doi:10.1016/J.BRAINRES.2007.04.070

27. Eng LF, Ghirnikar RS, Lee YL. Glial Fibrillary Acidic Protein: GFAP-Thirty-One Years (1969-2000). Neurochem Res 2000 259. 2000;25(9):1439-1451. doi:10.1023/A:1007677003387

28. Abdelhak A, Huss A, Kassubek J, Tumani H, Otto M. Serum GFAP as a biomarker for disease severity in multiple sclerosis. Sci Reports 2018 81. 2018;8(1):1-7. doi:10.1038/s41598-018-33158-8

29. Axelsson M, Malmeström C, Nilsson S, Haghighi S, Rosengren L, Lycke J. Glial fibrillary acidic protein: a potential biomarker for progression in multiple sclerosis. J Neurol 2010 2585. 2011;258(5):882-888. doi:10.1007/S00415-010-5863-2

30. Haghighi S, Andersen O, Odén A, Rosengren L. Cerebrospinal fluid markers in MS patients and their healthy siblings. Acta Neurol Scand. 2004;109(2):97-99. doi:10.1034/J.1600-0404.2003.00197.X

31. Ayrignac X, Le Bars E, Duflos C, et al. Serum GFAP in multiple sclerosis: correlation with disease type and MRI markers of disease severity. Sci Reports 2020 101. 2020;10(1):1-5. doi:10.1038/s41598-020-67934-2

32. Huss A, Otto M, Senel M, Ludolph AC, Abdelhak A, Tumani H. A Score Based on NfL and Glial Markers May Differentiate Between Relapsing-Remitting and Progressive MS Course. Front Neurol. 2020;11:608. doi:10.3389/FNEUR.2020.00608/BIBTEX

33. Norgren N, Sundström P, Svenningsson A, Rosengren L, Stigbrand T, Gunnarsson M. Neurofilament and glial fibrillary acidic protein in multiple sclerosis. Neurology. 2004;63(9):1586-1590. doi:10.1212/01.WNL.0000142988.49341.D1

34. Mañé-Martínez MA, Olsson B, Bau L, et al. Glial and neuronal markers in cerebrospinal fluid in different types of multiple sclerosis. J Neuroimmunol. 2016;299:112-117. doi:10.1016/J.JNEUROIM.2016.08.004

35. Rosengren LE, Lycke J, Andersen O. Glial fibrillary acidic protein in CSF of multiple sclerosis patients: relation to neurological deficit. J Neurol Sci. 1995;133(1):61-65. doi:10.1016/0022-510X(95)00152-R

36. Petzold A, Eikelenboom MJ, Gveric D, et al. Markers for different glial cell responses in multiple sclerosis: clinical and pathological correlations. Brain. 2002;125(7):1462-1473. doi:10.1093/BRAIN/AWF165

37. Sun MJ, Liu N, Xie QF, et al. A candidate biomarker of glial fibrillary acidic protein in CSF and blood in differentiating multiple sclerosis and its subtypes: A systematic review and meta-analysis. Mult Scler Relat Disord. 2021;51. doi:10.1016/J.MSARD.2021.102870

38. Axelsson M, Malmeström C, Gunnarsson M, et al. Immunosuppressive therapy reduces axonal damage in progressive multiple sclerosis. Mult Scler. 2014;20(1):43-50. doi:10.1177/1352458513490544

39. Gunnarsson M, Malmeström C, Axelsson M, et al. Axonal damage in relapsing multiple sclerosis is markedly reduced by natalizumab. Ann Neurol. 2011;69(1):83-89. doi:10.1002/ANA.22247

40. Abdelhak A, Hottenrott T, Morenas-Rodríguez E, et al. Glial Activation Markers in CSF and Serum From Patients With Primary Progressive Multiple Sclerosis: Potential of Serum GFAP as Disease Severity Marker? Front Neurol. 2019;10:280. doi:10.3389/FNEUR.2019.00280/BIBTEX 
41. Högel H, Rissanen E, Barro C, et al. Serum glial fibrillary acidic protein correlates with multiple sclerosis disease severity. Mult Scler J. 2020;26(2):210-219. doi:10.1177/1352458518819380

42. Xie XF, Huang XH, Shen AZ, Li J, Sun YH. Association between circulating leptin levels and multiple sclerosis: a systematic review and meta-analysis. Postgrad Med J. 2018;94(1111):278-283. doi:10.1136/POSTGRADMEDJ-2017-135397

43. Conde J, Scotece M, Gómez R, Gómez-Reino JJ, Lago F, Gualillo O. At the crossroad between immunity and metabolism: focus on leptin. $h t t p: / / d x$.doi.org/101586/eci1048. 2014;6(5):801-808. doi:10.1586/ECI.10.48

44. Green ED, Maffei M, Braden V V., et al. The human obese (OB) gene: RNA expression pattern and mapping on the physical, cytogenetic, and genetic maps of chromosome 7. Genome Res. 1995;5(1):5-12. doi:10.1101/GR.5.1.5

45. Cirillo D, Rachiglio AM, La Montagna R, Giordano A, Normanno N. Leptin signaling in breast cancer: An overview. J Cell Biochem. 2008;105(4):956-964. doi:10.1002/JCB.21911

46. Auwerx J, Staels B. Leptin. Lancet. 1998;351(9104):737-742. doi:10.1016/S0140-6736(97)06348-4

47. Konstantinides S, Schäfer K, Loskutoff DJ. The Prothrombotic Effects of Leptin. Ann N Y Acad Sci. 2001;947(1):134-142. doi:10.1111/J.1749-6632.2001.TB03936.X

48. Fujita Y. Leptin and autoimmune disease. Nihon Rinsho Meneki Gakkai Kaishi. 2017;40(3):155-159. doi:10.2177/JSCI.40.155

49. Huitema MJD, Schenk GJ. Insights into the Mechanisms That May Clarify Obesity as a Risk Factor for Multiple Sclerosis. Curr Neurol Neurosci Rep. 2018;18(4):1-9. doi:10.1007/S11910-018-0827-5/FIGURES/1

50. Härle P, Straub RH. Leptin Is a Link between Adipose Tissue and Inflammation. Ann N Y Acad Sci. 2006;1069(1):454-462. doi:10.1196/ANNALS.1351.044

51. Marrodan M, Farez MF, Balbuena Aguirre ME, Correale J. Obesity and the risk of Multiple Sclerosis. The role of Leptin. Ann Clin Transl Neurol. 2021;8(2):406-424. doi:10.1002/ACN3.51291

52. La Cava A, Matarese G. The weight of leptin in immunity. Nat Rev Immunol 2004 45. 2004;4(5):371-379. doi:10.1038/nri1350

53. Rodríguez-Sáinz M del C, Sánchez-Ramón S, Andrés C de, Rodríguez-Mahou M, Muñoz-Fernández MA. Th1/Th2 cytokine balance and nitric oxide in cerebrospinal fluid and serum from patients with multiple sclerosis. Eur Cytokine Netw. 2002;13(1):110-114. Accessed February 10, 2022.

http://www.jle.com/fr/revues/ecn/e-

docs/th1_th2_cytokine_balance_and_nitric_oxide_in_cerebrospinal_fluid_and_serum_from_patients_wit h_multiple_sclerosis_90048/article.phtml?tab=texte

54. Oreja-Guevara C, Ramos-Cejudo J, Aroeira LS, Chamorro B, Diez-Tejedor E. TH1/TH2 Cytokine profile in relapsing-remitting multiple sclerosis patients treated with Glatiramer acetate or Natalizumab. BMC Neurol. 2012;12(1):1-6. doi:10.1186/1471-2377-12-95/FIGURES/2

55. Tumani H, Kassubek J, Hijazi M, et al. Patterns of Th1/Th2 Cytokines Predict Clinical Response in Multiple Sclerosis Patients Treated with Glatiramer Acetate. Eur Neurol. 2011;65(3):164-169. doi:10.1159/000324035

56. Messina S, Vargas-Lowy D, Musallam A, et al. Increased leptin and A-FABP levels in relapsing and progressive forms of MS. BMC Neurol. 2013;13(1):1-8. doi:10.1186/1471-2377-13-172/TABLES/4

57. Biström M, Hultdin J, Andersen O, et al. Leptin levels are associated with multiple sclerosis risk. Mult Scler. 2021;27(1):19-27. doi:10.1177/1352458520905033

58. Rotondi M, Batocchi AP, Coperchini F, et al. Severe Disability in Patients with Relapsing-Remitting Multiple Sclerosis Is Associated with Profound Changes in the Regulation of Leptin Secretion. Neuroimmunomodulation. 2013;20(6):341-347. doi:10.1159/000353567

59. Batocchi AP, Rotondi M, Caggiula M, et al. Leptin as a marker of multiple sclerosis activity in patients 
treated with interferon-beta. J Neuroimmunol. 2003;139(1):150-154. doi:10.1016/S0165-5728(03)00154-1

60. Chatzantoni K, Papathanassopoulos P, Gourzoulidou E, Mouzaki A. Leptin and its soluble receptor in plasma of patients suffering from remitting-relapsing multiple sclerosis (MS): In vitro effects of leptin on type- 1 and type- 2 cytokine secretion by peripheral blood mononuclear cells, T-cells and monocytes of MS patients. J Autoimmun. 2004;23(2):169-177. doi:10.1016/J.JAUT.2004.05.007

61. Emamgholipour S, Eshaghi SM, Hossein-nezhad A, Mirzaei K, Maghbooli Z, Sahraian MA. Correction: Adipocytokine Profile, Cytokine Levels and Foxp3 Expression in Multiple Sclerosis: a Possible Link to Susceptibility and Clinical Course of Disease. PLoS One. 2013;8(11):e76555. doi:10.1371/ANNOTATION/6DE84C11-73F5-4EE4-AEC7-CA83072401A4

62. Kraszula Ł, Jasińska A, Eusebio MO, Kuna P, Głabiński A, Pietruczuk M. Evaluation of the relationship between leptin, resistin, adiponectin and natural regulatory T cells in relapsing-remitting multiple sclerosis. Neurol Neurochir Pol. 2012;46(1):22-28. doi:10.5114/NINP.2012.27211

63. Dashti M, Alroughani R, Jacob S, Al-Temaimi R. Leptin rs7799039 polymorphism is associated with multiple sclerosis risk in Kuwait. Mult Scler Relat Disord. 2019;36. doi:10.1016/J.MSARD.2019.101409

64. Maisonpierre PC, Le Beau MM, Espinosa R, et al. Human and rat brain-derived neurotrophic factor and neurotrophin-3: gene structures, distributions, and chromosomal localizations. Genomics. 1991;10(3):558568. doi:10.1016/0888-7543(91)90436-I

65. Pruunsild P, Kazantseval A, Aid T, Palm K, Timmusk T. Dissecting the human BDNF locus: bidirectional transcription, complex splicing, and multiple promoters. Genomics. 2007;90(3):397-406. doi:10.1016/J.YGENO.2007.05.004

66. Baj G, Tongiorgi E. BDNF splice variants from the second promoter cluster support cell survival of differentiated neuroblastoma upon cytotoxic stress. J Cell Sci. 2009;122(Pt 1):36-43. doi:10.1242/JCS.033316

67. PC M, L B, S S, et al. Neurotrophin-3: a neurotrophic factor related to NGF and BDNF. Science. 1990;247(4949 Pt 1):1446-1451. doi:10.1126/SCIENCE.247.4949.1446

68. Allen SJ, Watson JJ, Shoemark DK, Barua NU, Patel NK. GDNF, NGF and BDNF as therapeutic options for neurodegeneration. Pharmacol Ther. 2013;138(2):155-175. doi:10.1016/J.PHARMTHERA.2013.01.004

69. K B. Neurotrophins and their receptors in early development of the mammalian nervous system. Acta Neurobiol Exp (Wars). 2010;70(4):454-467.

70. Bliss TVP, Collingridge GL. A synaptic model of memory: long-term potentiation in the hippocampus. Nat 1993 3616407. 1993;361(6407):31-39. doi:10.1038/361031a0

71. Figurov A, Pozzo-Miller LD, Olafsson P, Wang T, Lu B. Regulation of synaptic responses to highfrequency stimulation and LTP by neurotrophins in the hippocampus. Nature. 1996;381(6584):706-709. doi:10.1038/381706A0

72. Korte M, Carroll P, Wolf E, Brem G, Thoenen H, Bonhoeffer T. Hippocampal long-term potentiation is impaired in mice lacking brain-derived neurotrophic factor. Proc Natl Acad Sci U S A. 1995;92(19):8856. doi:10.1073/PNAS.92.19.8856

73. Liepinsh E, Ilag LL, Otting G, Ibáñez CF. NMR structure of the death domain of the p75 neurotrophin receptor. EMBO J. 1997;16(16):4999-5005. doi:10.1093/EMBOJ/16.16.4999

74. Egan MF, Kojima M, Callicott JH, et al. The BDNF val66met polymorphism affects activity-dependent secretion of BDNF and human memory and hippocampal function. Cell. 2003;112(2):257-269. doi:10.1016/S0092-8674(03)00035-7

75. Petryshen TL, Sabeti PC, Aldinger KA, et al. Population genetic study of the brain-derived neurotrophic factor (BDNF) gene. Mol Psychiatry. 2010;15(8):810-815. doi:10.1038/MP.2009.24

76. Pivac N, Byungsu Kim, Gordana Nedić, et al. Ethnic differences in brain-derived neurotrophic factor Val66Met polymorphism in Croatian and Korean healthy participants. Croat Med J. 2009;50(1):43-48. doi:10.3325/CMJ.2009.50.43 
77. Zhang H, Ozbay F, Lappalainen J, et al. Brain derived neurotrophic factor (BDNF) gene variants and Alzheimer's disease, affective disorders, posttraumatic stress disorder, schizophrenia, and substance dependence. Am J Med Genet B Neuropsychiatr Genet. 2006;141B(4):387-393. doi:10.1002/AJMG.B.30332

78. Ventriglia M, Chiavetto LB, Benussi L, et al. Association between the BDNF 196 A/G polymorphism and sporadic Alzheimer's disease. Mol Psychiatry. 2002;7(2):136-137. doi:10.1038/SJ.MP.4000952

79. Shimizu E, Hashimoto K, Iyo M. Ethnic difference of the BDNF 196G/A (val66met) polymorphism frequencies: the possibility to explain ethnic mental traits. Am J Med Genet B Neuropsychiatr Genet. 2004;126B(1):122-123. doi:10.1002/AJMG.B.20118

80. Bian JT, Zhang JW, Zhang ZX, Zhao HL. Association analysis of brain-derived neurotrophic factor (BDNF) gene 196 A/G polymorphism with Alzheimer's disease (AD) in mainland Chinese. Neurosci Lett. 2005;387(1):11-16. doi:10.1016/J.NEULET.2005.07.009

81. Olin D, MacMurray J, Comings DE. Risk of late-onset Alzheimer's disease associated with BDNF C270T polymorphism. Neurosci Lett. 2005;381(3):275-278. doi:10.1016/J.NEULET.2005.02.017

82. Mirowska-Guzel D, Mach A, Gromadzka G, Czlonkowski A, Czlonkowska A. BDNF A196G and C270T gene polymorphisms and susceptibility to multiple sclerosis in the Polish population. Gender differences. J Neuroimmunol. 2008;193(1-2):170-172. doi:10.1016/J.JNEUROIM.2007.10.013

83. Weinstock-Guttman B, Benedict RHB, Tamaño-Blanco M, et al. The rs2030324 SNP of brain-derived neurotrophic factor (BDNF) is associated with visual cognitive processing in multiple sclerosis. Pathophysiol Off J Int Soc Pathophysiol. 2011;18(1):43-52. doi:10.1016/J.PATHOPHYS.2010.04.005

84. Bodner SM, Berrettini W, Van Deerlin V, et al. Genetic variation in the brain derived neurotrophic factor gene in Alzheimer's disease. Am J Med Genet B Neuropsychiatr Genet. 2005;134B(1):1-5. doi:10.1002/AJMG.B.30154

85. Fukumoto N, Fujii T, Combarros O, et al. Sexually dimorphic effect of the Val66Met polymorphism of BDNF on susceptibility to Alzheimer's disease: New data and meta-analysis. Am J Med Genet B Neuropsychiatr Genet. 2010;153B(1):235-242. doi:10.1002/AJMG.B.30986

86. Vepsäläinen S, Castren E, Helisalmi S, et al. Genetic analysis of BDNF and TrkB gene polymorphisms in Alzheimer's disease. J Neurol. 2005;252(4):423-428. doi:10.1007/S00415-005-0667-5

87. Matsushita S, Arai H, Matsui T, et al. Brain-derived neurotrophic factor gene polymorphisms and Alzheimer's disease. J Neural Transm. 2005;112(5):703-711. doi:10.1007/S00702-004-0210-3

88. Mero IL, Smestad C, Lie BA, et al. Polymorphisms of the BDNF gene show neither association with multiple sclerosis susceptibility nor clinical course. J Neuroimmunol. 2012;244(1-2):107-110. doi:10.1016/J.JNEUROIM.2012.01.011

89. Liguori M, Fera F, Gioia MC, et al. Investigating the role of brain-derived neurotrophic factor in relapsing-remitting multiple sclerosis. Genes Brain Behav. 2007;6(2):177-183. doi:10.1111/J.1601183X.2006.00245.X

90. Ramasamy DP, Ramanathan M, Cox JL, et al. Effect of Met66 allele of the BDNF rs6265 SNP on regional gray matter volumes in patients with multiple sclerosis: A voxel-based morphometry study. Pathophysiol Off J Int Soc Pathophysiol. 2011;18(1):53-60. doi:10.1016/J.PATHOPHYS.2010.04.006

91. Zivadinov R, Weinstock-Guttman B, Benedict R, et al. Preservation of gray matter volume in multiple sclerosis patients with the Met allele of the rs6265 (Val66Met) SNP of brain-derived neurotrophic factor. Hum Mol Genet. 2007;16(22):2659-2668. doi:10.1093/HMG/DDM189

92. Fera F, Passamonti L, Cerasa A, et al. The BDNF Val66Met Polymorphism Has Opposite Effects on Memory Circuits of Multiple Sclerosis Patients and Controls. PLoS One. 2013;8(4):e61063. doi:10.1371/JOURNAL.PONE.0061063

93. Portaccio E, Bellinvia A, Prestipino E, et al. The Brain-Derived Neurotrophic Factor Val66Met Polymorphism Can Protect Against Cognitive Impairment in Multiple Sclerosis. Front Neurol. 2021;12:236. doi:10.3389/FNEUR.2021.645220/BIBTEX 
94. Naegelin Y, Saeuberli K, Schaedelin S, et al. Levels of brain-derived neurotrophic factor in patients with multiple sclerosis. Ann Clin Transl Neurol. 2020;7(11):2251-2261. doi:10.1002/ACN3.51215

95. Lindquist S, Schott BH, Ban M, Compston DAS, Sawcer S, Sailer M. The BDNF-Val66Met polymorphism: implications for susceptibility to multiple sclerosis and severity of disease. J Neuroimmunol. 2005;167(12):183-185. doi:10.1016/J.JNEUROIM.2005.06.008

96. Blanco Y, Gómez-Choco M, Arostegui JL, et al. No association of the Val66Met polymorphism of brainderived neurotrophic factor (BDNF) to multiple sclerosis. Neurosci Lett. 2006;396(3):217-219. doi:10.1016/J.NEULET.2005.11.032

97. Nociti V, Santoro M, Quaranta D, et al. BDNF rs6265 polymorphism methylation in Multiple Sclerosis: A possible marker of disease progression. PLoS One. 2018;13(10):e0206140. doi:10.1371/JOURNAL.PONE.0206140

98. Stadelmann C, Kerschensteiner M, Misgeld T, Brück W, Hohlfeld R, Lassmann H. BDNF and gp145trkB in multiple sclerosis brain lesions: neuroprotective interactions between immune and neuronal cells? Brain. 2002;125(1):75-85. doi:10.1093/BRAIN/AWF015

99. Steinman L. Multiple sclerosis: a two-stage disease. Nat Immunol 2001 29. 2001;2(9):762-764. doi:10.1038/ni0901-762

100. Noseworthy JH, Lucchinetti C, Rodriguez M, Weinshenker BG. Multiple sclerosis. N Engl J Med. 2000;343(13):938-952. doi:10.1056/NEJM200009283431307

101. Bjartmar C, Trapp BD. Axonal and neuronal degeneration in multiple sclerosis: mechanisms and functional consequences. Curr Opin Neurol. 2001;14(3):271-278. doi:10.1097/00019052-200106000-00003

102. Tonra JR, Curtis R, Wong V, et al. Axotomy Upregulates the Anterograde Transport and Expression of Brain-Derived Neurotrophic Factor by Sensory Neurons. J Neurosci. 1998;18(11):4374. doi:10.1523/JNEUROSCI.18-11-04374.1998

103. Trapp BD, Peterson J, Ransohoff RM, Rudick R, Mörk S, Bö L. Axonal transection in the lesions of multiple sclerosis. N Engl J Med. 1998;338(5):278-285. doi:10.1056/NEJM199801293380502

104. Caggiula M, Batocchi AP, Frisullo G, et al. Neurotrophic factors and clinical recovery in relapsingremitting multiple sclerosis. Scand J Immunol. 2005;62(2):176-182. doi:10.1111/J.1365-3083.2005.01649.X

105. Sarchielli P, Greco L, Stipa A, Floridi A, Gallai V. Brain-derived neurotrophic factor in patients with multiple sclerosis. J Neuroimmunol. 2002;132(1-2):180-188. doi:10.1016/S0165-5728(02)00319-3

106. Azoulay D, Urshansky N, Karni A. Low and dysregulated BDNF secretion from immune cells of MS patients is related to reduced neuroprotection. J Neuroimmunol. 2008;195(1-2):186-193. doi:10.1016/J.JNEUROIM.2008.01.010

107. Azoulay D, Vachapova V, Shihman B, Miler A, Karni A. Lower brain-derived neurotrophic factor in serum of relapsing remitting MS: reversal by glatiramer acetate. J Neuroimmunol. 2005;167(1-2):215-218. doi:10.1016/J.JNEUROIM.2005.07.001

108. Hamamcioglu K, Reder AT. Interferon- $\beta$ regulates cytokines and BDNF: Greater effect in relapsing than in progressive multiple sclerosis. Mult Scler. 2007;13(4):459-470. doi:10.1177/1352458506069672

109. Land H, Schütz G, Schmale H, Richter D. Nucleotide sequence of cloned cDNA encoding bovine arginine vasopressin-neurophysin II precursor. Nature. 1982;295(5847):299-303. doi:10.1038/295299A0

110. Struck J, Morgenthaler NG, Bergmann A. Copeptin, a stable peptide derived from the vasopressin precursor, is elevated in serum of sepsis patients. Peptides. 2005;26(12):2500-2504. doi:10.1016/J.PEPTIDES.2005.04.019

111. Katan M, Müller B, Christ-Crain M. Copeptin: A new and promising diagnostic and prognostic marker. Crit Care. 2008;12(2):1-2. doi:10.1186/CC6799/METRICS

112. Acher R, Chauvet J, Rouille Y. Dynamic processing of neuropeptides: sequential conformation shaping of 
neurohypophysial preprohormones during intraneuronal secretory transport. J Mol Neurosci. 2002;18(3):223-228. doi:10.1385/JMN:18:3:223

113. Robertson GL, Mahr EA, Athar S, Sinha T. Development and Clinical Application of a New Method for the Radioimmunoassay of Arginine Vasopressin in Human Plasma. J Clin Invest. 1973;52(9):2340-2352. doi:10.1172/JCI107423

114. Preibisz JJ, Sealey JE, Laragh JH, Cody RJ, Weksler BB. Plasma and platelet vasopressin in essential hypertension and congestive heart failure. Hypertens (Dallas, Tex 1979). 1983;5(2 Pt 2):I-129-I-138. doi:10.1161/01.HYP.5.2_PT_2.I129

115. Uchoa ET, Aguilera G, Herman JP, Fiedler JL, Deak T, de Sousa MBC. Novel Aspects of Glucocorticoid Actions. J Neuroendocrinol. 2014;26(9):557-572. doi:10.1111/JNE.12157

116. Katan M, Christ-Crain M. The stress hormone copeptin: a new prognostic biomarker in acute illness. Swiss Med Wkly. 2010;140(NOVEMBER):11-15. doi:10.4414/SMW.2010.13101

117. Boeckel JN, Oppermann J, Anadol R, Fichtlscherer S, Zeiher AM, Keller T. Analyzing the Release of Copeptin from the Heart in Acute Myocardial Infarction Using a Transcoronary Gradient Model. Sci Reports 2016 61. 2016;6(1):1-5. doi:10.1038/srep20812

118. Krismer AC, Wenzel V, Stadlbauer KH, et al. Vasopressin during cardiopulmonary resuscitation: a progress report. Crit Care Med. 2004;32(9 Suppl). doi:10.1097/01.CCM.0000134267.91520.C0

119. Morgenthaler NG, Struck J, Jochberger S, Dünser MW. Copeptin: clinical use of a new biomarker. Trends Endocrinol Metab. 2008;19(2):43-49. doi:10.1016/J.TEM.2007.11.001

120. Gold SM, Raji A, Huitinga I, Wiedemann K, Schulz KH, Heesen C. Hypothalamo-pituitary-adrenal axis activity predicts disease progression in multiple sclerosis. J Neuroimmunol. 2005;165(1-2):186-191. doi:10.1016/J.JNEUROIM.2005.04.014

121. Reder AT, Makowiec RL, Lowy MT. Adrenal size is increased in multiple sclerosis. Arch Neurol. 1994;51(2):151-154. doi:10.1001/ARCHNEUR.1994.00540140057015

122. Erkut ZA, Endert E, Huitinga I, Swaab DF. Cortisol is increased in postmortem cerebrospinal fluid of multiple sclerosis patients: relationship with cytokines and sepsis. Mult Scler. 2002;8(3):229-236. doi:10.1191/1352458502MS797OA

123. Huitinga I, Erkut ZA, Van Beurden D, Swaab DF. Impaired hypothalamus-pituitary-adrenal axis activity and more severe multiple sclerosis with hypothalamic lesions. Ann Neurol. 2004;55(1):37-45. doi:10.1002/ANA.10766

124. Kern S, Krause I, Horntrich A, Thomas K, Aderhold J, Ziemssen T. Cortisol Awakening Response Is Linked to Disease Course and Progression in Multiple Sclerosis. PLoS One. 2013;8(4):e60647. doi:10.1371/JOURNAL.PONE.0060647

125. Dobša L, Cullen Edozien K. Copeptin and its potential role in diagnosis and prognosis of various diseases. Biochem medica. 2013;23(2):172-190. doi:10.11613/BM.2013.021

126. Katan M, Fluri F, Morgenthaler NG, et al. Copeptin: a novel, independent prognostic marker in patients with ischemic stroke. Ann Neurol. 2009;66(6):799-808. doi:10.1002/ANA.21783

127. Koseoglu M, Ozben S, Gozubatik-Celik G, et al. Plasma copeptin levels in patients with multiple sclerosis. J Clin Neurosci. 2020;78:143-146. doi:10.1016/J.JOCN.2020.04.095

128. Baranowska-Bik A, Kochanowski J, Uchman D, et al. Association of copeptin and cortisol in newly diagnosed multiple sclerosis patients. J Neuroimmunol. 2015;282:21-24. doi:10.1016/J.JNEUROIM.2015.03.011

129. Prokopova B, Hlavacova N, Vlcek M, et al. Early cognitive impairment along with decreased stressinduced BDNF in male and female patients with newly diagnosed multiple sclerosis. J Neuroimmunol. 2017;302:34-40. doi:10.1016/J.JNEUROIM.2016.11.007 
130. Zhao T, Su Z, Li Y, Zhang X, You Q. Chitinase-3 like-protein-1 function and its role in diseases. Signal Transduct Target Ther 2020 51. 2020;5(1):1-20. doi:10.1038/s41392-020-00303-7

131. Bonneh-Barkay D, Wang G, Starkey A, Hamilton RL, Wiley CA. In vivo CHI3L1 (YKL-40) expression in astrocytes in acute and chronic neurological diseases. J Neuroinflammation. 2010;7(1):1-8. doi:10.1186/17422094-7-34/FIGURES/5

132. CHI3L1 chitinase 3 like 1 [ Homo sapiens (human) ]. https://www.ncbi.nlm.nih.gov/gene?Db=gene\&Cmd=ShowDetailView\&TermToSearch=1116

133. Hakala BE, White C, Recklies AD. Human cartilage gp-39, a major secretory product of articular chondrocytes and synovial cells, is a mammalian member of a chitinase protein family. J Biol Chem. 1993;268(34):25803-25810. doi:10.1016/S0021-9258(19)74461-5

134. Rehli M, Krause SW, Andreesen R. Molecular Characterization of the Gene for Human Cartilage gp-39 (CHI3L1), a Member of the Chitinase Protein Family and Marker for Late Stages of Macrophage Differentiation. Genomics. 1997;43(2):221-225. doi:10.1006/GENO.1997.4778

135. CD44 CD44 molecule (Indian blood group) [ Homo sapiens (human) ]. https://www.ncbi.nlm.nih.gov/gene/960

136. TMEM219 transmembrane protein 219 [ Homo sapiens (human) ]. https://www.ncbi.nlm.nih.gov/gene?Db=gene\&Cmd=DetailsSearch\&Term=124446

137. Hao H, Chen H, Xie L, Liu H. YKL-40 promotes invasion and metastasis of bladder cancer by regulating epithelial mesenchymal transition. Ann Med. 2021;53(1):1169-1177. doi:10.1080/07853890.2021.1950920

138. Oh IH, Pyo JS, Son BK. Prognostic Impact of YKL-40 Immunohistochemical Expression in Patients with Colorectal Cancer. Curr Oncol 2021, Vol 28, Pages 3139-3149. 2021;28(4):3139-3149. doi:10.3390/CURRONCOL28040274

139. Chen A, Jiang Y, Li Z, et al. Chitinase-3-like 1 protein complexes modulate macrophage-mediated immune suppression in glioblastoma. J Clin Invest. 2021;131(16). doi:10.1172/JCI147552

140. Singh SK, Bhardwaj R, Wilczynska KM, Dumur CI, Kordula T. A complex of nuclear factor I-X3 and STAT3 regulates astrocyte and glioma migration through the secreted glycoprotein YKL-40. J Biol Chem. 2011;286(46):29893-29903. doi:10.1074/JBC.M111.257451

141. Francescone RA, Scully S, Faibish M, et al. Role of YKL-40 in the Angiogenesis, Radioresistance, and Progression of Glioblastoma. J Biol Chem. 2011;286(17):15332-15343. doi:10.1074/JBC.M110.212514

142. Bernardi D, Padoan A, Ballin A, et al. Serum YKL-40 following resection for cerebral glioblastoma. J Neurooncol. 2012;107(2):299-305. doi:10.1007/S11060-011-0762-7

143. Høgdall EVS, Ringsholt M, Høgdall CK, et al. YKL-40 tissue expression and plasma levels in patients with ovarian cancer. BMC Cancer. 2009;9(1):1-10. doi:10.1186/1471-2407-9-8/FIGURES/3

144. Momtazmanesh S, Shobeiri P, Saghazadeh A, et al. Neuronal and glial CSF biomarkers in multiple sclerosis: A systematic review and meta-analysis. Rev Neurosci. 2021;32(6):573-595. doi:10.1515/REVNEURO-2020-0145/ASSET/GRAPHIC/J_REVNEURO-2020-0145_FIG_007.JPG

145. Canto E, Tintore M, Villar LM, et al. Chitinase 3-like 1: prognostic biomarker in clinically isolated syndromes. Brain. 2015;138(Pt 4):918-931. doi:10.1093/BRAIN/AWV017

146. Tamam Y, Gunes B, Akbayir E, et al. CSF levels of HoxB3 and YKL-40 may predict conversion from clinically isolated syndrome to relapsing remitting multiple sclerosis. Mult Scler Relat Disord. 2021;48. doi:10.1016/J.MSARD.2020.102697

147. Comabella M, Fernández M, Martin R, et al. Cerebrospinal fluid chitinase 3-like 1 levels are associated with conversion to multiple sclerosis. Brain. 2010;133(Pt 4):1082-1093. doi:10.1093/BRAIN/AWQ035

148. De Fino C, Lucchini M, Lucchetti D, et al. The predictive value of CSF multiple assay in multiple sclerosis: A single center experience. Mult Scler Relat Disord. 2019;35:176-181. doi:10.1016/J.MSARD.2019.07.030 
149. Füvesi J, Hanrieder J, Bencsik K, et al. Proteomic analysis of cerebrospinal fluid in a fulminant case of multiple sclerosis. Int J Mol Sci. 2012;13(6):7676-7693. doi:10.3390/ijms13067676

150. Modvig S, Degn M, Roed H, et al. Cerebrospinal fluid levels of chitinase 3-like 1 and neurofilament light chain predict multiple sclerosis development and disability after optic neuritis. Mult Scler. 2015;21(14):1761-1770. doi:10.1177/1352458515574148

151. Martínez MAM, Olsson B, Bau L, et al. Glial and neuronal markers in cerebrospinal fluid predict progression in multiple sclerosis. Mult Scler J. 2015;21(5):550-561. doi:10.1177/1352458514549397

152. Novakova L, Axelsson M, Khademi M, et al. Cerebrospinal fluid biomarkers as a measure of disease activity and treatment efficacy in relapsing-remitting multiple sclerosis. J Neurochem. 2017;141(2):296-304. doi:10.1111/JNC.13881

153. Malmeström C, Axelsson M, Lycke J, Zetterberg H, Blennow K, Olsson B. CSF levels of YKL-40 are increased in MS and replaces with immunosuppressive treatment. J Neuroimmunol. 2014;269(1-2):87-89. doi:10.1016/J.JNEUROIM.2014.02.004

154. Burman J, Raininko R, Blennow K, Zetterberg H, Axelsson M, Malmeström C. YKL-40 is a CSF biomarker of intrathecal inflammation in secondary progressive multiple sclerosis. J Neuroimmunol. 2016;292:52-57. doi:10.1016/J.JNEUROIM.2016.01.013

155. Pérez-Miralles F, Prefasi D, García-Merino A, et al. CSF chitinase 3-like-1 association with disability of primary progressive MS. Neurol - Neuroimmunol Neuroinflammation. 2020;7(5). doi:10.1212/NXI.0000000000000815

156. Schneider R, Bellenberg B, Gisevius B, et al. Chitinase 3-like 1 and neurofilament light chain in CSF and CNS atrophy in MS. Neurol - Neuroimmunol Neuroinflammation. 2021;8(1). doi:10.1212/NXI.0000000000000906

157. Gil-Perotin S, Castillo-Villalba J, Cubas-Nuñez L, et al. Combined Cerebrospinal Fluid Neurofilament Light Chain Protein and Chitinase-3 Like-1 Levels in Defining Disease Course and Prognosis in Multiple Sclerosis. Front Neurol. 2019;10:1008. doi:10.3389/FNEUR.2019.01008/BIBTEX

158. Matute-Blanch C, Río J, Villar LM, et al. Chitinase 3-like 1 is associated with the response to interferonbeta treatment in multiple sclerosis. J Neuroimmunol. 2017;303:62-65. doi:10.1016/J.JNEUROIM.2016.12.006

159. Novakova L, Axelsson M, Khademi M, et al. Cerebrospinal fluid biomarkers of inflammation and degeneration as measures of fingolimod efficacy in multiple sclerosis. Mult Scler. 2017;23(1):62-71. doi:10.1177/1352458516639384

160. Møllgaard M, Degn M, Sellebjerg F, Frederiksen JL, Modvig S. Cerebrospinal fluid chitinase-3-like 2 and chitotriosidase are potential prognostic biomarkers in early multiple sclerosis. Eur J Neurol. 2016;23(5):898-905. doi:10.1111/ENE.12960

161. Comabella M, Sastre-Garriga J, Borras E, et al. CSF Chitinase 3-Like 2 Is Associated With Long-term Disability Progression in Patients With Progressive Multiple Sclerosis. Neurol - Neuroimmunol Neuroinflammation. 2021;8(6). doi:10.1212/NXI.0000000000001082

162. Aloisi F, Columba-Cabezas S, Franciotta D, et al. Lymphoid chemokines in chronic neuroinflammation. J Neuroimmunol. 2008;198(1-2):106-112. doi:10.1016/J.JNEUROIM.2008.04.025

163. Gunn MD, Ngo VN, Ansel KM, Ekland EH, Cyster JG, Williams LT. A B-cell-homing chemokine made in lymphoid follicles activates Burkitt's lymphoma receptor-1. Nat 1998 3916669. 1998;391(6669):799-803. doi:10.1038/35876

164. Londoño AC, Mora CA. Role of CXCL13 in the formation of the meningeal tertiary lymphoid organ in multiple sclerosis. F1000Research. 2018;7:514. doi:10.12688/F1000RESEARCH.14556.2

165. Magliozzi R, Howell O, Vora A, et al. Meningeal B-cell follicles in secondary progressive multiple sclerosis associate with early onset of disease and severe cortical pathology. Brain. 2007;130(4):1089-1104. doi:10.1093/BRAIN/AWM038 
166. Legler DF, Loetscher M, Roos RS, Clark-Lewis I, Baggiolini M, Moser B. B Cell-attracting Chemokine 1, a Human CXC Chemokine Expressed in Lymphoid Tissues, Selectively Attracts B Lymphocytes via BLR1/CXCR5. J Exp Med. 1998;187(4):655-660. doi:10.1084/JEM.187.4.655

167. Arneth BM. Impact of B cells to the pathophysiology of multiple sclerosis. J Neuroinflammation. 2019;16(1). doi:10.1186/S12974-019-1517-1

168. Ghafouri-Fard S, Honarmand K, Taheri M. A comprehensive review on the role of chemokines in the pathogenesis of multiple sclerosis. Metab Brain Dis. 2021;36(3):375-406. doi:10.1007/S11011-020-00648-6

169. Sellebjerg F, Börnsen L, Khademi M, et al. Increased cerebrospinal fluid concentrations of the chemokine CXCL13 in active MS. Neurology. 2009;73(23):2003-2010. doi:10.1212/WNL.0B013E3181C5B457

170. Alvarez E, Piccio L, Mikesell RJ, et al. CXCL13 is a biomarker of inflammation in multiple sclerosis, neuromyelitis optica, and other neurological conditions. Mult Scler J. 2013;19(9):1204-1208. doi:10.1177/1352458512473362

171. Khademi M, Kockum I, Andersson ML, et al. Cerebrospinal fluid CXCL13 in multiple sclerosis: a suggestive prognostic marker for the disease course. Mult Scler. 2011;17(3):335-343. doi:10.1177/1352458510389102

172. Kowarik MC, Cepok S, Sellner J, et al. CXCL13 is the major determinant for B cell recruitment to the CSF during neuroinflammation. J Neuroinflammation. 2012;9(1):1-11. doi:10.1186/1742-2094-9-93/TABLES/3

173. Krumbholz M, Theil D, Cepok S, et al. Chemokines in multiple sclerosis: CXCL12 and CXCL13 upregulation is differentially linked to CNS immune cell recruitment. Brain. 2006;129(1):200-211. doi:10.1093/BRAIN/AWH680

174. Ragheb S, Li Y, Simon K, et al. Multiple sclerosis: BAFF and CXCL13 in cerebrospinal fluid: http://dx.doi.org/101177/1352458511398887. 2011;17(7):819-829. doi:10.1177/1352458511398887

175. Kroksveen AC, Opsahl JA, Guldbrandsen A, et al. Cerebrospinal fluid proteomics in multiple sclerosis. Biochim Biophys Acta. 2015;1854(7):746-756. doi:10.1016/J.BBAPAP.2014.12.013

176. Ferraro D, Galli V, Vitetta F, et al. Cerebrospinal fluid CXCL13 in clinically isolated syndrome patients: Association with oligoclonal IgM bands and prediction of Multiple Sclerosis diagnosis. J Neuroimmunol. 2015;283:64-69. doi:10.1016/J.JNEUROIM.2015.04.011

177. Brettschneider J, Czerwoniak A, Senel M, et al. The Chemokine CXCL13 Is a Prognostic Marker in Clinically Isolated Syndrome (CIS). PLoS One. 2010;5(8):e11986. doi:10.1371/JOURNAL.PONE.0011986

178. Olesen MN, Soelberg K, Debrabant B, et al. Cerebrospinal fluid biomarkers for predicting development of multiple sclerosis in acute optic neuritis: A population-based prospective cohort study. $J$ Neuroinflammation. 2019;16(1):1-12. doi:10.1186/S12974-019-1440-5/FIGURES/6

179. Lindén M, Khademi M, Lima Bomfim I, et al. Multiple sclerosis risk genotypes correlate with an elevated cerebrospinal fluid level of the suggested prognostic marker CXCL13. Mult Scler. 2013;19(7):863-870. doi:10.1177/1352458512463482

180. Puthenparampil M, Federle L, Miante S, et al. BAFF Index and CXCL13 levels in the cerebrospinal fluid associate respectively with intrathecal IgG synthesis and cortical atrophy in multiple sclerosis at clinical onset. J Neuroinflammation. 2017;14(1):1-9. doi:10.1186/S12974-016-0785-2/FIGURES/4

181. DiSano KD, Gilli F, Pachner AR. Intrathecally produced CXCL13: A predictive biomarker in multiple sclerosis. Mult Scler J - Exp Transl Clin. 2020;6(4). doi:10.1177/2055217320981396

182. Lepennetier G, Hracsko Z, Unger M, et al. Cytokine and immune cell profiling in the cerebrospinal fluid of patients with neuro-inflammatory diseases. J Neuroinflammation. 2019;16(1):1-11. doi:10.1186/S12974019-1601-6/FIGURES/3

183. Sellebjerg F, Börnsen L, Ammitzbøll C, et al. Defining active progressive multiple sclerosis. Mult Scler. 2017;23(13):1727-1735. doi:10.1177/1352458517726592 
184. Iwanowski P, Losy J, Kramer L, Wójcicka M, Kaufman E. CXCL10 and CXCL13 chemokines in patients with relapsing remitting and primary progressive multiple sclerosis. J Neurol Sci. 2017;380:22-26. doi:10.1016/J.JNS.2017.06.048

185. Novakova L, Axelsson M, Malmeström C, et al. NFL and CXCL13 may reveal disease activity in clinically and radiologically stable MS. Mult Scler Relat Disord. 2020;46:102463. doi:10.1016/J.MSARD.2020.102463

186. Roach CA, Cross AH. Anti-CD20 B Cell Treatment for Relapsing Multiple Sclerosis. Front Neurol. $2021 ; 11$. doi:10.3389/FNEUR.2020.595547/PDF

187. Gelfand JM, Cree BAC, Hauser SL. Ocrelizumab and Other CD20 + B-Cell-Depleting Therapies in Multiple Sclerosis. Neurotherapeutics. 2017;14(4):835-841. doi:10.1007/S13311-017-0557-4

188. Riederer F. Ocrelizumab versus placebo in primary progressive multiple sclerosis. J fur Neurol Neurochir und Psychiatr. 2017;18(1):30-31.

doi:10.1056/NEJMOA1606468/SUPPL_FILE/NEJMOA1606468_DISCLOSURES.PDF

189. Bar-Or A, Calabresi PAJ, Arnlod D, et al. Rituximab in relapsing-remitting multiple sclerosis: a 72-week, open-label, phase I trial. Ann Neurol. 2008;63(3):395-400. doi:10.1002/ANA.21363

190. Naismith RT, Piccio L, Lyons JA, et al. Rituximab add-on therapy for breakthrough relapsing multiple sclerosis: a 52-week phase II trial. Neurology. 2010;74(23):1860-1867. doi:10.1212/WNL.0B013E3181E24373

191. McGinley MP, Goldschmidt CH, Rae-Grant AD. Diagnosis and Treatment of Multiple Sclerosis: A Review. JAMA. 2021;325(8):765-779. doi:10.1001/JAMA.2020.26858

192. Filippini G, Kruja J, Del Giovane C. Rituximab for people with multiple sclerosis. Cochrane database Syst Rev. 2021;11(11):CD013874. doi:10.1002/14651858.CD013874.PUB2

193. Piccio L, Naismith RT, Trinkaus K, et al. Changes in B- and T-lymphocyte and chemokine levels with rituximab treatment in multiple sclerosis. Arch Neurol. 2010;67(6):707-714.

doi:10.1001/ARCHNEUROL.2010.99

194. Cross AH, Klein RS, Piccio L. Rituximab combination therapy in relapsing multiple sclerosis. Ther Adv Neurol Disord. 2012;5(6):311-319. doi:10.1177/1756285612461165

195. Christensen JR, Ratzer R, Börnsen L, et al. Natalizumab in progressive MS. Neurology. 2014;82(17):14991507. doi:10.1212/WNL.0000000000000361

196. Romme Christensen J, Komori M, von Essen MR, et al. CSF inflammatory biomarkers responsive to treatment in progressive multiple sclerosis capture residual inflammation associated with axonal damage. Mult Scler J. 2019;25(7):937-946. doi:10.1177/1352458518774880

197. Axelsson M, Mattsson N, Malmeström C, Zetterberg H, Lycke J. The influence of disease duration, clinical course, and immunosuppressive therapy on the synthesis of intrathecal oligoclonal IgG bands in multiple sclerosis. J Neuroimmunol. 2013;264(1-2):100-105. doi:10.1016/J.JNEUROIM.2013.09.003

198. Karaaslan Z, Kurtuncu M, Akcay HI, et al. CXCL13 Levels Indicate Treatment Responsiveness to Fingolimod in MS Patients. Eur Neurol. 2022;85(1):69-71. doi:10.1159/000517770

199. Young MF, Kerr JM, Termine JD, et al. cDNA cloning, mRNA distribution and heterogeneity, chromosomal location, and RFLP analysis of human osteopontin (OPN). Genomics. 1990;7(4):491-502. doi:10.1016/0888-7543(90)90191-V

200. Gene: Spp1 (ENSMUSG00000029304) - Summary - Mus_musculus - Ensembl genome browser 105. Accessed February 15, 2022.

https:/www.ensembl.org/Mus_musculus/Gene/Summary?g=ENSMUSG00000029304;r=5:104582984104588916

201. SPP1 secreted phosphoprotein 1 [ Homo sapiens (human)]. https://www.ncbi.nlm.nih.gov/gene?cmd=retrieve\&dopt=default\&list_uids=6696\&rn=1

202. Bandopadhyay M, Bulbule A, Butti R, et al. Osteopontin as a therapeutic target for cancer. 
https://doi.org/101517/147282222014925447. 2014;18(8):883-895. doi:10.1517/14728222.2014.925447

203. Lund SA, Giachelli CM, Scatena M. The role of osteopontin in inflammatory processes. J Cell Commun Signal. 2009;3(3-4):311-322. doi:10.1007/S12079-009-0068-0/FIGURES/2

204. Agah E, Zardoui A, Saghazadeh A, Ahmadi M, Tafakhori A, Rezaei N. Osteopontin (OPN) as a CSF and blood biomarker for multiple sclerosis: A systematic review and meta-analysis. PLoS One. 2018;13(1):e0190252. doi:10.1371/JOURNAL.PONE.0190252

205. Cappellano G, Vecchio D, Magistrelli L, et al. The Yin-Yang of osteopontin in nervous system diseases: damage versus repair. Neural Regen Res. 2021;16(6):1131. doi:10.4103/1673-5374.300328

206. Boggio E, Dianzani C, Gigliotti CL, et al. Thrombin Cleavage of Osteopontin Modulates Its Activities in Human Cells In Vitro and Mouse Experimental Autoimmune Encephalomyelitis In Vivo. J Immunol Res. 2016;2016. doi:10.1155/2016/9345495

207. Wang J, Huang J, Zhu M, et al. Osteopontin potentiates PM-induced IL-1 $\alpha$ and IL-1 $\beta$ production via the ERK/JNK signaling pathway. Ecotoxicol Environ Saf. 2019;171:467-474. doi:10.1016/J.ECOENV.2019.01.005

208. Rittling SR, Singh R. Osteopontin in immune-mediated diseases. J Dent Res. 2015;94(12):1638-1645. doi:10.1177/0022034515605270

209. Castillo EF, Schluns KS. Regulating the immune system via IL-15 transpresentation. Cytokine. 2012;59(3):479-490. doi:10.1016/J.CYTO.2012.06.017

210. Han RK, Cheng YF, Zhou SS, et al. Increased circulating Th17 cell populations and elevated CSF osteopontin and IL-17 concentrations in patients with Guillain-Barré syndrome. J Clin Immunol. 2014;34(1):94-103. doi:10.1007/S10875-013-9965-3

211. Jones AP, Kermode AG, Lucas RM, Carroll WM, Nolan D, Hart PH. Circulating immune cells in multiple sclerosis. Clin Exp Immunol. 2017;187(2):193-203. doi:10.1111/CEI.12878

212. Jakovac H, Grubić Kezele T, Šućurović S, Mulac-Jeričević B, Radošević-Stašić B. Osteopontinmetallothionein I/II interactions in experimental autoimmune encephalomyelitis. Neuroscience. 2017;350:133-145. doi:10.1016/J.NEUROSCIENCE.2017.03.020

213. Chabas D, Baranzini SE, Mitchell D, et al. The influence of the proinflammatory cytokine, osteopontin, on autoimmue demyelinating desease. Science (80- ). 2001;294(5547):1731-1735. doi:10.1126/SCIENCE.1062960/SUPPL_FILE/1062960S1_THUMB.GIF

214. Chiocchetti A, Comi C, Indelicato M, et al. Osteopontin gene haplotypes correlate with multiple sclerosis development and progression. J Neuroimmunol. 2005;163(1-2):172-178.

doi:10.1016/J.JNEUROIM.2005.02.020

215. Comi C, Cappellano G, Chiocchetti A, et al. The impact of osteopontin gene variations on multiple sclerosis development and progression. Clin Dev Immunol. 2012;2012. doi:10.1155/2012/212893

216. Börnsen L, Khademi M, Olsson T, Sørensen PS, Sellebjerg F. Osteopontin concentrations are increased in cerebrospinal fluid during attacks of multiple sclerosis. Mult Scler. 2011;17(1):32-42. doi:10.1177/1352458510382247

217. Marastoni D, Magliozzi R, Bolzan A, et al. CSF Levels of CXCL12 and Osteopontin as Early Markers of Primary Progressive Multiple Sclerosis. Neurol Neuroimmunol neuroinflammation. 2021;8(6). doi:10.1212/NXI.0000000000001083

218. Orsi G, Cseh T, Hayden Z, et al. Microstructural and functional brain abnormalities in multiple sclerosis predicted by osteopontin and neurofilament light. Mult Scler Relat Disord. 2021;51. doi:10.1016/J.MSARD.2021.102923

219. Orsi G, Hayden Z, Cseh T, Berki T, Illes Z. Osteopontin levels are associated with late-time lower regional brain volumes in multiple sclerosis. Sci Reports 2021 111. 2021;11(1):1-7. doi:10.1038/s41598-021-03173-3

220. Jafarinia M, Sadeghi E, Alsahebfosoul F, Etemadifar M, Jahanbani-Ardakani H. Evaluation of plasma 
Osteopontin level in relapsing- remitting multiple sclerosis patients compared to healthy subjects in Isfahan Province. https://doi.org/101080/0020745420191694925. 2019;130(5):493-498. doi:10.1080/00207454.2019.1694925

221. Kivisäkk P, Healy BC, Francois K, et al. Evaluation of circulating osteopontin levels in an unselected cohort of patients with multiple sclerosis: Relevance for biomarker development. Mult Scler J. 2014;20(4):438-444. doi:10.1177/1352458513503052

222. Runia TF, Van Meurs M, Nasserinejad K, Hintzen RQ. No evidence for an association of osteopontin plasma levels with disease activity in multiple sclerosis. Mult Scler J. 2014;20(12):1670-1671. doi:10.1177/1352458514528765

223. Chowdhury SA, Lin J, Sadiq SA. Specificity and correlation with disease activity of cerebrospinal fluid osteopontin levels in patients with multiple sclerosis. Arch Neurol. 2008;65(2):232-235.

doi:10.1001/ARCHNEUROL.2007.33

224. Romme Christensen J, Börnsen L, Khademi M, et al. CSF inflammation and axonal damage are increased and correlate in progressive multiple sclerosis. Mult Scler. 2013;19(7):877-884.

doi:10.1177/1352458512466929

225. Wen SR, Liu GJ, Feng RN, et al. Increased levels of IL-23 and osteopontin in serum and cerebrospinal fluid of multiple sclerosis patients. J Neuroimmunol. 2012;244(1-2):94-96.

doi:10.1016/J.JNEUROIM.2011.12.004

226. Szalardy L, Zadori D, Simu M, Bencsik K, Vecsei L, Klivenyi P. Evaluating biomarkers of neuronal degeneration and neuroinflammation in CSF of patients with multiple sclerosis-osteopontin as a potential marker of clinical severity. J Neurol Sci. 2013;331(1-2):38-42. doi:10.1016/J.JNS.2013.04.024

227. Khademi M, Bornsen L, Rafatnia F, et al. The effects of natalizumab on inflammatory mediators in multiple sclerosis: prospects for treatment-sensitive biomarkers. Eur J Neurol. 2009;16(4):528-536. doi:10.1111/J.1468-1331.2009.02532.X

228. Iaffaldano P, Ruggieri M, Viterbo RG, Mastrapasqua M, Trojano M. The improvement of cognitive functions is associated with a decrease of plasma Osteopontin levels in Natalizumab treated relapsing multiple sclerosis. Brain Behav Immun. 2014;35:96-101. doi:10.1016/J.BBI.2013.08.009

229. Hong J, Hutton GJ. Regulatory Effects of Interferon- $\beta$ on Osteopontin and Interleukin-17 Expression in Multiple Sclerosis. https://home.liebertpub.com/jir. 2010;30(10):751-757. doi:10.1089/JIR.2010.0082

230. Yuan A, Rao M V., Veeranna, Nixon RA. Neurofilaments and Neurofilament Proteins in Health and Disease. Cold Spring Harb Perspect Biol. 2017;9(4). doi:10.1101/CSHPERSPECT.A018309

231. Schlaepfer WW, Lynch RG. Immunofluorescence studies of neurofilaments in the rat and human peripheral and central nervous system. J Cell Biol. 1977;74(1):241-250. doi:10.1083/JCB.74.1.241

232. Yuan A, Rao M V., Sasaki T, et al. $\alpha$-Internexin Is Structurally and Functionally Associated with the Neurofilament Triplet Proteins in the Mature CNS. J Neurosci. 2006;26(39):10006-10019. doi:10.1523/JNEUROSCI.2580-06.2006

233. Mücke N, Kämmerer L, Winheim S, et al. Assembly Kinetics of Vimentin Tetramers to Unit-Length Filaments: A Stopped-Flow Study. Biophys J. 2018;114(10):2408-2418. doi:10.1016/J.BPJ.2018.04.032

234. Yan Y, Brown A. Neurofilament Polymer Transport in Axons. J Neurosci. 2005;25(30):7014-7021. doi:10.1523/JNEUROSCI.2001-05.2005

235. Yuan A, Sasaki T, Rao M V., et al. Neurofilaments Form a Highly Stable Stationary Cytoskeleton after Reaching a Critical Level in Axons. J Neurosci. 2009;29(36):11316-11329. doi:10.1523/JNEUROSCI.194209.2009

236. Rao M V., Campbell J, Yuan A, et al. The neurofilament middle molecular mass subunit carboxylterminal tail domains is essential for the radial growth and cytoskeletal architecture of axons but not for regulating neurofilament transport rate. J Cell Biol. 2003;163(5):1021-1031. doi:10.1083/JCB.200308076 
237. Barry DM, Stevenson W, Bober BG, et al. Expansion of Neurofilament Medium C Terminus Increases Axonal Diameter Independent of Increases in Conduction Velocity or Myelin Thickness. J Neurosci. 2012;32(18):6209-6219. doi:10.1523/JNEUROSCI.0647-12.2012

238. Yuan A, Sershen H, Veeranna, et al. Functions of neurofilaments in synapses. Mol Psychiatry 2015208. 2015;20(8):915-915. doi:10.1038/mp.2015.99

239. Julien JP, Mushynski WE. Neurofilaments in health and disease. Prog Nucleic Acid Res Mol Biol. 1998;61:123. doi:10.1016/S0079-6603(08)60823-5

240. Stone EJ, Kolb SJ, Brown A. A review and analysis of the clinical literature on Charcot-Marie-Tooth disease caused by mutations in neurofilament protein L. Cytoskeleton. 2021;78(3):97-110. doi:10.1002/CM.21676

241. Khalil M, Teunissen CE, Otto M, et al. Neurofilaments as biomarkers in neurological disorders. Nat Rev Neurol 2018 1410. 2018;14(10):577-589. doi:10.1038/s41582-018-0058-z

242. Gaetani L, Blennow K, Calabresi P, Di Filippo M, Parnetti L, Zetterberg H. Neurofilament light chain as a biomarker in neurological disorders. J Neurol Neurosurg Psychiatry. 2019;90(8). doi:10.1136/JNNP-2018320106

243. Von Bartheld CS, Altick AL. Multivesicular bodies in neurons: distribution, protein content, and trafficking functions. Prog Neurobiol. 2011;93(3):313-340. doi:10.1016/J.PNEUROBIO.2011.01.003

244. Albargothy NJ, Johnston DA, MacGregor-Sharp M, et al. Convective influx/glymphatic system: tracers injected into the CSF enter and leave the brain along separate periarterial basement membrane pathways. Acta Neuropathol. 2018;136(1):139-152. doi:10.1007/S00401-018-1862-7

245. Kuhle J, Barro C, Andreasson U, et al. Comparison of three analytical platforms for quantification of the neurofilament light chain in blood samples: ELISA, electrochemiluminescence immunoassay and Simoa. Clin Chem Lab Med. 2016;54(10):1655-1661. doi:10.1515/CCLM-2015-1195

246. Alagaratnam J, Von Widekind S, De Francesco D, et al. Correlation between CSF and blood neurofilament light chain protein: a systematic review and meta-analysis. BMJ Neurol open. 2021;3(1).

doi:10.1136/BMJNO-2021-000143

247. Yuan A, Sasaki T, Kumar A, et al. Peripherin is a subunit of peripheral nerve neurofilaments: implications for differential vulnerability of CNS and peripheral nervous system axons. J Neurosci. 2012;32(25):85018508. doi:10.1523/JNEUROSCI.1081-12.2012

248. Uher T, McComb M, Galkin S, et al. Neurofilament levels are associated with blood-brain barrier integrity, lymphocyte extravasation, and risk factors following the first demyelinating event in multiple sclerosis. Mult Scler. 2021;27(2):220-231. doi:10.1177/1352458520912379

249. Mariotto S, Farinazzo A, Magliozzi R, Alberti D, Monaco S, Ferrari S. Serum and cerebrospinal neurofilament light chain levels in patients with acquired peripheral neuropathies. J Peripher Nerv Syst. 2018;23(3):174-177. doi:10.1111/JNS.12279

250. Körtvelyessy P, Kuhle J, Düzel E, et al. Ratio and index of Neurofilament light chain indicate its origin in Guillain-Barré Syndrome. Ann Clin Transl Neurol. 2020;7(11):2213-2220. doi:10.1002/ACN3.51207

251. Aeschbacher S, Kuhle J, Benkert P, et al. P2908Serum light-chain neurofilament, a brain lesion marker, correlates with CHA2DS2-VASc score among patients with atrial fibrillation: a cross-sectional study. Eur Heart J. 2018;39(suppl_1). doi:10.1093/EURHEARTJ/EHY565.P2908

252. Beydoun MA, Noren Hooten N, Maldonado AI, et al. BMI and Allostatic Load Are Directly Associated with Longitudinal Increase in Plasma Neurofilament Light among Urban Middle-Aged Adults. J Nutr. 2022;152(2):535-549. doi:10.1093/JN/NXAB381

253. Manouchehrinia A, Piehl F, Hillert J, et al. Confounding effect of blood volume and body mass index on blood neurofilament light chain levels. Ann Clin Transl Neurol. 2020;7(1):139-143. doi:10.1002/ACN3.50972

254. Sweeney MD, Sagare AP, Zlokovic B V. Blood-brain barrier breakdown in Alzheimer disease and other 
neurodegenerative disorders. Nat Rev Neurol. 2018;14(3):133-150. doi:10.1038/NRNEUROL.2017.188

255. Disanto G, Barro C, Benkert P, et al. Serum Neurofilament light: A biomarker of neuronal damage in multiple sclerosis. Ann Neurol. 2017;81(6):857-870. doi:10.1002/ANA.24954

256. Akamine S, Marutani N, Kanayama D, et al. Renal function is associated with blood neurofilament light chain level in older adults. Sci Reports 2020 101. 2020;10(1):1-7. doi:10.1038/s41598-020-76990-7

257. Koini M, Pirpamer L, Hofer E, et al. Factors influencing serum neurofilament light chain levels in normal aging. Aging (Albany NY). 2021;13(24):25729-25738. doi:10.18632/AGING.203790

258. Ladang A, Kovacs S, Lengelé L, et al. Neurofilament light chain concentration in an aging population. Aging Clin Exp Res. Published online 2022. doi:10.1007/S40520-021-02054-Z

259. Ashton NJ, Janelidze S, Al Khleifat A, et al. A multicentre validation study of the diagnostic value of plasma neurofilament light. Nat Commun. 2021;12(1). doi:10.1038/S41467-021-23620-Z

260. Hansson O, Janelidze S, Hall S, et al. Blood-based NfL: A biomarker for differential diagnosis of parkinsonian disorder. Neurology. 2017;88(10):930-937. doi:10.1212/WNL.0000000000003680

261. Benatar M, Zhang L, Wang L, et al. Validation of serum neurofilaments as prognostic and potential pharmacodynamic biomarkers for ALS. Neurology. 2020;95(1):59-69. doi:10.1212/WNL.0000000000009559

262. Guedes VA, Kenney K, Shahim P, et al. Exosomal neurofilament light: A prognostic biomarker for remote symptoms after mild traumatic brain injury? Neurology. 2020;94(23):e2412-e2423.

doi:10.1212/WNL.0000000000009577

263. Pekny M, Wilhelmsson U, Stokowska A, Tatlisumak T, Jood K, Pekna M. Neurofilament Light Chain (NfL) in Blood-A Biomarker Predicting Unfavourable Outcome in the Acute Phase and Improvement in the Late Phase after Stroke. Cells. 2021;10(6). doi:10.3390/CELLS10061537

264. Bäckström D, Linder J, Jakobson Mo S, et al. NfL as a biomarker for neurodegeneration and survival in Parkinson disease. Neurology. 2020;95(7):e827-e838. doi:10.1212/WNL.0000000000010084

265. Lycke JN, Karlsson JE, Andersen O, Rosengren LE. Neurofilament protein in cerebrospinal fluid: a potential marker of activity in multiple sclerosis. J Neurol Neurosurg Psychiatry. 1998;64(3):402-404. doi:10.1136/JNNP.64.3.402

266. Bartoš A, Fialová L, Soukupová J, Kukal J, Malbohan I, Pitha J. Antibodies against light neurofilaments in multiple sclerosis patients. Acta Neurol Scand. 2007;116(2):100-107. doi:10.1111/J.1600-0404.2006.00794.X

267. Thebault S, Reaume M, Marrie RA, et al. High or increasing serum NfL is predictive of impending multiple sclerosis relapses. Mult Scler Relat Disord. 2022;59:103535. doi:10.1016/J.MSARD.2022.103535

268. Salzer J, Svenningsson A, Sundström P. Neurofilament light as a prognostic marker in multiple sclerosis. Mult Scler. 2010;16(3):287-292. doi:10.1177/1352458509359725

269. Amor S, Van Der Star BJ, Bosca I, et al. Neurofilament light antibodies in serum reflect response to natalizumab treatment in multiple sclerosis. Mult Scler. 2014;20(10):1355-1362.

doi:10.1177/1352458514521887

270. Novakova L, Zetterberg H, Sundström P, et al. Monitoring disease activity in multiple sclerosis using serum neurofilament light protein. Neurology. 2017;89(22):2230-2237. doi:10.1212/WNL.0000000000004683

271. Damasceno A, Dias-Carneiro RPC, Moraes AS, et al. Clinical and MRI correlates of CSF neurofilament light chain levels in relapsing and progressive MS. Mult Scler Relat Disord. 2019;30:149-153. doi:10.1016/J.MSARD.2019.02.004

272. Wong YYM, Bruijstens AL, Barro C, et al. Serum neurofilament light chain in pediatric MS and other acquired demyelinating syndromes. Neurology. 2019;93(10):E968-E974. doi:10.1212/WNL.0000000000008057

273. Proschmann U, Haase R, Inojosa H, Akgün K, Ziemssen T. Drug and Neurofilament Levels in Serum and Breastmilk of Women With Multiple Sclerosis Exposed to Natalizumab During Pregnancy and Lactation. 
Front Immunol. 2021;12:3380. doi:10.3389/FIMMU.2021.715195/BIBTEX

274. Kuhle J, Daizadeh N, Benkert P, et al. Sustained reduction of serum neurofilament light chain over 7 years by alemtuzumab in early relapsing-remitting MS. Mult Scler. Published online 2021. doi:10.1177/13524585211032348

275. Dalla Costa G, Martinelli V, Sangalli F, et al. Prognostic value of serum neurofilaments in patients with clinically isolated syndromes. Neurology. 2019;92(7):E733-E741. doi:10.1212/WNL.0000000000006902

276. Thebault S, Tessier DR, Lee H, et al. High serum neurofilament light chain normalizes after hematopoietic stem cell transplantation for MS. Neurol - Neuroimmunol Neuroinflammation. 2019;6(5):598. doi:10.1212/NXI.0000000000000598

277. Sejbaek T, Nielsen HH, Penner N, et al. Dimethyl fumarate decreases neurofilament light chain in CSF and blood of treatment naïve relapsing MS patients. J Neurol Neurosurg Psychiatry. 2019;90(12):1324-1330. doi:10.1136/JNNP-2019-321321

278. Ehling R, Lutterotti A, Wanschitz J, et al. Increased frequencies of serum antibodies to neurofilament light in patients with primary chronic progressive multiple sclerosis. Mult Scler. 2004;10(6):601-606. doi:10.1191/1352458504MS1100OA

279. Håkansson I, Tisell A, Cassel P, et al. Neurofilament levels, disease activity and brain volume during follow-up in multiple sclerosis. J Neuroinflammation. 2018;15(1). doi:10.1186/S12974-018-1249-7

280. Lokhande H, Rosso M, Tauhid S, et al. Serum NfL levels in the first five years predict 10-year thalamic fraction in patients with MS. Mult Scler J - Exp Transl Clin. 2022;8(1). doi:10.1177/20552173211069348

281. Kuhle J, Plavina T, Barro C, et al. Neurofilament light levels are associated with long-term outcomes in multiple sclerosis. Mult Scler. 2020;26(13):1691-1699. doi:10.1177/1352458519885613

282. Verberk IMW, Koel-Simmelink M, Twaalfhoven H, et al. Ultrasensitive immunoassay allows measurement of serum neurofilament heavy in multiple sclerosis. Mult Scler Relat Disord. 2021;50. doi:10.1016/J.MSARD.2021.102840

283. Szilasiová J, Rosenberger J, Fedičová M, et al. Neurofilament Light Chain Levels Are Associated with Disease Activity Determined by No Evident Disease Activity in Multiple Sclerosis Patients. Eur Neurol. 2021;84(4):272-279. doi:10.1159/000515806

284. de Flon P, Laurell K, Sundström P, et al. Comparison of plasma and cerebrospinal fluid neurofilament light in a multiple sclerosis trial. Acta Neurol Scand. 2019;139(5):462-468. doi:10.1111/ANE.13078

285. Sehr T, Akgün K, Proschmann U, Bucki R, Zendzian-Piotrowska M, Ziemssen T. Early central vs. peripheral immunological and neurobiological effects of fingolimod-a longitudinal study. J Mol Med (Berl). 2019;97(9):1263-1271. doi:10.1007/S00109-019-01812-X

286. Fialová L., Bartos A., Soukupová J., Svarcová J., Ridzon P., Malbohan I. Synergy of serum and cerebrospinal fluid antibodies against axonal cytoskeletal proteins in patients with different neurological diseases. Folia Biol (Praha). 2009;55(1):23-26. Accessed February 15, 2022.

https://www.researchgate.net/publication/24430808_Synergy_of_serum_and_cerebrospinal_fluid_antibod ies_against_axonal_cytoskeletal_proteins_in_patients_with_different_neurological_diseases

287. Sejbaek T, Mendoza JP, Penner N, Madsen JS, Olsen DA, Illes Z. Comparison of neurofilament light chain results between two independent facilities. BMJ Neurol open. 2020;2(2). doi:10.1136/BMJNO-2020-000063

288. Niiranen M, Kontkanen A, Jääskeläinen O, et al. Serum GFAP and NfL levels in benign relapsingremitting multiple sclerosis. Mult Scler Relat Disord. 2021;56. doi:10.1016/J.MSARD.2021.103280

289. Kalatha T, Arnaoutoglou M, Koukoulidis T, et al. Does cognitive dysfunction correlate with neurofilament light polypeptide levels in the CSF of patients with multiple sclerosis? J Int Med Res. 2019;47(5):2187-2198. doi:10.1177/0300060519840550

290. Peng L, Bi C, Xia D, Mao L, Qian H. Increased cerebrospinal fluid neurofilament light chain in central nervous system inflammatory demyelinating disease. Mult Scler Relat Disord. 2019;30:123-128. 


\section{doi:10.1016/J.MSARD.2019.02.009}

291. Bridel C, Verberk IMW, Heijst JJA, Killestein J, Teunissen CE. Variations in consecutive serum neurofilament light levels in healthy controls and multiple sclerosis patients. Mult Scler Relat Disord. 2021;47. doi:10.1016/J.MSARD.2020.102666

292. Smets I, Holden D, Bianchi L, et al. Factors contributing to CSF NfL reduction over time in those starting treatment for multiple sclerosis: An observational study. Mult Scler Relat Disord. 2022;57. doi:10.1016/J.MSARD.2021.103409

293. Rosenstein I, Axelsson M, Novakova L, Blennow K, Zetterberg H, Lycke J. Exploring CSF neurofilament light as a biomarker for MS in clinical practice; a retrospective registry-based study. Mult Scler. Published online 2021. doi:10.1177/13524585211039104

294. Galetta K, Deshpande C, Healy BC, et al. Serum neurofilament levels and patient-reported outcomes in multiple sclerosis. Ann Clin Transl Neurol. 2021;8(3):631-638. doi:10.1002/acn3.51305

295. Bhan A, Jacobsen C, Myhr KM, Dalen I, Lode K, Farbu E. Neurofilaments and 10-year follow-up in multiple sclerosis. Mult Scler. 2018;24(10):1301-1307. doi:10.1177/1352458518782005

296. Reyes S, Smets I, Holden D, et al. CSF neurofilament light chain testing as an aid to determine treatment strategies in MS. Neurol Neuroimmunol neuroinflammation. 2020;7(6). doi:10.1212/NXI.0000000000000880

297. Zhang Y, Li X, Qiao J. Neurofilament protein light in multiple sclerosis. Chinese J Med. 2007;87(39):27452749. http://rs.yiigle.com/CN112137200739/117831.htm

298. Matute-Blanch C, Villar LM, Álvarez-Cermeño JC, et al. Neurofilament light chain and oligoclonal bands are prognostic biomarkers in radiologically isolated syndrome. Brain. 2018;141(4):1085-1093. doi:10.1093/BRAIN/AWY021

299. Pawlitzki M, Sweeney-Reed CM, Bittner D, et al. CSF-Progranulin and Neurofilament Light Chain Levels in Patients With Radiologically Isolated Syndrome-Sign of Inflammation. Front Neurol. 2018;9. doi:10.3389/FNEUR.2018.01075/PDF

300. Avsar T, Korkmaz D, Tütüncü M, et al. Protein biomarkers for multiple sclerosis: semi-quantitative analysis of cerebrospinal fluid candidate protein biomarkers in different forms of multiple sclerosis. Mult Scler. 2012;18(8):1081-1091. doi:10.1177/1352458511433303

301. van der Vuurst de Vries RM, Wong YYM, Mescheriakova JY, et al. High neurofilament levels are associated with clinically definite multiple sclerosis in children and adults with clinically isolated syndrome. Mult Scler. 2019;25(7):958-967. doi:10.1177/1352458518775303

302. Disanto G, Adiutori R, Dobson R, et al. Serum neurofilament light chain levels are increased in patients with a clinically isolated syndrome. J Neurol Neurosurg Psychiatry. 2016;87(2):126-129. doi:10.1136/jnnp2014-309690

303. Sellebjerg F, Royen L, Soelberg Sørensen P, Oturai AB, Jensen PEH. Prognostic value of cerebrospinal fluid neurofilament light chain and chitinase-3-like-1 in newly diagnosed patients with multiple sclerosis. Mult Scler. 2019;25(11):1444-1451. doi:10.1177/1352458518794308

304. Gaetani L, Eusebi P, Mancini A, et al. Cerebrospinal fluid neurofilament light chain predicts disease activity after the first demyelinating event suggestive of multiple sclerosis. Mult Scler Relat Disord. 2019;35:228-232. doi:10.1016/J.MSARD.2019.07.025

305. Bjornevik K, Munger KL, Cortese M, et al. Serum Neurofilament Light Chain Levels in Patients With Presymptomatic Multiple Sclerosis. JAMA Neurol. 2020;77(1):58-64. doi:10.1001/JAMANEUROL.2019.3238

306. Uphaus T, Steffen F, Muthuraman M, et al. NfL predicts relapse-free progression in a longitudinal multiple sclerosis cohort study. EBioMedicine. 2021;72. doi:10.1016/J.EBIOM.2021.103590

307. Thebault S, Abdoli M, Fereshtehnejad SM, Tessier D, Tabard-Cossa V, Freedman MS. Serum neurofilament light chain predicts long term clinical outcomes in multiple sclerosis. Sci Reports 2020101. 2020;10(1):1-11. doi:10.1038/s41598-020-67504-6 
308. Fissolo N, Pignolet B, Rio J, et al. Serum Neurofilament Levels and PML Risk in Patients With Multiple Sclerosis Treated With Natalizumab. Neurol Neuroimmunol neuroinflammation. 2021;8(4). doi:10.1212/NXI.0000000000001003

309. Toorop AA, van Lierop ZYG, Strijbis EEM, et al. Mild progressive multifocal leukoencephalopathy after switching from natalizumab to ocrelizumab. Neurol - Neuroimmunol Neuroinflammation. 2021;8(1). doi:10.1212/NXI.0000000000000904

310. Teunissen CE, Iacobaeus E, Khademi M, et al. Combination of CSF N-acetylaspartate and neurofilaments in multiple sclerosis. Neurology. 2009;72(15):1322-1329. doi:10.1212/WNL.0B013E3181A0FE3F

311. Cantó E, Barro C, Zhao C, et al. Association Between Serum Neurofilament Light Chain Levels and Longterm Disease Course Among Patients With Multiple Sclerosis Followed up for 12 Years. JAMA Neurol. 2019;76(11):1359-1366. doi:10.1001/JAMANEUROL.2019.2137

312. Larsson D, Åkerfeldt T, Carlson K, Burman J. Intrathecal immunoglobulins and neurofilament light after autologous haematopoietic stem cell transplantation for multiple sclerosis. Mult Scler. 2020;26(11):13511359. doi:10.1177/1352458519863983

313. Edwards KR, Garten L, Button J, O'Connor J, Kamath V, Frazier C. Neurofilament light chain as an indicator of exacerbation prior to clinical symptoms in multiple sclerosis. Mult Scler Relat Disord. 2019;31:59-61. doi:10.1016/J.MSARD.2019.03.016

314. Akgün K, Kretschmann N, Haase R, et al. Profiling individual clinical responses by high-frequency serum neurofilament assessment in MS. Neurol Neuroimmunol neuroinflammation. 2019;6(3). doi:10.1212/NXI.0000000000000555

315. Kuhle J, Nourbakhsh B, Grant D, et al. Serum neurofilament is associated with progression of brain atrophy and disability in early MS. Neurology. 2017;88(9):826-831. doi:10.1212/WNL.0000000000003653

316. Saraste M, Bezukladova S, Matilainen M, et al. High serum neurofilament associates with diffuse white matter damage in MS. Neurol Neuroimmunol neuroinflammation. 2020;8(1). doi:10.1212/NXI.0000000000000926

317. Anderson V, Bentley E, Loveless S, et al. Serum neurofilament-light concentration and real-world outcome in MS. J Neurol Sci. 2020;417. doi:10.1016/J.JNS.2020.117079

318. Ferraro D, Guicciardi C, De Biasi S, et al. Plasma neurofilaments correlate with disability in progressive multiple sclerosis patients. Acta Neurol Scand. 2020;141(1):16-21. doi:10.1111/ANE.13152

319. Holmøy T, Røsjø E, Zetterberg H, et al. Vitamin D supplementation and neurofilament light chain in multiple sclerosis. Acta Neurol Scand. 2019;139(2):172-176. doi:10.1111/ANE.13037

320. Hänninen K, Jääskeläinen O, Herukka SK, Soilu-Hänninen M. Vitamin D supplementation and serum neurofilament light chain in interferon-beta-1b-treated MS patients. Brain Behav. 2020;10(9).

doi:10.1002/BRB3.1772

321. Huss A, Senel M, Abdelhak A, et al. Longitudinal Serum Neurofilament Levels of Multiple Sclerosis Patients Before and After Treatment with First-Line Immunomodulatory Therapies. Biomedicines. 2020;8(9). doi:10.3390/BIOMEDICINES8090312

322. Quintana E, Coll C, Salavedra-Pont J, et al. Cognitive impairment in early stages of multiple sclerosis is associated with high cerebrospinal fluid levels of chitinase 3-like 1 and neurofilament light chain. Eur J Neurol. 2018;25(9):1189-1191. doi:10.1111/ENE.13687

323. Masvekar R, Phillips J, Komori M, Wu T, Bielekova B. Cerebrospinal Fluid Biomarkers of Myeloid and Glial Cell Activation Are Correlated With Multiple Sclerosis Lesional Inflammatory Activity. Front Neurosci. 2021;15. doi:10.3389/FNINS.2021.649876/PDF

324. Williams TE, Holdsworth KP, Nicholas JM, et al. Assessing Neurofilaments as Biomarkers of Neuroprotection in Progressive Multiple Sclerosis. Neurol - Neuroimmunol Neuroinflammation. 2022;9(2):e1130. doi:10.1212/NXI.0000000000001130 
325. Chitnis T, Gonzalez C, Healy BC, et al. Neurofilament light chain serum levels correlate with 10-year MRI outcomes in multiple sclerosis. Ann Clin Transl Neurol. 2018;5(12):1478-1491. doi:10.1002/ACN3.638

326. Virgilio E, Vecchio D, Crespi I, et al. Cerebrospinal fluid biomarkers and cognitive functions at multiple sclerosis diagnosis. J Neurol 2021. Published online January 28, 2022:1-9. doi:10.1007/S00415-021-10945-4

327. Sandberg L, Biström M, Salzer J, Vågberg M, Svenningsson A, Sundström P. Vitamin D and axonal injury in multiple sclerosis. Mult Scler. 2016;22(8):1027-1031. doi:10.1177/1352458515606986

328. Røsjø E, Lindstrøm JC, Holmøy T, Myhr KM, Varhaug KN, Torkildsen Ø. Natural Variation of Vitamin D and Neurofilament Light Chain in Relapsing-Remitting Multiple Sclerosis. Front Neurol. 2020;11. doi:10.3389/FNEUR.2020.00329/PDF

329. Proschmann U, Inojosa H, Akgün K, Ziemssen T. Natalizumab Pharmacokinetics and -Dynamics and Serum Neurofilament in Patients With Multiple Sclerosis. Front Neurol. 2021;12. doi:10.3389/FNEUR.2021.650530/PDF

330. Cruz-Gomez ÁJ, Forero L, Lozano-Soto E, et al. Cortical Thickness and Serum NfL Explain Cognitive Dysfunction in Newly Diagnosed Patients With Multiple Sclerosis. Neurol Neuroimmunol neuroinflammation. 2021;8(6). doi:10.1212/NXI.0000000000001074

331. Bhan A, Jacobsen C, Dalen I, et al. CSF neurofilament light chain predicts 10-year clinical and radiologic worsening in multiple sclerosis. Mult Scler J - Exp Transl Clin. 2021;7(4). doi:10.1177/20552173211060337

332. Khalil M, Pirpamer L, Hofer E, et al. Serum neurofilament light levels in normal aging and their association with morphologic brain changes. Nat Commun 2020 111. 2020;11(1):1-9. doi:10.1038/s41467020-14612-6

333. Gaetani L, Salvadori N, Lisetti V, et al. Cerebrospinal fluid neurofilament light chain tracks cognitive impairment in multiple sclerosis. J Neurol. 2019;266(9):2157-2163. doi:10.1007/S00415-019-09398-7

334. Friedova L, Motyl J, Srpova B, et al. The weak association between neurofilament levels at multiple sclerosis onset and cognitive performance after 9 years. Mult Scler Relat Disord. 2020;46. doi:10.1016/J.MSARD.2020.102534

335. Håkansson I, Johansson L, Dahle C, Vrethem M, Ernerudh J. Fatigue scores correlate with other selfassessment data, but not with clinical and biomarker parameters, in CIS and RRMS. Mult Scler Relat Disord. 2019;36. doi:10.1016/J.MSARD.2019.101424

336. Tauil CB, Rocha-Lima AD, Ferrari BB, et al. Depression and anxiety disorders in patients with multiple sclerosis: association with neurodegeneration and neurofilaments. Brazilian J Med Biol Res $=$ Rev Bras Pesqui medicas e Biol. 2021;54(3):1-6. doi:10.1590/1414-431X202010428

337. Højsgaard Chow H, Talbot J, Lundell H, et al. Dimethyl Fumarate Treatment in Patients With Primary Progressive Multiple Sclerosis: A Randomized, Controlled Trial. Neurol Neuroimmunol neuroinflammation. 2021;8(5). doi:10.1212/NXI.0000000000001037

338. Edwards KR, Kamath A, Button J, et al. A pharmacokinetic and biomarker study of delayed-release dimethyl fumarate in subjects with secondary progressive multiple sclerosis: evaluation of cerebrospinal fluid penetration and the effects on exploratory biomarkers. Mult Scler Relat Disord. 2021;51. doi:10.1016/J.MSARD.2021.102861

339. Mellergård J, Tisell A, Blystad I, et al. Cerebrospinal fluid levels of neurofilament and tau correlate with brain atrophy in natalizumab-treated multiple sclerosis. Eur J Neurol. 2017;24(1):112-121. doi:10.1111/ENE.13162

340. Kuhle J, Malmeström C, Axelsson M, et al. Neurofilament light and heavy subunits compared as therapeutic biomarkers in multiple sclerosis. Acta Neurol Scand. 2013;128(6). doi:10.1111/ANE.12151

341. Kapoor R, Sellebjerg F, Hartung H-P, et al. Natalizumab Reduces Serum Concentrations of Neurofilament Light Chain in Secondary Progressive Multiple Sclerosis Patients From the Phase 3 ASCEND Study (S12.008). Neurology. 2019;92(15 Supplement):S12.008.

http://n.neurology.org/content/92/15_Supplement/S12.008.abstract 
342. Dalla Costa G, Martinelli V, Moiola L, et al. Serum neurofilaments increase at progressive multifocal leukoencephalopathy onset in natalizumab-treated multiple sclerosis patients. Ann Neurol. 2019;85(4):606610. doi:10.1002/ANA.25437

343. Kuhle J, Disanto G, Lorscheider J, et al. Fingolimod and CSF neurofilament light chain levels in relapsingremitting multiple sclerosis. Neurology. 2015;84(16):1639-1643. doi:10.1212/WNL.0000000000001491

344. Piehl F, Kockum I, Khademi M, et al. Plasma neurofilament light chain levels in patients with MS switching from injectable therapies to fingolimod. Mult Scler. 2018;24(8):1046-1054. doi:10.1177/1352458517715132

345. Kuhle J, Kropshofer H, Häring DA, et al. Neurofilament light levels in the blood of patients with secondary progressive MS are higher than in primary progressive MS and may predict brain atrophy in both MS subtypes. Mult Scler J. 2018;24(Issue 2 Suppl.):111. doi:10.1177/1352458518798579

346. Allen-Philbey K, Trane S De, Mao Z, et al. Subcutaneous cladribine to treat multiple sclerosis: experience in 208 patients. Ther Adv Neurol Disord. 2021;14:17562864211057660. doi:10.1177/17562864211057661

347. Yildiz O, Mao Z, Adams A, et al. Disease activity in progressive multiple sclerosis can be effectively reduced by cladribine. Mult Scler Relat Disord. 2018;24:20-27. doi:10.1016/J.MSARD.2018.05.010

348. Delcoigne B, Manouchehrinia A, Barro C, et al. Blood neurofilament light levels segregate treatment effects in multiple sclerosis. Neurology. 2020;94(11):e1201-e1212. doi:10.1212/WNL.0000000000009097

349. Bar-Or A, Thanei G-A, Harp CT, et al. Blood neurofilament light levels are lowered to a healthy donor range in patients with RMS and PPMS following ocrelizumab treatment. Mult Scler J. 2019;25(Issue 2 Suppl):3-130. doi:10.1177/1352458519868070

350. Kuhle J, Kropshofer H, Barro C, et al. Siponimod Reduces Neurofilament Light Chain Blood Levels in Secondary Progressive Multiple Sclerosis Patients (S8.006). Neurology. 2018;90(15 Supplement):S8.006. http://n.neurology.org/content/90/15_Supplement/S8.006.abstract

351. Hauser SL, Bar-Or A, Cohen JA, et al. Ofatumumab versus Teriflunomide in Multiple Sclerosis. N Engl J Med. 2020;383(6):546-557. doi:10.1056/NEJMOA1917246

352. De Flon $\mathrm{P}$, Gunnarsson M, Laurell K, et al. Reduced inflammation in relapsing-remitting multiple sclerosis after therapy switch to rituximab. Neurology. 2016;87(2):141-147.

doi:10.1212/WNL.0000000000002832

353. Komori M, Kosa P, Stein J, et al. Pharmacodynamic effects of daclizumab in the intrathecal compartment. Ann Clin Transl Neurol. 2017;4(7):478-490. doi:10.1002/ACN3.427

354. Fox RJ, Raska P, Barro C, et al. Neurofilament light chain in a phase 2 clinical trial of ibudilast in progressive multiple sclerosis. Mult Scler. 2021;27(13):2014-2022. doi:10.1177/1352458520986956

355. Smolders J, Mimpen M, Oechtering J, et al. Vitamin D3 supplementation and neurofilament light chain in multiple sclerosis. Acta Neurol Scand. 2020;141(1):77-80. doi:10.1111/ANE.13185

356. Khalil M, Enzinger C, Langkammer C, et al. CSF neurofilament and N-acetylaspartate related brain changes in clinically isolated syndrome. Mult Scler. 2013;19(4):436-442. doi:10.1177/1352458512458010

357. Brettschneider J, Petzold A, Junker A, Tumani H. Axonal damage markers in the cerebrospinal fluid of patients with clinically isolated syndrome improve predicting conversion to definite multiple sclerosis. Mult Scler. 2006;12(2):143-148. doi:10.1191/135248506MS1263OA

358. Shehab AA, Solima DA, Abdel-Hafeez MA, Mohamed SM. Serum Phosphorylated Neurofilament Heavy Chain Level in Relapsing Remitting Multiple Sclerosis in Correlation to Disease Activity and Disability. Egypt J Immunol. 2019;26(1):1-13.

359. Herrera MI, Kölliker-Frers RA, Otero-Losada M, et al. A Pilot Cross-Sectional Study to Investigate the Biomarker Potential of Phosphorylated Neurofilament-H and Immune Mediators of Disability in Patients With 5 Year Relapsing-Remitting Multiple Sclerosis. Front Neurol. 2019;10.

doi:10.3389/FNEUR.2019.01046/PDF 
360. Williams T, Zetterberg H, Chataway J. Neurofilaments in progressive multiple sclerosis: a systematic review. J Neurol. 2021;268(9):3212-3222. doi:10.1007/S00415-020-09917-X

361. Petzold A, Tozer DJ, Schmierer K. Axonal damage in the making: neurofilament phosphorylation, proton mobility and magnetisation transfer in multiple sclerosis normal appearing white matter. Exp Neurol. 2011;232(2):234-239. doi:10.1016/J.EXPNEUROL.2011.09.011

362. Baulina NM, Kulakova OG, Favorova OO. MicroRNAs: The Role in Autoimmune Inflammation. Acta Naturae. 2016;8(1):21. doi:10.32607/20758251-2016-8-1-21-33

363. Friedman RC, Farh KKH, Burge CB, Bartel DP. Most mammalian mRNAs are conserved targets of microRNAs. Genome Res. 2009;19(1):92-105. doi:10.1101/GR.082701.108

364. Lewis BP, Burge CB, Bartel DP. Conserved seed pairing, often flanked by adenosines, indicates that thousands of human genes are microRNA targets. Cell. 2005;120(1):15-20. doi:10.1016/J.CELL.2004.12.035

365. Fromm B, Billipp T, Peck LE, et al. A Uniform System for the Annotation of Vertebrate microRNA Genes and the Evolution of the Human microRNAome. Annu Rev Genet. 2015;49:213-242. doi:10.1146/ANNUREV-GENET-120213-092023

366. Bartel DP. Metazoan MicroRNAs. Cell. 2018;173(1):20-51. doi:10.1016/J.CELL.2018.03.006

367. Rodriguez A, Griffiths-Jones S, Ashurst JL, Bradley A. Identification of mammalian microRNA host genes and transcription units. Genome Res. 2004;14(10A):1902-1910. doi:10.1101/GR.2722704

368. Kim YK, Kim VN. Processing of intronic microRNAs. EMBO J. 2007;26(3):775-783. doi:10.1038/SJ.EMBOJ.7601512

369. Baskerville S, Bartel DP. Microarray profiling of microRNAs reveals frequent coexpression with neighboring miRNAs and host genes. RNA. 2005;11(3):241-247. doi:10.1261/RNA.7240905

370. van den Berg MMJ, Krauskopf J, Ramaekers JG, Kleinjans JCS, Prickaerts J, Briedé JJ. Circulating microRNAs as potential biomarkers for psychiatric and neurodegenerative disorders. Prog Neurobiol. 2020;185. doi:10.1016/J.PNEUROBIO.2019.101732

371. Kosaka N, Yoshioka Y, Hagiwara K, Tominaga N, Katsuda T, Ochiya T. Trash or Treasure: extracellular microRNAs and cell-to-cell communication. Front Genet. 2013;4(SEP). doi:10.3389/FGENE.2013.00173

372. Mitchell PS, Parkin RK, Kroh EM, et al. Circulating microRNAs as stable blood-based markers for cancer detection. Proc Natl Acad Sci U S A. 2008;105(30):10513-10518. doi:10.1073/PNAS.0804549105

373. Pauley KM, Cha S, Chan EKL. MicroRNA in autoimmunity and autoimmune diseases. J Autoimmun. 2009;32(3-4):189-194. doi:10.1016/J.JAUT.2009.02.012

374. Kumar S, Reddy PH. Are circulating microRNAs peripheral biomarkers for Alzheimer's disease? Biochim Biophys Acta. 2016;1862(9):1617-1627. doi:10.1016/J.BBADIS.2016.06.001

375. Gandhi R. miRNA in multiple sclerosis: search for novel biomarkers. Mult Scler. 2015;21(9):1095-1103. doi:10.1177/1352458515578771

376. Junker A, Hohlfeld R, Meinl E. The emerging role of microRNAs in multiple sclerosis. Nat Rev Neurol. 2011;7(1):56-59. doi:10.1038/NRNEUROL.2010.179

377. Dolati S, Marofi F, Babaloo Z, et al. Dysregulated Network of miRNAs Involved in the Pathogenesis of Multiple Sclerosis. Biomed Pharmacother. 2018;104:280-290. doi:10.1016/J.BIOPHA.2018.05.050

378. Junker A, Krumbholz M, Eisele S, et al. MicroRNA profiling of multiple sclerosis lesions identifies modulators of the regulatory protein CD47. Brain. 2009;132(Pt 12):3342-3352. doi:10.1093/BRAIN/AWP300

379. Noorbakhsh F, Ellestad KK, Maingat F, et al. Impaired neurosteroid synthesis in multiple sclerosis. Brain. 2011;134(9):2703. doi:10.1093/BRAIN/AWR200

380. Tripathi A, Volsko C, Datta U, Regev K, Dutta R. Expression of disease-related miRNAs in white-matter lesions of progressive multiple sclerosis brains. Ann Clin Transl Neurol. 2019;6(5):854-862. 
doi:10.1002/ACN3.750

381. Fritsche L, Teuber-Hanselmann S, Soub D, Harnisch K, Mairinger F, Junker A. MicroRNA profiles of MS gray matter lesions identify modulators of the synaptic protein synaptotagmin-7. Brain Pathol. 2020;30(3):524-540. doi:10.1111/BPA.12800

382. Teuber-Hanselmann S, Meinl E, Junker A. MicroRNAs in gray and white matter multiple sclerosis lesions: impact on pathophysiology. J Pathol. 2020;250(5):496-509. doi:10.1002/PATH.5399

383. Wang H, Moyano AL, Ma Z, et al. miR-219 Cooperates with miR-338 in Myelination and Promotes Myelin Repair in the CNS. Dev Cell. 2017;40(6):566-582.e5. doi:10.1016/J.DEVCEL.2017.03.001

384. Du C, Liu C, Kang J, et al. MicroRNA miR-326 regulates TH-17 differentiation and is associated with the pathogenesis of multiple sclerosis. Nat Immunol. 2009;10(12):1252-1259. doi:10.1038/NI.1798

385. Baulina N, Kulakova O, Kiselev I, et al. Immune-related miRNA expression patterns in peripheral blood mononuclear cells differ in multiple sclerosis relapse and remission. J Neuroimmunol. 2018;317:67-76. doi:10.1016/J.JNEUROIM.2018.01.005

386. Zailaie SA, Siddiqui JJ, Al Saadi RM, Anbari DM, S. Alomari A, Cupler EJ. Serum Based miRNA as a Diagnostic Biomarker for Multiple Sclerosis: a Systematic Review and Meta-Analysis. Immunol Invest. Published online March 4, 2021:1-16. doi:10.1080/08820139.2021.1887888

387. Tai X, Van Laethem F, Pobezinsky L, et al. Basis of CTLA-4 function in regulatory and conventional CD4(+) T cells. Blood. 2012;119(22):5155-5163. doi:10.1182/blood-2011-11-388918

388. Viglietta V, Baecher-Allan C, Weiner HL, Hafler DA. Loss of functional suppression by CD4+CD25+ regulatory T cells in patients with multiple sclerosis. J Exp Med. 2004;199(7):971-979. doi:10.1084/JEM.20031579

389. Vistbakka J, Elovaara I, Lehtimäki T, Hagman S. Circulating microRNAs as biomarkers in progressive multiple sclerosis. Mult Scler. 2017;23(3):403-412. doi:10.1177/1352458516651141

390. M G-A, Y L, WH M, D P, MK R, AE L-R. Analysis of miRNA in Normal Appearing White Matter to Identify Altered CNS Pathways in Multiple Sclerosis. J autoimmune Disord. 2015;1(1). doi:10.21767/24718153.100006

391. Braccioli L, Vervoort SJ, Puma G, Nijboer CH, Coffer PJ. SOX4 inhibits oligodendrocyte differentiation of embryonic neural stem cells in vitro by inducing Hes5 expression. Stem Cell Res. 2018;33:110-119. doi:10.1016/J.SCR.2018.10.005

392. Rhead B, Shao X, Graves JS, et al. miRNA contributions to pediatric-onset multiple sclerosis inferred from GWAS. Ann Clin Transl Neurol. 2019;6(6):1053-1061. doi:10.1002/ACN3.786

393. Boziki MK, Kesidou E, Theotokis P, et al. Microbiome in Multiple Sclerosis; Where Are We, What We Know and Do Not Know. Brain Sci. 2020;10(4). doi:10.3390/BRAINSCI10040234

394. Maglione A, Zuccalà M, Tosi M, Clerico M, Rolla S. Host Genetics and Gut Microbiome: Perspectives for Multiple Sclerosis. Genes (Basel). 2021;12(8). doi:10.3390/GENES12081181

395. Kirby TO, Ochoa-Repáraz J. The Gut Microbiome in Multiple Sclerosis: A Potential Therapeutic Avenue. Med Sci 2018, Vol 6, Page 69. 2018;6(3):69. doi:10.3390/MEDSCI6030069

396. Nuzziello N, Vilardo L, Pelucchi P, et al. Investigating the Role of MicroRNA and Transcription Factor Co-regulatory Networks in Multiple Sclerosis Pathogenesis. Int J Mol Sci. 2018;19(11). doi:10.3390/IJMS19113652

397. Liguori M, Nuzziello N, Licciulli F, et al. Combined microRNA and mRNA expression analysis in pediatric multiple sclerosis: an integrated approach to uncover novel pathogenic mechanisms of the disease. Hum Mol Genet. 2018;27(1):66-79. doi:10.1093/HMG/DDX385

398. Wang AL, Rao VR, Chen JJ, et al. Role of FAM18B in diabetic retinopathy. Mol Vis. 2014;20:1146. Accessed February 15, 2022. /pmc/articles/PMC4124103/ 
399. Luo D, Wang J, Zhang X, Rang X, Xu C, Fu J. Identification and functional analysis of specific MS risk miRNAs and their target genes. Mult Scler Relat Disord. 2020;41. doi:10.1016/J.MSARD.2020.102044

400. Gandhi R, Healy B, Gholipour T, et al. Circulating microRNAs as biomarkers for disease staging in multiple sclerosis. Ann Neurol. 2013;73(6):729-740. doi:10.1002/ANA.23880

401. Ridolfi E, Fenoglio C, Cantoni C, et al. Expression and Genetic Analysis of MicroRNAs Involved in Multiple Sclerosis. Int J Mol Sci 2013, Vol 14, Pages 4375-4384. 2013;14(3):4375-4384.

doi:10.3390/IJMS14034375

402. Søndergaard HB, Hesse D, Krakauer M, Sørensen PS, Sellebjerg F. Differential microRNA expression in blood in multiple sclerosis. Mult Scler. 2013;19(14):1849-1857. doi:10.1177/1352458513490542

403. Kacperska MJ, Jastrzebski K, Tomasik B, Walenczak J, Konarska-Krol M, Glabinski A. Selected extracellular microRNA as potential biomarkers of multiple sclerosis activity--preliminary study. J Mol Neurosci. 2015;56(1):154-163. doi:10.1007/S12031-014-0476-3

404. Quintana E, Ortega FJ, Robles-Cedeño R, et al. miRNAs in cerebrospinal fluid identify patients with MS and specifically those with lipid-specific oligoclonal IgM bands. Mult Scler. 2017;23(13):1716-1726. doi:10.1177/1352458516684213

405. Regev K, Healy BC, Paul A, et al. Identification of MS-specific serum miRNAs in an international multicenter study. Neurol - Neuroimmunol Neuroinflammation. 2018;5(5):491.

doi:10.1212/NXI.0000000000000491

406. Munõz-San Martín M, Reverter G, Robles-Cedenõ R, et al. Analysis of miRNA signatures in CSF identifies upregulation of miR-21 and miR-146a/b in patients with multiple sclerosis and active lesions. J Neuroinflammation. 2019;16(1). doi:10.1186/S12974-019-1590-5

407. Piket E, Zheleznyakova GY, Kular L, Jagodic M. Small non-coding RNAs as important players, biomarkers and therapeutic targets in multiple sclerosis: A comprehensive overview. J Autoimmun. 2019;101:17-25. doi:10.1016/J.JAUT.2019.04.002

408. Mycko MP, Baranzini SE. microRNA and exosome profiling in multiple sclerosis. Mult Scler. 2020;26(5):599-604. doi:10.1177/1352458519879303

409. Walsh AD, Nguyen LT, Binder MD. miRNAs in Microglia: Important Players in Multiple Sclerosis Pathology. ASN Neuro. 2021;13. doi:10.1177/1759091420981182

410. Regev K, Healy BC, Khalid F, et al. Association Between Serum MicroRNAs and Magnetic Resonance Imaging Measures of Multiple Sclerosis Severity. JAMA Neurol. 2017;74(3):275-285.

doi:10.1001/JAMANEUROL.2016.5197

411. Hemond CC, Healy BC, Tauhid S, et al. MRI phenotypes in MS: Longitudinal changes and miRNA signatures. Neurol Neuroimmunol neuroinflammation. 2019;6(2). doi:10.1212/NXI.0000000000000530

412. Aslani S, Jafari N, Javan MR, Karami J, Ahmadi M, Jafarnejad M. Epigenetic Modifications and Therapy in Multiple Sclerosis. Neuromolecular Med. 2017;19(1):11-23. doi:10.1007/S12017-016-8422-X

413. Sanger HL, KLOTZt G, RIESNERt D, et al. Viroids are single-stranded covalently closed circular RNA molecules existing as highly base-paired rod-like structures. Proc Natl Acad Sci. 1976;73(11):3852-3856. doi:10.1073/PNAS.73.11.3852

414. Muñoz-Culla M, Irizar H, Otaegui D. The genetics of multiple sclerosis: review of current and emerging candidates. Appl Clin Genet. 2013;6:63-73. doi:10.2147/TACG.S29107

415. Küçükali Cİ, Kürtüncü M, Çoban A, Çebi M, Tüzün E. Epigenetics of multiple sclerosis: an updated review. Neuromolecular Med. 2015;17(2):83-96. doi:10.1007/S12017-014-8298-6

416. Selmaj I, Cichalewska M, Namiecinska M, et al. Global exosome transcriptome profiling reveals biomarkers for multiple sclerosis. Ann Neurol. 2017;81(5):703-717. doi:10.1002/ANA.24931

417. Zeng L, Cui J, Wu H, Lu Q. The emerging role of circulating microRNAs as biomarkers in autoimmune 
diseases. Autoimmunity. 2014;47(7):419-429. doi:10.3109/08916934.2014.929667

418. Cocquerelle C, Mascrez B, Hétuin D, Bailleul B. Mis-splicing yields circular RNA molecules. FASEB J Off Publ Fed Am Soc Exp Biol. 1993;7(1):155-160. doi:10.1096/fasebj.7.1.7678559

419. Moldovan LI, Hansen TB, Venø MT, et al. High-throughput RNA sequencing from paired lesional- and non-lesional skin reveals major alterations in the psoriasis circRNAome. BMC Med Genomics. 2019;12(1). doi:10.1186/S12920-019-0616-2

420. Jeck WR, Sharpless NE. Detecting and characterizing circular RNAs. Nat Biotechnol. 2014;32(5):453-461. doi:10.1038/NBT.2890

421. Wilusz JE. Repetitive elements regulate circular RNA biogenesis. Mob Genet Elements. 2015;5(3). doi:10.1080/2159256X.2015.1045682

422. Dragomir M, Calin GA. Circular RNAs in Cancer - Lessons Learned From microRNAs. Front Oncol. 2018;8(MAY). doi:10.3389/FONC.2018.00179

423. Zhang Y, Zhang XO, Chen T, et al. Circular intronic long noncoding RNAs. Mol Cell. 2013;51(6):792-806. doi:10.1016/J.MOLCEL.2013.08.017

424. Zhang ZC, Guo XL, Li X. The novel roles of circular RNAs in metabolic organs. Genes Dis. 2017;5(1):16-23. doi:10.1016/J.GENDIS.2017.12.002

425. Hansen TB, Jensen TI, Clausen BH, et al. Natural RNA circles function as efficient microRNA sponges. Nature. 2013;495(7441):384-388. doi:10.1038/NATURE11993

426. Hansen TB, Kjems J, Damgaard CK. Circular RNA and miR-7 in cancer. Cancer Res. 2013;73(18):5609-5612. doi:10.1158/0008-5472.CAN-13-1568

427. Tay Y, Rinn J, Pandolfi PP. The multilayered complexity of ceRNA crosstalk and competition. Nature. 2014;505(7483):344-352. doi:10.1038/NATURE12986

428. Bachmayr-Heyda A, Reiner AT, Auer K, et al. Correlation of circular RNA abundance with proliferation-exemplified with colorectal and ovarian cancer, idiopathic lung fibrosis, and normal human tissues. Sci Rep. 2015;5:8057. doi:10.1038/srep08057

429. Rybak-Wolf A, Stottmeister C, Glažar P, et al. Circular RNAs in the Mammalian Brain Are Highly Abundant, Conserved, and Dynamically Expressed. Mol Cell. 2015;58(5):870-885.

doi:10.1016/J.MOLCEL.2015.03.027

430. Cadena C, Hur S. Antiviral Immunity and Circular RNA: No End in Sight. Mol Cell. 2017;67(2):163-164. doi:10.1016/J.MOLCEL.2017.07.005

431. Yang L, Fu J, Zhou Y. Circular RNAs and Their Emerging Roles in Immune Regulation. Front Immunol. 2018;9. doi:10.3389/FIMMU.2018.02977/PDF

432. Zhang Y, Zhang Y, Li X, Zhang M, Lv K. Microarray analysis of circular RNA expression patterns in polarized macrophages. Int J Mol Med. 2017;39(2):373-379. doi:10.3892/IJMM.2017.2852

433. Iparraguirre L, Muñoz-Culla M, Prada-Luengo I, Castillo-Triviño T, Olascoaga J, Otaegui D. Circular RNA profiling reveals that circular RNAs from ANXA2 can be used as new biomarkers for multiple sclerosis. Hum Mol Genet. 2017;26(18):3564-3572. doi:10.1093/HMG/DDX243

434. Cardamone G, Paraboschi EM, Rimoldi V, Duga S, Soldà G, Asselta R. The Characterization of GSDMB Splicing and Backsplicing Profiles Identifies Novel Isoforms and a Circular RNA That Are Dysregulated in Multiple Sclerosis. Int J Mol Sci 2017, Vol 18, Page 576. 2017;18(3):576. doi:10.3390/IJMS18030576

435. Cardamone G, Paraboschi EM, Soldà G, et al. Not only cancer: the long non-coding RNA MALAT1 affects the repertoire of alternatively spliced transcripts and circular RNAs in multiple sclerosis. Hum Mol Genet. 2019;28(9):1414-1428. doi:10.1093/HMG/DDY438

436. Iparraguirre L, Alberro A, Sepúlveda L, et al. RNA-Seq profiling of leukocytes reveals a sex-dependent global circular RNA upregulation in multiple sclerosis and 6 candidate biomarkers. Hum Mol Genet. 


\section{0;29(20):3361-3372. doi:10.1093/HMG/DDAA219}

437. Muñoz-Culla M, Irizar H, Sáenz-Cuesta M, et al. SncRNA (microRNA \&snoRNA) opposite expression pattern found in multiple sclerosis relapse and remission is sex dependent. Sci Rep. 2016;6. doi:10.1038/SREP20126

438. Gregory SG, Schmidt S, Seth P, et al. Interleukin 7 receptor alpha chain (IL7R) shows allelic and functional association with multiple sclerosis. Nat Genet. 2007;39(9):1083-1091. doi:10.1038/NG2103

439. Lundmark F, Duvefelt K, Iacobaeus E, et al. Variation in interleukin 7 receptor alpha chain (IL7R) influences risk of multiple sclerosis. Nat Genet. 2007;39(9):1108-1113. doi:10.1038/NG2106

440. Tavakolpour S. Interleukin 7 receptor polymorphisms and the risk of multiple sclerosis: A meta-analysis. Mult Scler Relat Disord. 2016;8:66-73. doi:10.1016/J.MSARD.2016.05.001

441. Traboulsee AL, Bernales CQ, Ross JP, Lee JD, Sadovnick AD, Vilariño-Güell C. Genetic variants in IL2RA and IL7R affect multiple sclerosis disease risk and progression. Neurogenetics. 2014;15(3):165-169. doi:10.1007/S10048-014-0403-3

442. Eftekharian MM, Noroozi R, Komaki A, Mazdeh M, Ghafouri-Fard S, Taheri M. MALAT1 Genomic Variants and Risk of Multiple Sclerosis. Immunol Invest. 2019;48(5):549-554.

doi:10.1080/08820139.2019.1576728

443. Masoumi F, Ghorbani S, Talebi F, et al. Malat1 long noncoding RNA regulates inflammation and leukocyte differentiation in experimental autoimmune encephalomyelitis. J Neuroimmunol. 2019;328:50-59. doi:10.1016/J.JNEUROIM.2018.11.013

444. Shaker OG, Mahmoud RH, Abdelaleem OO, et al. LncRNAs, MALAT1 and lnc-DC as potential biomarkers for multiple sclerosis diagnosis. Biosci Rep. 2019;39(1). doi:10.1042/BSR20181335

445. Paraboschi EM, Cardamone G, Soldà G, Duga S, Asselta R. Interpreting Non-coding Genetic Variation in Multiple Sclerosis Genome-Wide Associated Regions. Front Genet. 2018;9. doi:10.3389/FGENE.2018.00647/PDF

446. Żurawska A, Mycko MP, Selmaj KW. Dominant role of circular RNA in miRNA circuit in multiple sclerosis. Mult Scler J. Published online 2018:530-737. doi:https://doi.org/10.1177/1352458518798591

447. Tezuka K, Suzuki M, Sato R, Kawarada S, Terasaki T, Uchida Y. Activation of Annexin A2 signaling at the blood-brain barrier in a mouse model of multiple sclerosis. J Neurochem. Published online February 5, 2022. doi:10.1111/JNC.15578

448. Zurawska AE, Mycko MP, Selmaj I, Raine CS, Selmaj KW. Multiple Sclerosis. Neurol - Neuroimmunol Neuroinflammation. 2021;8(5). doi:10.1212/NXI.0000000000001041

449. Iparraguirre L, Alberro A, Hansen TB, Castillo-Triviño T, Muñoz-Culla M, Otaegui D. Profiling of Plasma Extracellular Vesicle Transcriptome Reveals That circRNAs Are Prevalent and Differ between Multiple Sclerosis Patients and Healthy Controls. Biomedicines. 2021;9(12). doi:10.3390/BIOMEDICINES9121850

450. Iparraguirre L, Olaverri D, Blasco T, et al. Whole-Transcriptome Analysis in Peripheral Blood Mononuclear Cells from Patients with Lipid-Specific Oligoclonal IgM Band Characterization Reveals Two Circular RNAs and Two Linear RNAs as Biomarkers of Highly Active Disease. Biomedicines. 2020;8(12):116. doi:10.3390/BIOMEDICINES8120540

451. Moreno-García L, López-Royo T, Calvo AC, et al. Competing Endogenous RNA Networks as Biomarkers in Neurodegenerative Diseases. Int J Mol Sci. 2020;21(24):1-42. doi:10.3390/IJMS21249582

452. Lodde V, Murgia G, Simula ER, Steri M, Floris M, Idda ML. Long Noncoding RNAs and Circular RNAs in Autoimmune Diseases. Biomolecules. 2020;10(7):1-22. doi:10.3390/BIOM10071044

453. Ghafouri-Fard S, Taheri M. A comprehensive review of non-coding RNAs functions in multiple sclerosis. Eur J Pharmacol. 2020;879. doi:10.1016/J.EJPHAR.2020.173127

454. Chen M, Lai X, Wang X, et al. Long Non-coding RNAs and Circular RNAs: Insights Into Microglia and 
Astrocyte Mediated Neurological Diseases. Front Mol Neurosci. 2021;14. doi:10.3389/FNMOL.2021.745066/PDF

455. Friese MA, Schattling B, Fugger L. Mechanisms of neurodegeneration and axonal dysfunction in multiple sclerosis. Nat Rev Neurol. 2014;10(4):225-238. doi:10.1038/NRNEUROL.2014.37

456. Montalban X, Gold R, Thompson AJ, et al. ECTRIMS/EAN guideline on the pharmacological treatment of people with multiple sclerosis. Eur J Neurol. 2018;25(2):215-237. doi:10.1111/ENE.13536

457. Sima DM, Esposito G, Van Hecke W, Ribbens A, Nagels G, Smeets D. Health Economic Impact of Software-Assisted Brain MRI on Therapeutic Decision-Making and Outcomes of Relapsing-Remitting Multiple Sclerosis Patients\&mdash;A Microsimulation Study. Brain Sci 2021, Vol 11, Page 1570. 2021;11(12):1570. doi:10.3390/BRAINSCI11121570

458. Monschein T, Salhofer-Polanyi S, Altmann P, et al. Should I stop or should I go on? Disease modifying therapy after the first clinical episode of multiple sclerosis. J Neurol. 2021;268(4):1247-1253. doi:10.1007/S00415-020-10074-4

459. Temmerman J, Van Der Veken F, Engelborghs S, et al. Brain Volume Loss Can Occur at the Rate of Normal Aging in Patients with Multiple Sclerosis Who Are Free from Disease Activity. J Clin Med 2022, Vol 11, Page 523. 2022;11(3):523. doi:10.3390/JCM11030523

460. Szilasiova J, Mikula P, Rosenberger J, et al. Associations between neurofilament light chain levels, disease activity and brain atrophy in progressive multiple sclerosis. Biomed Pap Med Fac Univ Palacky Olomouc Czech Repub. Published online June 1, 2021. doi:10.5507/BP.2021.034

461. Ziemssen T, Derfuss T, de Stefano N, et al. Optimizing treatment success in multiple sclerosis. J Neurol. 2016;263(6):1053-1065. doi:10.1007/S00415-015-7986-Y

462. Giovannoni G, Turner B, Gnanapavan S, Offiah C, Schmierer K, Marta M. Is it time to target no evident disease activity (NEDA) in multiple sclerosis? Mult Scler Relat Disord. 2015;4(4):329-333. doi:10.1016/J.MSARD.2015.04.006

463. Pandit L. No Evidence of Disease Activity (NEDA) in Multiple Sclerosis - Shifting the Goal Posts. Ann Indian Acad Neurol. 2019;22(3):261-263. doi:10.4103/AIAN.AIAN_159_19

464. Biernacki T, Sandi D, Kincses ZT, et al. Contributing factors to health-related quality of life in multiple sclerosis. Brain Behav. 2019;9(12). doi:10.1002/brb3.1466

465. Sandi D, Biernacki T, Szekeres D, et al. Prevalence of cognitive impairment among Hungarian patients with relapsing-remitting multiple sclerosis and clinically isolated syndrome. Mult Scler Relat Disord. 2017;17:57-62. doi:10.1016/J.MSARD.2017.06.017

466. Faragó P, Tóth E, Szabó N N, et al. Connection between microstructural alterations detected by diffusion MRI and cognitive dysfunction in MS: A model-free analysis approach. Mult Scler Relat Disord. 2022;57. doi:10.1016/J.MSARD.2021.103442

467. Tóth E, Faragó P, Király A, et al. The contribution of various MRI parameters to clinical and cognitive disability in multiple sclerosis. Front Neurol. 2019;10(JAN). doi:10.3389/FNEUR.2018.01172/PDF

468. Veréb D, Tóth E, Bozsik B, et al. Altered brain network function during attention-modulated visual processing in multiple sclerosis. Mult Scler. 2021;27(9):1341-1349. doi:10.1177/1352458520958360 